\title{
Intersection Numbers of Curves on Hilbert Modular Surfaces and Modular Forms of Nebentypus
}

F. Hirzebruch (Bonn) and D. Zagier (Bonn)*

To Jean-Pierre Serre

\section{Contents}

Introduction . . . . . . . . . . . . . . . . . . . . . . . . . 57

Chapter 1: The Intersection Behaviour of the Curves $T_{N} \ldots \ldots 6$

1.1. Special Points . . . . . . . . . . . . . . . . . . . . . 60

1.2. Modules in Imaginary Quadratic Fields . . . . . . . . . . 68

1.3. The Transversal Intersections of the Curves $T_{N} \ldots$. . . . . 74

1.4. Contributions from the Cusps . . . . . . . . . . . 78

1.5. Self-Intersections . . . . . . . . . . . . . . . . . 82

Chapter 2: Modular Forms Whose Fourier Coefficients Involve Class Numbers . . . . . . . . . . . . . . . . . . . . . 87

2.1. The Modular Form $\varphi_{D}(z) \ldots \ldots$. . . . . . . . . . . . . . 88

2.2. The Eisenstein Series of Weight $\frac{3}{2}$. . . . . . . . . . . . . 91

2.3. A Theta-Series Attached to an Indefinite Quadratic Form . . 96

2.4. Proof of Theorem 1. . . . . . . . . . . . . . 100

Chapter 3: Modular Forms with Intersection Numbers as Fourier

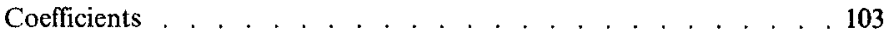

3.1. Modular Forms of Nebentypus and the Homology of the Hilbert Modular Surface . . . . . . . . . . . . . . . 103

3.2. The Relationship to the Doi-Naganuma Mapping . . . . . 108

References. . . . . . . . . . . . . . 111

\section{Introduction}

Let $p \equiv 1(\bmod 4)$ be a prime, $\mathcal{O}$ the ring of integers of $\mathbb{Q}(\sqrt{p})$. The group $S L_{2}(\mathcal{O})$ operates on the product of the upper half-plane with itself by

$$
\left(\begin{array}{ll}
\alpha & \beta \\
\gamma & \delta
\end{array}\right)\left(z_{1}, z_{2}\right)=\left(\frac{\alpha z_{1}+\beta}{\gamma z_{1}+\delta}, \frac{\alpha^{\prime} z_{2}+\beta^{\prime}}{\gamma^{\prime} z_{2}+\delta^{\prime}}\right) \quad\left(z_{1}, z_{2} \in \mathfrak{H}\right) .
$$

The quotient $X=\mathfrak{H}^{2} / S L_{2}(\mathcal{O})$ is a non-compact complex surface with finitely many singularities. On $X$ we define a series of curves $T_{1}, T_{2}, \ldots$ as follows:

* Sonderforschungsbereich „Theoretische Mathematik“ 
given $N$, we consider all points $\left(z_{1}, z_{2}\right) \in \mathfrak{S}^{2}$ satisfying some equation of the form

$$
a \sqrt{p} z_{1} z_{2}+\lambda z_{2}-\lambda^{\prime} z_{1}+b \sqrt{p}=0
$$

with $a, b \in \mathbb{Z}, \lambda \in \mathcal{O}, \lambda \lambda^{\prime}+a b p=N$; this set is invariant under $S L_{2}(\mathcal{O})$, and $T_{N}$ denotes its image in $X$. Then $T_{N}$ has finitely many components, is non-empty if $\left(\frac{N}{p}\right) \neq-1$, and is compact if $N$ is not the norm of an ideal of $\mathcal{O}$. The curves $T_{M}$ and $T_{N}$ meet transversally if $M N$ is not a square, and (because $X$ is a rational homology manifold) have a well-defined intersection number $T_{M} T_{N} \in \mathbb{Q}$. This number is evaluated in Chapter 1. We find for example $T_{1} T_{N}=H_{p}(N)$ if $N$ is not a square, where $H_{p}(N)$ is the arithmetical function

$$
H_{p}(N)=\sum_{\substack{x^{2} \leq 4 N \\ x^{2} \equiv 4 \bar{N}(\bmod p)}} H\left(\left(4 N-x^{2}\right) / p\right),
$$

$H(k)$ being the class number of positive definite binary quadratic forms of discriminant $-k$ (precise definitions are given in the paper). We also consider the compact surface $\tilde{X}$ obtained by adding to $X$ the "cusps" and resolving the singularities thus created (cf. [4]). The compactification of the curve $T_{N}$ represents a cycle in the middle homology group $H_{2}(\tilde{X})$. This group decomposes canonically as the direct sum of the image of $H_{2}(X)$ and the subspace generated by the homology cycles of the curves of the cusp resolution; we denote by $T_{N}^{c}$ the component of $T_{N}$ in the first summand. Then the intersection number of $T_{1}^{c}$ and $T_{N}^{c}$ on $\tilde{X}$ is the sum of $H_{p}(N)$ and a contribution $I_{p}(N)$ from the cusps given by

$$
I_{p}(N)=\frac{1}{\sqrt{p}} \sum_{\substack{\lambda \in \mathcal{O} \\ \lambda \geqslant 0 \\ \lambda \lambda^{\prime}=N}} \min \left(\lambda, \lambda^{\prime}\right)
$$

(this is a convergent series). The calculation of $T_{M}^{c} T_{N}^{c}$ on $\tilde{X}$ in general is more complicated, especially if $(M, N)>1$; we find for example

$$
\left(T_{M}^{c} T_{N}^{c}\right)_{\tilde{X}}=\sum_{d \mid(M, N)}\left(\frac{d}{p}\right) d\left(H_{p}\left(\frac{M N}{d^{2}}\right)+I_{p}\left(\frac{M N}{d^{2}}\right)\right)
$$

if $\left(\frac{M}{p}\right)=1$ (if $M N$ is a square, then $T_{M}$ and $T_{N}$ have components in common and we must calculate self-intersection numbers, but the formula remains valid).

In Chapter 2 we prove that the function

$$
\varphi_{p}(z)=-\frac{1}{12}+\sum_{N=1}^{\infty}\left(H_{p}(N)+I_{p}(N)\right) e^{2 \pi i N z} \quad(z \in \mathfrak{H})
$$

is a modular form of weight 2, level $p$, and "Nebentypus" (in Hecke's terminology), i.e.

$$
\varphi_{p}\left(\frac{a z+b}{c z+d}\right)=\left(\frac{a}{p}\right)(c z+d)^{2} \varphi_{p}(z)
$$


for all $z \in \mathfrak{H}$ and all $\left(\begin{array}{ll}a & b \\ c & d\end{array}\right) \in \Gamma_{0}(p)$. (Indeed, this is proved for $p$ the discriminant of any real quadratic field, not necessarily prime.) Thus the intersection numbers $\left(T_{1}^{c} T_{N}^{c}\right)_{\tilde{X}}$ are the Fourier coefficients of a modular form. The above formula for $\left(T_{M}^{c} T_{N}^{c}\right)_{\tilde{X}}$ shows that this number (if $p \nmid M$ ) is precisely the $N$-th Fourier coefficient of the modular form $\varphi_{p} \mid T(M)$, where $T(M)$ denotes a Hecke operator on the space $M_{2}\left(\Gamma_{0}(p),\left(\frac{-}{p}\right)\right)$ of modular forms of weight 2 , level $p$ and Nebentypus. In this way we obtain a map from the part of $H_{2}(\tilde{X} ; \mathbb{C})$ generated by the $T_{M}^{c}$ into this space of modular forms, and indeed into the subspace $M_{2}^{+}\left(\Gamma_{0}(p),\left(\frac{-}{p}\right)\right)$ (of half the dimension) of modular forms having the property that the $N$-th Fourier coefficient is zero whenever $\left(\frac{N}{p}\right)=-1$. We conjecture that this map is always an isomorphism (this has been verified for $p<200$ ). This conjecture, as well as the significance of the mapping and its relationship to the Doi-Naganuma lifting $([37,38])$, is discussed in Chapter 3.

The starting point for this paper was a letter of Serre (December 8, 1971), in which he pointed out that the number $\left[\frac{p+19}{24}\right]([$ ] $=$ greatest-integer function), which had occurred in a formula for the arithmetic genus of a surface related to $X$, is precisely the dimension of the space $M_{2}^{+}\left(\Gamma_{0}(p),\left(\frac{-}{p}\right)\right)$ as calculated by Hecke. To explain this coincidence, we calculated $\left(T_{M}^{c} T_{N}^{c}\right)_{\tilde{X}}$ in hundreds of cases on the Hewlett-Packard calculator at the I.H.E.S. - at the time we knew the formula for these intersection numbers only under the hypotheses $(M, N)=1$ and $T_{N}$ compact - and found empirically that these were Fourier coefficients of modular forms. Later we were able to prove this, as well as to extend the formulas for $T_{M} T_{N}$ to the general case $(M, N)>1$ and to determine the contribution of the cusps. Part of this work was done during visits to the University of California at Berkeley, the Collège de France, and the Institut des Hautes Etudes Scientifiques. The authors enjoyed the stimulating atmosphere at these institutions and would like to thank them for their hospitality.

The results concerning modular forms (Chapter 2) are due to the second author and were announced in [35]. Some of the results concerning the intersection numbers of the $T_{N}$ were announced in [5].

Notation. $K$ denotes a fixed real quadratic field, $\mathcal{O}$ its ring of integers, $x^{\prime}$, $\mathrm{N}(x)=x x^{\prime}$ and $\operatorname{Tr}(x)=x+x^{\prime}(x \in K)$ the conjugate, norm and trace of $x$, respectively; $x \gg 0$ means that $x$ is totally positive (i.e. $x>0$ and $x^{\prime}>0$ ). In Chapters 1 and 3 we assume that the discriminant of $K$ is a prime $p \equiv 1(\bmod 4)$; in Chapter 2, $K$ is arbitrary. We write $\chi_{d}(n)$ for the character associated to a quadratic field of discriminant $d, L\left(s, \chi_{d}\right)=\sum \chi_{d}(n) n^{-s}$ for the associated $L$-series. Thus $\chi_{p}(n)=\left(\frac{n}{p}\right)$. 
$\mathfrak{H}$ denotes the upper half-plane $\{z \in \mathbb{C} \mid \operatorname{Im}(z)>0\}, S L_{2}(\mathbb{Z})$ the group of $2 \times 2$ integral matrices of determinant 1 , acting on $\mathfrak{H}$ in the usual way, $\Gamma_{0}(N)$ the subgroup of matrices $\left(\begin{array}{ll}a & b \\ c & d\end{array}\right)$ with $N \mid c, S L_{2}(\mathcal{O})$ the group of $2 \times 2$ matrices with entries in $\mathcal{O}$ and determinant 1 , acting on $\mathfrak{H} \times \mathfrak{S}$ as in (1) above.

\section{Chapter 1: The Intersection Behaviour of the Curves $T_{N}$}

In this chapter we evaluate the intersection number of the curves $T_{M}$ and $T_{N}$ defined in the Introduction for all values of $M$ and $N$. We have

$$
T_{N}=\bigcup_{n^{2} \mid N} F_{N / n^{2}}
$$

where $F_{N} \subset X=\mathfrak{H}^{2} / S L_{2}(\mathcal{O})$ is defined as in (2) of the Introduction but with the additional requirement that only triples $(a, b, \lambda) \in \mathbb{Z} \times \mathbb{Z} \times \mathcal{O}$ are allowed which are not divisible by any natural number $>1$. All intersections of $F_{M}$ and $F_{N}(M \neq N)$ are transversal and occur at certain distinguished points of $X$ which we call "special". To each special point $z \in X$ is associated a positive definite binary quadratic form $\varphi_{3}$, and $T_{M}$ and $T_{N}$ meet in $z$ if and only if the form $\varphi_{3}$ represents both $M$ and $N$. In Section 1.1 we study the special points and determine how often any given positive definite form $\varphi$ occurs as the form $\varphi_{z}$ associated to some special $\mathfrak{z}$. This result, together with some results on representation by quadratic forms given in 1.2, permits the evaluation of the number of intersections of $T_{M}$ and $T_{N}$ on $X$ (Section 1.3). In the last sections we study how the curves $T_{N} \subset \tilde{X}$ meet the curves of the cusp resolution and evaluate the self-intersection numbers of the curves $T_{N}$ on $\tilde{X}$ for all $N$, thus obtaining in all cases a formula for the intersection number of the homology classes $\left[T_{M}\right]$ and $\left[T_{N}^{c}\right]$ on the compact suface $\tilde{X}$.

We remark that curves defined by skew-hermitian matrices have been previously used by Hammond [2].

\subsection{Special Points}

We fix a prime $p \equiv 1(\bmod 4)$ and consider the field $K=\mathbb{Q}(\sqrt{p})$ and the quaternary lattice $\mathfrak{M}$ of all skew-hermitian matrices

$$
A=\left(\begin{array}{cc}
a \sqrt{p} & \lambda \\
-\lambda^{\prime} & b \sqrt{p}
\end{array}\right) \quad(a, b \in \mathbb{Z}, \lambda \in \mathcal{O}),
$$

where $\mathcal{O}$ is the ring of algebraic integers contained in $K$. On $\mathfrak{M}$ we have the quadratic form

$$
\mathfrak{M} \ni A \mapsto \operatorname{det} A=a b p+\lambda \lambda^{\prime}
$$

of discriminant $p^{3}$. Over $\mathbb{R}$ this form is of type $(+,-,+,-)$. For a point $z=\left(z_{1}, z_{2}\right) \in \mathfrak{H} \times \mathfrak{S}$ let $\mathfrak{M}_{z}$ be the sublattice of $\mathfrak{M}$ consisting of all $A \in \mathfrak{M}$ such that

$$
a \sqrt{p} z_{1} z_{2}+\lambda z_{2}-\lambda^{\prime} z_{1}+b \sqrt{p}=0 \text {. }
$$


Equation (3) is equivalent for $A \neq 0$ to

$$
z_{2}=\frac{\lambda^{\prime} z_{1}-b \sqrt{p}}{a \sqrt{p} z_{1}+\lambda}
$$

Thus $\operatorname{det} A>0$ if $A \neq 0$, and $\mathfrak{M}_{z}$ is a sublattice of $\mathfrak{M}$ of rank 0,1 or 2 on which the function det is positive definite.

We can also introduce the 4-dimensional real vector space $\mathfrak{M} \otimes \mathbb{R}$ and associate to every point $z \in \mathfrak{S} \times \mathfrak{H}$ the 2-dimensional subspace $(\mathfrak{M} \otimes \mathbb{R})_{z}$ of $\mathfrak{M} \otimes \mathbb{R}$ consisting of all matrices $A$ satisfying (3) where $a, b, \lambda, \lambda^{\prime}$ are now four arbitrary real numbers. Then we get an embedding of $\mathfrak{S} \times \mathfrak{H}$ as an open subset of the Grassmannian $\mathfrak{G}$ of all 2-dimensional subspaces of $\mathfrak{M} \otimes \mathbb{R}$. It has the Grassmannian $\hat{\mathfrak{G}}$ of all oriented 2-dimensional subspaces of $\mathfrak{M} \otimes \mathbb{R}$ as a twofold cover. Since $\mathfrak{H} \times \mathfrak{H}$ is simply connected, we have two embeddings of $\mathfrak{H} \times \mathfrak{H}$ in $(\mathfrak{G}$ covering the embedding in $\boldsymbol{( \mathfrak { b }}$. One such embedding we choose once for all.

The lattice $\mathfrak{M}_{z}$ is contained in the oriented 2-dimensional real vector space $(\mathfrak{M} \otimes \mathbb{R})_{z}$. We call the point $z$ special if $\operatorname{rk} \mathfrak{M}_{z}=2$. Then $\varphi_{z}=\operatorname{det} \mid \mathfrak{M}_{z}$ is a positive definite binary integral quadratic form defined over an oriented lattice. Clearly, $\mathfrak{M} / \mathfrak{M}_{z}$ is torsion-free and $\mathfrak{M}_{z}$ is a direct summand of $\mathfrak{M}$. Therefore, for every prime $q$, the 2-dimensional vector space $\mathfrak{M}_{z} \otimes \mathbb{Z} / q \mathbb{Z}$ over $\mathbb{Z} / q \mathbb{Z}$ is a direct summand of $\mathfrak{M} \otimes \mathbb{Z} / q \mathbb{Z}$. In the next lemma, we will state exactly which binary quadratic forms can occur as forms $\varphi_{z}$. First we introduce some terminology concerning binary quadratic forms.

For an oriented $\mathbb{Z}$-lattice $L$ of rank 2 we use only bases $e_{1}, e_{2}$ of $L$ compatible with the orientation. A quadratic form $\varphi: L \rightarrow \mathbb{Z}$ can be written as

$$
\varphi\left(u e_{1}+v e_{2}\right)=\alpha u^{2}+\beta u v+\gamma v^{2},
$$

where $\alpha=\varphi\left(e_{1}\right), \gamma=\varphi\left(e_{2}\right), \beta=\varphi\left(e_{1}+e_{2}\right)-\varphi\left(e_{1}\right)-\varphi\left(e_{2}\right)$. Such a quadratic form will sometimes be denoted by $[\alpha, \beta, \gamma]$. The discriminant of $\varphi$ is $\beta^{2}-4 \alpha \gamma$. The content of $\varphi$ is defined as the greatest common divisor $(\alpha, \beta, \gamma)$. It is the greatest common divisor of all integers represented by $\varphi$. A form of content 1 is called primitive. Isomorphisms (equivalences) between quadratic forms are always supposed to be orientation preserving. Thus $\left[\alpha_{1}, \beta_{1}, \gamma_{1}\right]$ and $\left[\alpha_{2}, \beta_{2}, \gamma_{2}\right]$ are equivalent if and only if one can be transformed into the other by an element of $S L_{2}(\mathbb{Z})$. We sometimes speak of $S L_{2}(\mathbb{Z})$-equivalence.

Lemma 1. Let $z$ be special. Then the discriminant of $\varphi_{z}: \mathfrak{M}_{z} \rightarrow \mathbb{Z}$ is divisible by $p$ and $\varphi_{z}$ represents only quadratic residues modulo $p$. The content $m$ of $\varphi_{z}$ is not divisible by $p^{2}$ or by any prime $q$ with $\left(\frac{q}{p}\right)=-1$. If $m$ is divisible by $p$ and the discriminant of $\frac{1}{p} \varphi_{z}$ is also divisible by $p$, then $\frac{1}{p} \varphi_{z}$ represents only quadratic residues
$\bmod p$.

Proof. Since $\mathfrak{M}_{z} \otimes \mathbb{Z} / p \mathbb{Z}$ is a direct summand of $\mathfrak{M} \otimes \mathbb{Z} / p \mathbb{Z}$ and the quadratic form det has a 3-dimensional nullspace on the latter space, the discriminant of $\varphi_{z}=\operatorname{det} \mid \mathfrak{M}_{z}$ must be divisible by $p$; that $\varphi_{z}$ represents only quadratic residues $\bmod p$ is clear from the definition and Equation (2). If the odd prime $q$ divides $m$, then, choosing a basis for the vector space $\mathfrak{M} \otimes \mathbb{Z} / q \mathbb{Z}$ whose first two elements 
span $\mathfrak{M}_{z} \otimes \mathbb{Z} / q \mathbb{Z}$, we see that the form 2 det on $\mathfrak{M} \otimes \mathbb{Z} / q \mathbb{Z}$ can be given by a symmetric integral matrix $\left(\begin{array}{l|l}0 & C \\ \hline C^{t} & B\end{array}\right)$, where $B$ and $C$ are $2 \times 2$ matrices. Thus, because the form det on $\mathfrak{M}$ has discriminant $p^{3}$,

$$
p^{3} \equiv(\operatorname{det} C)^{2}(\bmod q) \text {, }
$$

so $\left(\frac{q}{p}\right) \neq-1$.

We now prove that $m \neq 0(\bmod 2)$ if $p \equiv 5(\bmod 8)$ and that $m \equiv 0\left(\bmod p^{2}\right)$ is not possible. Let $A_{1}, A_{2}$ be a base of $\mathfrak{M}_{z}$, with $A_{i}$ a matrix (1) given by $\left(a_{i}, b_{i}, \lambda_{i}\right)$. Then $\varphi_{z}$ has content divisible by $r$ if and only if

$$
\operatorname{det} A_{1} \equiv \operatorname{det} A_{2} \equiv \operatorname{det}\left(A_{1}+A_{2}\right) \equiv 0(\bmod r)
$$

or

(i) $a_{1} b_{1} p+\lambda_{1} \lambda_{1}^{\prime} \equiv 0(\bmod r)$,

(ii) $a_{2} b_{2} p+\lambda_{2} \lambda_{2}^{\prime} \equiv 0(\bmod r)$,

(iii) $\left(a_{1} b_{2}+a_{2} b_{1}\right) p+\left(\lambda_{1} \lambda_{2}^{\prime}+\lambda_{2} \lambda_{1}^{\prime}\right) \equiv 0(\bmod r)$.

Suppose $r=2$ and $p \equiv 5(\bmod 8)$. Write $\lambda_{i}=c_{i}+d_{i} \frac{1+\sqrt{p}}{2}$. Then (i), (ii) and (iii) become $($ since $(1-p) / 4 \equiv 1(\bmod 2))$

(i) $a_{1} b_{1} \equiv c_{1}+d_{1}+c_{1} d_{1}(\bmod 2)$,

(ii)' $a_{2} b_{2} \equiv c_{2}+d_{2}+c_{2} d_{2}(\bmod 2)$,

(iii)' $a_{1} b_{2}+a_{2} b_{1} \equiv c_{1} d_{2}+c_{2} d_{1}(\bmod 2)$.

Equation (i)' has five non-zero solutions

$$
\left(a_{1}, b_{1}, c_{1}, d_{1}\right) \equiv(1,0,0,0),(0,1,0,0),(1,1,1,0),(1,1,0,1),(1,1,1,1)
$$

modulo 2 and similarly for (ii)'. Since $\left(a_{i}, b_{i}, c_{i}, d_{i}\right)(i=1,2)$ must span a 2dimensional vector space over $\mathbb{Z} / 2 \mathbb{Z}$, the two vectors must be different from each other and from 0 . This is not compatible with (iii)'.

Now suppose $r=p^{2}$. Write $\lambda_{i}=\frac{1}{2}\left(c_{i}+d_{i} \sqrt{p}\right)$. Then $c_{i} \equiv 0(\bmod p)$ and so (i)-(iii) become
(i)" $a_{1} b_{1} \equiv \frac{1}{4} d_{1}^{2}(\bmod p)$,
(ii)" $a_{2} b_{2} \equiv \frac{1}{4} d_{2}^{2}(\bmod p)$,
(iii)" $a_{1} b_{2}+a_{2} b_{1} \equiv \frac{1}{2} d_{1} d_{2}(\bmod p)$.

Since $\left(a_{1}, b_{1}, d_{1}\right)$ and $\left(a_{2}, b_{2}, d_{2}\right)$ are not proportional $\bmod p$, we have a twodimensional subspace of $(\mathbb{Z} / p \mathbb{Z})^{3}$ on which the non-degenerate form $a b-d^{2} / 4$ vanishes identically. This is impossible.

Finally, suppose that $p \mid m$ and that $\frac{1}{p} \varphi_{z}$ has a discriminant divisible by $p$. If, as before, $A_{1}, A_{2}$ is a base of $\mathfrak{M}_{z}$ and $\lambda_{i}=\frac{1}{2}\left(c_{i}+d_{i} \sqrt{p}\right)$, then $c_{i} \equiv 0(\bmod p)$ and $\left(a_{1}, b_{1}, d_{1}\right)$ and $\left(a_{2}, b_{2}, d_{2}\right)$ span a direct summand $\mathfrak{N}$ of $(\mathbb{Z} / p \mathbb{Z})^{3}$. On the 3dimensional vector space $(\mathbb{Z} / p \mathbb{Z})^{3}$ we have the non degenerate form $\psi(a, b, d)=$ $a b-d^{2} / 4$ of discriminant $1 / 2\left(=\right.$ determinant of the matrix $\left.\left(\begin{array}{rrr}0 & 1 & 0 \\ 1 & 0 & 0 \\ 0 & 0 & -\frac{1}{2}\end{array}\right)\right)$ 
with $\psi \mid \mathfrak{N}=\frac{1}{p} \varphi_{z}$. We use a splitting $(\mathbb{Z} / p \mathbb{Z})^{3} \cong \mathfrak{N} \oplus \mathbb{Z} / p \mathbb{Z}$ and diagonalize $2 \psi \mid \mathfrak{N}$. Then the form $2 \psi(\bmod p)$ is represented by a matrix

$$
\left(\begin{array}{cc|c}
0 & 0 & u \\
0 & 2 \alpha & v \\
\hline u & v & w
\end{array}\right)
$$

(the upper left $2 \times 2$ matrix represents $2 \psi \mid \mathfrak{N}$, which has vanishing determinant $\bmod p$ and therefore a zero on the diagonal). Thus

$$
\frac{1}{2} \equiv-2 \alpha u^{2}(\bmod p)
$$

and $\alpha$ is a quadratic residue $\bmod p$, so the form $\frac{1}{p} \varphi_{z} \equiv \psi \mid \mathfrak{P} \equiv[0,0, \alpha](\bmod p)$ represents only quadratic residues. This completes the proof of the lemma.

The Hilbert modular group $S L_{2}(\mathcal{O})$ operates on $\mathfrak{S} \times \mathfrak{5}$. Every $B \in S L_{2}(\mathcal{O})$ induces an automorphism of $\mathfrak{M}$ :

$$
A \mapsto\left(B^{\prime}\right)^{t} A B
$$

which carries $\mathfrak{M}_{B z}$ to $\mathfrak{M}_{z}$ preserving the orientation of these lattices and giving an equivalence of the quadratic forms $\varphi_{B z}$ and $\varphi_{z}$. Thus we can speak of special points on the Hilbert modular surface $\mathfrak{H}^{2} / S L_{2}(\mathcal{O})$. They are represented by special points of $\mathfrak{H}^{2}$. For $z \in \mathfrak{S}^{2} / S L_{2}(\mathcal{O})$ represented by $z \in \mathfrak{S}^{2}$ write $\varphi_{3}$ instead of $\varphi_{2}$, where, however, the form $\varphi_{3}$ is defined only up to equivalence. We wish to count the number of special points of $\mathfrak{H}^{2} / S L_{2}(\mathcal{O})$ for which $\varphi_{3}$ is (equivalent to) a given form $\varphi$. As number of special points 3 with $\varphi_{3} \cong \varphi$ we define

$$
s(\varphi)=w_{\varphi} \sum_{\substack{\mathfrak{z} \in \mathfrak{H}^{2} / S L_{2}(\varphi) \\ \varphi_{3} \cong \varphi}} \frac{1}{v_{3}},
$$

where $v_{3}$ is the order of the isotropy group of $S L_{2}(\mathcal{O})$ at a point $z \in \mathfrak{G}^{2}$ representing 3 and $w_{\varphi}$ the order of the group Aut $(\varphi)$ of orientation-preserving automorphisms of $\varphi$. Of course, $v_{3} / 2$ is the order of the isotropy group of the effectively acting group $S L_{2}(\mathcal{O}) /\{ \pm 1\}$. The possible values of $v_{3} / 2$ are 1,2 and 3 (and 5 for $p=5$ ). The possible values of $w_{\varphi} / 2$ are 1,2 and 3 .

The main result of this section is the following theorem. We first recall that $h(\Delta)$ denotes the number of equivalence classes of primitive positive definite binary integral quadratic forms with discriminant $\Delta$ (for $\Delta<0$ with $\Delta \equiv 0$ or 1 $(\bmod 4))$. By $h^{\prime}(4)$ we denote the modified class number where the class of a form $\varphi$ is counted with multiplicity $2 / w_{\varphi}$. Thus $h^{\prime}(-3)=\frac{1}{3}, h^{\prime}(-4)=\frac{1}{2}$ and $h^{\prime}(\Delta)=h(\Delta)$ otherwise.

Theorem 1. Let $\varphi$ be a positive definite quadratic form of discriminant $\Delta \equiv 0(\bmod p)$ and content $m$. Let $\varphi_{0}=\frac{1}{m} \varphi$ be the corresponding primitive form, $\Delta_{0}=\Delta / m^{2}$ its discriminant. Then

$$
s(\varphi)=\frac{1}{2}\left(1+\chi_{p}\left(\varphi_{0}\right)\right) \beta_{p}(m) h^{\prime}(\Delta / p),
$$


where

and

$$
\chi_{p}\left(\varphi_{0}\right)=\left\{\begin{aligned}
0 & \text { if } p \nmid \Delta_{0}, \\
1 & \text { if } p \mid \Delta_{0} \text { and } \varphi_{0} \text { represents only quadratic residues }(\bmod p), \\
-1 & \text { otherwise }
\end{aligned}\right.
$$

$$
\beta_{p}(m)= \begin{cases}\prod_{q \mid m}\left(1+\chi_{p}(q)\right) & \text { if } p^{2} \nmid m \\ 0 & \text { if } p^{2} \mid m\end{cases}
$$

The cases where $s(\varphi)=0$ by (7) are already proved in Lemma 1 . The proof of Theorem 1 will be preceded by a lemma concerning the $\Gamma_{0}(N)$-classification of quadratic forms.

Lemma 2. Let $N>0, \Delta<0, m>0$ be given with $\Delta \equiv 0$ or $1(\bmod 4), m\left|N, m^{2}\right| \Delta$, $N / m$ square-free and prime to $m$. Then the number of $\Gamma_{0}(N)$-equivalence classes of positive definite quadratic forms $[a N, b, c]$ with

$$
(a, b, c)=1, \quad b^{2}-4 a c N=\Delta, \quad(N, b, a c)=m,
$$

each form $\varphi$ being counted with multiplicity $2 /\left|\operatorname{Aut}(\varphi) \cap \Gamma_{0}(N)\right|$, is given by

$$
h^{\prime}(\Delta) 2^{v} \prod_{q}\left(1+\left(\frac{\Delta}{q}\right)\right) \text {, }
$$

where $v$ is the number of distinct prime factors of $m$ and the product is taken over all primes $q$ dividing $N / m$.

Proof. By (8), any prime dividing $m$ divides $a$ or $c$ but not both. Hence the numbers $(N, b, a)$ and $(N, b, c)$, which are easily seen to be $\Gamma_{0}(N)$-invariants of the form $[a N, b, c]$, are relatively prime and have product $m$. A further $\Gamma_{0}(N)$ invariant is the value of $b(\bmod 2 N)$. By $(8)$, this value is of the form $m x$ with $x$ a residue class $(\bmod 2 N / m)$ satisfying $x^{2} \equiv \Delta / m^{2}(\bmod 4 N / m)$. There are precisely $\prod_{q}\left(1+\left(\frac{\Delta}{q}\right)\right)$ such residue classes $x(\bmod 2 N / m)$ and $2^{\nu}$ decompositions $m=m_{1} m_{2}$ with $\left(m_{1}, m_{2}\right)=1$. Hence the lemma will be proved if we show that, for each decomposition $m=m_{1} m_{2}$ and each value of $x$ there are $h^{\prime}(\Delta) \Gamma_{0}(N)$-equivalence classes of forms $[a N, b, c]$ satisfying $(8)$ and

$$
(N, b, a)=m_{1}, \quad(N, b, c)=m_{2}, \quad b \equiv m x(\bmod 2 N) .
$$

To do this, we define a map from the set of such forms to the set of primitive forms of discriminant $\Delta$ by

$$
\varphi=[a N, b, c] \mapsto \tilde{\varphi}=\left[a N_{1}, b, c N_{2}\right],
$$

where we have written $N=N_{1} N_{2}$ with $\left(N_{1}, N_{2}\right)=1$ and $N_{2}$ containing exactly those prime divisors of $N$ which divide $m_{2}$. Acting on $\varphi$ with $\left(\begin{array}{cc}\alpha & \beta \\ \gamma N & \delta\end{array}\right)$ corresponds to acting on $\tilde{\varphi}$ with $\left(\begin{array}{cc}\alpha & \beta N_{2} \\ \gamma N_{1} & \delta\end{array}\right)$; in particular, the $\Gamma_{0}(N)$-equivalence class of $\varphi$ determines the $S L_{2}(\mathbb{Z})$-equivalence class of $\tilde{\varphi}$. Conversely, if 
$\varphi_{1}, \varphi_{2}$ are two forms satisfying (8) and (9), and $\left(\begin{array}{ll}A & B \\ C & D\end{array}\right) \in S L_{2}(\mathbb{Z})$ transforms $\tilde{\varphi}_{1}$ to $\tilde{\varphi}_{2}$, then an easy calculation shows that $N_{1} \mid C$ and $N_{2} \mid B$, so $\varphi_{1}$ is transformed to $\varphi_{2}$ by the matrix $\left(\begin{array}{cc}A & B / N_{2} \\ C N_{2} & D\end{array}\right) \in \Gamma_{0}(N)$. Finally, any primitive form of discriminant $\Delta$ is equivalent under $S L_{2}(\mathbb{Z})$ to $\tilde{\varphi}$ for some $\varphi$ satisfying (8) and (9) (this is just a matter of checking congruences modulo the various prime powers dividing $N$ ). Therefore $\varphi \mapsto \tilde{\varphi}$ is a $1: 1$ correspondence between $\Gamma_{0}(N)$-equivalence classes of forms $[a N, b, c]$ satisfying $(8)$ and $(9)$ and $S L_{2}(\mathbb{Z})$-equivalence classes of primitive forms of discriminant $\Delta$, with $\left|\operatorname{Aut}(\varphi) \cap \Gamma_{0}(N)\right|=|\operatorname{Aut}(\tilde{\varphi})|$. This proves the lemma.

Proof of Theorem 1. By Lemma 1, we may suppose that $m$ is not divisible by $p^{2}$ or by any prime $q$ with $\left(\frac{q}{p}\right)=-1$ and that $\chi_{p}\left(\varphi_{0}\right)=0$ or 1 . Then $\varphi_{0}$ is a primitive form which represents quadratic residues $(\bmod p)$, so by a classical theorem $[11,14]$ it represents a prime $q_{0}$ with $q_{0} \nmid \Delta,\left(\frac{q_{0}}{p}\right)=1$. Then $\varphi$ represents the number $N=m q_{0}$ primitively, so all special points $\mathfrak{z}$ with $\varphi_{3} \cong \varphi$ lie on the curve $F_{N}$ defined at the beginning of the chapter. The curve $F_{N}$ in $\mathfrak{S}^{2} / S L_{2}(\mathcal{O})$ is irreducible [7] and can be described as follows:

The number $N$ is the norm of a primitive ideal $\mathbf{b}$ in $\mathcal{O}$ (i.e. one which is not divisible by any natural number $>1$ ). Consider the group $S L_{2}(\mathcal{O}, \mathbf{b})$ of matrices of determinant 1 belonging to $\left(\begin{array}{cc}\mathcal{O} & \mathfrak{b}^{-1} \\ \mathfrak{b} & \mathcal{O}\end{array}\right)$. This is the group of automorphisms of $\mathscr{O} \oplus \mathfrak{b}$. Since the class number of $K=\mathbb{Q}(\sqrt{p})$ is odd, every ideal class is a square, so $\mathcal{O} \oplus \mathfrak{b}$ is $G L_{2}^{+}(K)$-equivalent to $\mathcal{O} \oplus \mathcal{O}$. This sets up a canonical isomorphism

$$
\rho: \mathfrak{H}^{2} / S L_{2}(\mathcal{O}, \mathfrak{b}) \rightarrow \mathfrak{H}^{2} / S L_{2}(\mathcal{O})
$$

([4] 5.3), and $F_{N}$ is the image under $\rho$ of the curve in $\mathfrak{S}^{2} / S L_{2}(\mathcal{O}$, b) given by the diagonal in $\mathfrak{S}^{2}$. For $t \in \mathfrak{H}$, we sometimes write simply $t$ for the image $\rho(t, t)$ ) of $(t, t)$ in $F_{N}$. The subgroup $\Gamma$ of $S L_{2}(\mathcal{O}, \mathfrak{b})$ carrying the diagonal to itself is $\Gamma_{0}(N)$ if $p \nmid N$ and an extension of $\Gamma_{0}(N)$ of index 2 if $p \mid N$. We have a map $\mathfrak{H} / \Gamma \rightarrow F_{N}$ of degree one.

On the other hand, $\mathfrak{M}$ is the lattice of skew-hermitian forms taking integral values on $\mathcal{O} \oplus \mathcal{O}$, so it corresponds under the above equivalence to the lattice of all skew-hermitian forms taking integral values on $\mathcal{O} \oplus$ b, i.e. to the lattice of all matrices

$$
A=\left(\begin{array}{cc}
a \sqrt{p} & \lambda \\
-\lambda^{\prime} & \frac{c}{N} \sqrt{p}
\end{array}\right) \quad\left(a, c \in \mathbb{Z}, \lambda \in \mathfrak{b}^{-1}\right),
$$

where for technical reasons we have replaced $b$ in (1) by $c$. The quadratic form (2) on $\mathfrak{M}$ is transformed to

$$
\mathrm{A} \mapsto N(\mathfrak{b}) \operatorname{det} A=a c p+N \lambda \lambda^{\prime} .
$$


A point $t \in \mathfrak{H}$ corresponds to a special point of $F_{N}$ if and only if it satisfies a quadratic equation

$$
a_{0} N t^{2}+b_{0} t+c_{0}=0, \quad\left(a_{0}, b_{0}, c_{0}\right)=1 .
$$

Then a matrix $A$ as in (10) belongs to the lattice $\mathfrak{M}_{t}$ if and only if

$$
a N t^{2}+N \frac{\lambda-\lambda^{\prime}}{\sqrt{p}} t+c=0
$$

and this is equivalent to

$$
a=x a_{0}, \quad N \frac{\lambda-\lambda^{\prime}}{\sqrt{p}}=x b_{0}, \quad c=x c_{0} \quad(x \in \mathbb{Z}) .
$$

Also, $\lambda^{\prime} \in \mathfrak{b}^{\prime-1}=N^{-1} \mathfrak{b}$, and the ideal $\mathfrak{b}$ can be written as

$$
\mathbf{b}=\mathbb{Z} \frac{M-\sqrt{p}}{2}+\mathbb{Z} N
$$

for some integer $M$ with $M^{2} \equiv p(\bmod 4 N)$. Therefore

$$
N \lambda=x b_{0} \frac{M+\sqrt{p}}{2}+y N
$$

with $y \in \mathbb{Z}$ and

$$
a c p+N \lambda \lambda^{\prime}=x^{2}\left(a_{0} c_{0} p+b_{0}^{2} \frac{M^{2}-p}{4 N}\right)+x y b_{0} M+y^{2} N .
$$

This means that the quadratic form $\varphi_{t}$ of a special point $t$ as in (12) is given by

$$
\left[a_{0} c_{0} p+b_{0}^{2} \frac{M^{2}-p}{4 N}, b_{0} M, N\right] \text {. }
$$

The discriminant of this form is $p \Delta_{1}$, where $\Delta_{1}=b_{0}^{2}-4 a_{0} c_{0} N$ is the discriminant of the quadratic polynomial (12). We have to look for those points $t \in \mathfrak{H}$ satisfying an Equation (12) for which the form (13) is equivalent to our given form $\varphi$.

To simplify the exposition, we suppose first that the form $\varphi$ has no nontrivial automorphisms and is not the form $\varphi_{3}$ for any of the finitely many quotient singularities $z \in X$, i.e. that $w_{\varphi}=v_{3}=2$ in Equation (6). We consider first the case $p \nmid m$. Then $p \nmid N$ and $(N, M)=1$, so the content of the form (13) is $\left(N, b_{0}, a_{0} c_{0}\right)$. Also, $\Gamma=\Gamma_{0}(N)$ in this case. By Lemma 2, there are $h^{\prime}(\Delta) 2^{v}\left(1+\left(\frac{\Delta_{1}}{q_{0}}\right)\right) \Gamma_{0}(N)$ equivalence classes of forms $\left[a_{0} N, b_{0}, c_{0}\right]$ with $\left(a_{0}, b_{0}, c_{0}\right)=1,\left(N, b_{0}, a_{0} c_{0}\right)=m$, $b_{0}^{2}-4 a_{0} c_{0} N=\Delta_{1}=\Delta / p$, where $v$ is the number of distinct prime factors of $m$. We have $\left(\frac{\Delta_{1}}{q_{0}}\right)=\left(\frac{\Delta}{q_{0}}\right)=1$, so there are two $S L_{2}(\mathbb{Z})$-inequivalent representations of $q_{0}$ by primitive forms of discriminant $\Delta / \mathrm{m}^{2}$. Thus one of two cases occurs:

(a) The form $\varphi$ chosen originally is the only form (up to $S L_{2}(\mathbb{Z})$-equivalence) of discriminant $\Delta$ and content $m$ which represents $N=m q_{0}$, and $\varphi$ represents $N$ twice; or 
(b) There are two forms of discriminant $\Delta$ and content $m$ which represent $N$, namely the form $\varphi$ and a form improperly equivalent to $\varphi$ (i.e. $G L_{2}(\mathbb{Z})$ - but not $S L_{2}(\mathbb{Z})$-equivalent), and each represents $N$ exactly once.

In case (a), the $2 h^{\prime}\left(\Delta_{1}\right) 2^{v}$ points $t \in \mathfrak{H} / \Gamma$ given by (12) are mapped in pairs to $h^{\prime}\left(\Delta_{1}\right) 2^{v}$ points of $F_{N}$, because the fact that $\varphi$ represents $N$ twice means that each point $z \in X$ with $\varphi_{3} \cong \varphi$ is a double point of $F_{N}$. In case (b) the $2 h^{\prime}\left(\Delta_{1}\right) 2^{v}$ points $t$ are mapped to distinct points of $F_{N}$, but for only half of these points is the corresponding form $\varphi_{3}$ equivalent to $\varphi$. Thus in both cases we find $2^{v} h^{\prime}\left(\Delta_{1}\right)=$ $\beta_{p}(m) h^{\prime}(\Delta / p)$ points $3 \in F_{N}$ with $\varphi_{3} \cong \varphi$, in accordance with (7).

If $p \mid m$, then $(N, M)=p$ and $p \nmid \frac{M^{2}-p}{4 N}$. Also $p^{2} \mid \Delta$, so $p \mid \Delta_{1}$. The content of the form (13) is either $\left(N, b_{0}, a_{0} c_{0}\right)$ or $p\left(N, b_{0}, a_{0} c_{0}\right)$, according as $p$ does or does not divide the number $\Delta_{0}=\Delta / m^{2}$. By Lemma 2 , there are $h^{\prime}\left(\Delta_{1}\right) 2^{v}\left(1+\left(\frac{\Delta_{1}}{q_{0}}\right)\right)$ $\Gamma_{0}(N)$-equivalence classes of forms $\left[a_{0} N, b_{0}, c_{0}\right]$ with this value of $\left(N, b_{0}, a_{0} c_{0}\right)$, where $v$ is now the number of prime factors of $\left(N, b_{0}, a_{0} c_{0}\right)$. Clearly $2^{v}=$ $\left(1+\chi_{p}\left(\varphi_{0}\right)\right) \beta_{p}(m)$. The rest of the argument is as in the case $p \nmid m$, except that we must divide the final result by $\left|\Gamma: \Gamma_{0}(N)\right|=2$. This proves formula (7) in this case, too.

It remains to discuss the isotropy and automorphism groups. In the definition of $s(\varphi)$ (Eq. (6)), we count each point 3 with $\varphi_{3} \cong \varphi$ with multiplicity $w_{\varphi} / v_{3}$, where $w_{\varphi}=|\operatorname{Aut}(\varphi)|$ and $v_{3}=\left|S L_{2}(\mathcal{O})_{3}\right|$. There is an obvious map $\pi: S L_{2}(\mathcal{O})_{3} \rightarrow \operatorname{Aut}(\varphi)$ (compare (5)), and

$$
\frac{w_{\varphi}}{v_{z}}=\frac{|\operatorname{Aut}(\varphi)|}{|\operatorname{Im}(\pi)|} \cdot \frac{1}{|\operatorname{Ker}(\pi)|}
$$

The first factor represents the number of distinct $t \in \mathfrak{S} / \Gamma$ mapping onto 3 , i.e. the number of branches of $F_{N}$ passing through 3 (or rather, of those branches corresponding to a given $S L_{2}(\mathbb{Z})$-equivalence class of representations of $N$ by $\varphi$ ). The second factor $1 /|\operatorname{Ker}(\pi)|$ is just $2 /\left|\Gamma_{t}\right|$, where $\Gamma_{t}$ is the isotropy group of $t$ in $\Gamma$. But in Lemma 2, which we used for our proof of Theorem 1, each $\Gamma_{0}(N)$ equivalence class of forms was counted in precisely this way, and the same applies to the passage from $\Gamma_{0}(N)$ to $\Gamma$ in case $p \mid m$. Thus the proof given above counts each point $z$ in just the way required by (6).

Example. Prestel [8] found that there are $h(-3 p)$ quotient singularities of order 3 on $X, h(-4 p)$ of order 2 and two of order 5 if $p=5$. Consider a form $\varphi$ with discriminant $\Delta=-3 p$ (respectively $-4 p$ ) and content 1 which represents quadratic residues $(\bmod p)$. There are $\frac{1}{2} h(-3 p)\left(\right.$ resp. $\left.\frac{1}{2} h(-4 p)\right)$ such forms. By Theorem 1 , $s(\varphi)=\frac{1}{3}$ (resp. $\frac{1}{2}$ ), and since $w_{\varphi}=2$ (because $\Delta<-4$ ) this can only mean that $v_{3}=6$ (resp. 4). Thus we have found half of the fixed points of order 3 (resp. 2). Now consider the form $\varphi=[p, p, p]$ of discriminant $-3 p^{2}$ and content $p$ (resp. $\varphi=[p, 0, p]$ of discriminant $-4 p^{2}$ and content $p$ ). Here Theorem 1 gives $s(\varphi)=$ $\frac{1}{2} \beta_{p}(p) h^{\prime}(-3 p)=\frac{1}{2} h(-3 p)$ (resp. $\frac{1}{2} h(-4 p)$ ). Here $w_{\varphi}=6$ (resp. 4) and one can check that $v_{3}=w_{\varphi}$, so $s(\varphi)$ is the actual number of special points 3 with $\varphi_{3} \cong \varphi$, 
and these points are all quotient singularities of order 3 (resp. 2). We have thus found the remaining fixed points of order 2 or 3.

The two fixed points of order 5 when $p=5$ are not special.

\subsection{Modules in Imaginary Quadratic Fields ${ }^{1}$}

Let $k$ be an imaginary quadratic field and $M$ a module in $k$, i.e. a free $\mathbb{Z}$-module of rank 2 contained in $k$. Two modules $M_{1}, M_{2}$ are called equivalent if there exists an element $\alpha$ of $k$ such that $\alpha M_{1}=M_{2}$. For a module $M$ the norm $\mathrm{N}(M)$ can be characterized as the unique rational number such that the quadratic form

$$
x \mapsto \frac{x \bar{x}}{\mathrm{~N}(M)} \quad(x \in M, \bar{x}=\text { conjugate of } x)
$$

is integral and primitive. An order of $k$ is a module which is a subring of the ring $\mathfrak{o}$ of all algebraic integers in $k$ and contains 1 . For every natural number $f$ there is a unique order $\mathfrak{o}_{f}$ with $\left|\mathfrak{0}: \mathbf{o}_{f}\right|=f$. The number $f$ is called the conductor of the order. For a module $M$ the set of all $\alpha \in k$ with $\alpha M \subset M$ is an order $\mathfrak{D}(M)$. The conductor $f$ of this order is also called the conductor of $M$. Thus $\mathfrak{v}(M)=\mathfrak{o}_{f}$. The quadratic form given in (14) has discriminant $D f^{2}$ where $D$ is the discriminant of $k$. Every module is oriented by the bases $z, w$ with $z / w \in \mathfrak{H}$. We have a bijection (using (14)) between the set of equivalence classes of modules of discriminant $\Delta$ and the set of isomorphism classes of primitive positive definite integral quadratic forms of discriminant $\Delta$. We have $\Delta=D f^{2}$ where $D$ is the discriminant of a field $k$ (discriminants of fields are called fundamental discriminants). The number of equivalence classes of modules of discriminant $\Delta$ is the class number $h(\Delta)$. Every module is equivalent to a module admitting a base $z, 1$ with $z \in \mathfrak{H}$. In this way we get a bijection between the set of equivalence classes of all modules in all imaginary quadratic fields and the set of $S L_{2}(\mathbb{Z})$-equivalence classes of points in $\mathfrak{S}$ which satisfy a quadratic equation over $\mathbb{Q}$. If $\varphi$ is a primitive form, $M$ a corresponding module having the $\mathbb{Z}$-base $z, 1(z \in \mathfrak{S})$, then the group Aut $(\varphi)$, the group $\operatorname{Aut}(M)$ of units of $\mathfrak{o}(M)$ and the isotropy group of $z$ in $S L_{2}(\mathbb{Z})$ are isomorphic. Of course, for a form of content $m$ we have $\operatorname{Aut}(\varphi)=\operatorname{Aut}\left(\frac{1}{m} \varphi\right)$.

Of fundamental importance for this paper is the function $H(n)$ defined for $n>0$ as the number of equivalence classes of all positive definite forms of discriminant $-n$, where the equivalence classes of $m[1,0,1]$ and $m[1,1,1]$ are counted with multiplicity $\frac{1}{2}$ and $\frac{1}{3}$ respectively. Of course, $H(n)=0$ if $-n$ is not a discriminant. For reasons which will become apparent later, we define $H(0)$ $=-\frac{1}{12}$. The function $H(n)$ occurs in the work of Kronecker and Hurwitz. We can also define $H(n)$ as the number of $S L_{2}(\mathbb{Z})$-equivalence classes of points $z \in \mathfrak{H}$ which satisfy a quadratic equation over $\mathbb{Z}$ with discriminant $-n$,

$$
\alpha z^{2}+\beta z+\gamma=0, \quad \beta^{2}-4 \alpha \gamma=-n,
$$

1 A general reference for modules in quadratic fields is [9]. Results related to Propositions 2 and 3 below are given in [13] and [10] 
where $(\alpha, \beta, \gamma)$ is arbitrary and where a point equivalent to $i$ or to $e^{\pi i / 3}$ is counted $\frac{1}{2}$ or $\frac{1}{3}$, respectively. We have

$$
H(n)=\sum_{d^{2} \mid n} h^{\prime}\left(-n / d^{2}\right),
$$

where $d$ runs through those natural numbers such that $-n / d^{2}$ is a discriminant.

Two modules $M_{1}, M_{2} \subset k$ can be multiplied. If $f_{1}$ is the conductor of $M_{1}$ and $f_{2}$ of $M_{2}$, then $f=\left(f_{1}, f_{2}\right)$ is the conductor of $M_{1} M_{2}$. We have $\mathrm{N}\left(M_{1} M_{2}\right)=$ $\mathrm{N}\left(M_{1}\right) \mathrm{N}\left(M_{2}\right)$. The equivalence classes of modules of fixed conductor $f$ constitute a group $G\left(D f^{2}\right)$ of order $h\left(D f^{2}\right)$, where $D$ is again the discriminant of $k$. For every divisor $d$ of $f$ the map

$$
M \mapsto \mathfrak{o}_{d} M
$$

induces a homomorphism of $G\left(D f^{2}\right)$ onto $G\left(D d^{2}\right)$ which can be used to prove the following well-known proposition.

Proposition 1. Let $D$ be a fundamental discriminant. Then

$$
h^{\prime}\left(D f^{2}\right)=h^{\prime}(D) \gamma_{D}(f),
$$

where

$$
\gamma_{D}(f)=f \prod_{q \mid f}\left(1-\left(\frac{D}{q}\right) / q\right)
$$

and $q$ runs through all primes dividing $f$.

Let $r_{A}(n)$ denote the number of $S L_{2}(\mathbb{Z})$-inequivalent representations of the natural number $n$ by all primitive forms of discriminant $\Delta$. For $\Delta=D$ a fundamental discriminant, the following formula is well-known $([9,12])$ :

$$
r_{D}(n)=\sum_{d \mid n}\left(\frac{D}{d}\right)
$$

However, we need the formula for general discriminant $\Delta=D f^{2}$; for convenience of notation we write $r_{D}(f, n)$ instead of $r_{D f^{2}}(n)$ and $\chi_{D}(n)$ instead of $\left(\frac{D}{n}\right)$.

Proposition 2. Let $D$ be a fundamental discriminant. Then $r_{D}(f, n)$ is given as follows:

(a) $r_{D}(f, n)$ is simultaneously multiplicative, i.e. if $f=\prod q_{i}^{\alpha_{i}}$ and $n=\prod q_{i}^{\beta_{i}}$ (where the $q_{i}$ are distinct primes), then

$r_{D}(f, n)=\prod r_{D}\left(q_{i}^{\alpha_{1}}, q_{i}^{\beta_{2}}\right)$.

(b) For q prime, introduce the generating series

$$
R_{D, q}(t, u)=\sum_{\alpha=0}^{\infty} \sum_{\beta=0}^{\infty} r_{D}\left(q^{\alpha}, q^{\beta}\right) t^{\alpha} u^{\beta}
$$

in indeterminates $t, u$. Then we have

$$
R_{D, q}(t, u)=\frac{(1-t u)\left(1-\chi_{D}(q) t u\right)}{(1-t)(1-u)\left(1-q t u^{2}\right)\left(1-\chi_{D}(q) u\right)} .
$$


Equivalently,

$$
r_{D}\left(q^{\alpha}, q^{\beta}\right)=\left\{\begin{array}{cl}
q^{\beta / 2} & \text { if } \beta<2 \alpha, \beta \text { even, } \\
0 & \text { if } \beta<2 \alpha, \beta \text { odd }, \\
\gamma_{D}\left(q^{\alpha}\right) \sum_{r=0}^{\beta-2 \alpha} \chi_{D}(q)^{r} & \text { if } \beta \geqq 2 \alpha .
\end{array}\right.
$$

Proof. Let $r_{D}^{*}(f, n)$ denote the number of $S L_{2}(\mathbb{Z})$-inequivalent primitive representations of $n$ by all forms of discriminant $D f^{2}$, i.e. we now count representations $n=a x^{2}+b x y+c y^{2}$ (where $b^{2}-4 a c=D f^{2}$ ) with $(x, y)=1$, whereas for $r_{D}(f, n)$ we count those with $(a, b, c)=1$. Clearly

$$
\sum_{d \mid(f, n)} r_{D}(f / d, n / d)=\sum_{c^{2} \mid n} r_{D}^{*}\left(f, n / c^{2}\right),
$$

since both sides count the total number of representations of $n$ by forms of discriminant $D f^{2}$, with no condition of primitivity on the form or on the representation. But it is well-known [12] that

$$
r_{D}^{*}(f, n)=\#\left\{k(\bmod 2 n) \mid k^{2} \equiv D f^{2}(\bmod 4 n)\right\},
$$

and from this formula it follows easily that $r_{D}^{*}(f, n)$ is simultaneously multiplicative in $f, n$ in the sense of (a), which together with (20) implies the multiplicativity of $r_{D}$. It remains to determine $r_{D}\left(q^{\alpha}, q^{\beta}\right)$ for $q$ prime.

We introduce the generating series

$$
R_{D, q}^{*}(t, u)=\sum_{\alpha=0}^{\infty} \sum_{\beta=0}^{\infty} r_{D}^{*}\left(q^{\alpha}, q^{\beta}\right) t^{\alpha} u^{\beta}
$$

Then formula (20) is equivalent to the identity

$$
(1-t u)^{-1} R_{D, q}(t, u)=\left(1-u^{2}\right)^{-1} R_{D, q}^{*}(t, u) .
$$

From (21) we see that $r_{D}^{*}\left(q^{\alpha}, q^{\beta}\right)=q r_{D}^{*}\left(q^{\alpha-1}, q^{\beta-2}\right)$ if $\alpha \geqq 1$ and $\beta \geqq 2$. By (19),

$$
r_{D}\left(1, q^{\beta}\right)=\sum_{r=0}^{\beta} \chi_{D}(q)^{r}
$$

whence

$$
R_{D, q}(0, u)=(1-u)^{-1}\left(1-\chi_{D}(q) u\right)^{-1}
$$

and (by (22))

$$
R_{D, q}^{*}(0, u)=(1+u) /\left(1-\chi_{D}(q) u\right),
$$

whereas (21) implies $r_{D}^{*}\left(q^{\alpha}, 1\right)=r_{D}^{*}\left(q^{\alpha}, q\right)=1$. Hence

$$
\begin{aligned}
R_{D, q}^{*}(t, u) & =\frac{1+u}{1-\chi_{D}(q) u}+\frac{t(1+u)}{1-t}+\sum_{\alpha=1}^{\infty} \sum_{\beta=2}^{\infty} r_{D}^{*}\left(q^{\alpha}, q^{\beta}\right) t^{\alpha} u^{\beta} \\
& =\frac{(1+u)\left(1-\chi_{D}(q) u t\right)}{\left(1-\chi_{D}(q) u\right)(1-t)}+q t u^{2} R_{D, q}^{*}(t, u)
\end{aligned}
$$


Thus

$$
R_{D, q}^{*}(t, u)=\frac{(1+u)\left(1-\chi_{D}(q) u t\right)}{\left(1-\chi_{D}(q) u\right)(1-t)\left(1-q t u^{2}\right)},
$$

and the formula for $R_{D, q}$ in (b) follows by (22).

Remark. The contents of Proposition 2 can be summarized by the formula

$$
\sum_{f=1}^{\infty} \sum_{n=1}^{\infty} \frac{r_{D}(f, n)}{f^{a} n^{s}}=\prod_{q \text { prime }} R_{D, q}\left(q^{-a}, q^{-s}\right)=\frac{\zeta(s) \zeta(a) \zeta(2 s+a-1) L\left(s, \chi_{D}\right)}{\zeta(s+a) L\left(s+a, \chi_{D}\right)} .
$$

We also have to study the representation of forms by forms. We therefore introduce for modules $M_{1}, M_{2}$ in $k$ of conductors $f_{1}, f_{2}$ the module

$\operatorname{Hom}\left(M_{1}, M_{2}\right)=\left\{\alpha \in k \mid \alpha M_{1} \subset M_{2}\right\}$

on which we have the quadratic form

$$
\varphi_{M_{1}, M_{2}}(\alpha)=\left|M_{2}: \alpha M_{1}\right| \text {. }
$$

Lemma 3. The content of the quadratic form $\varphi_{M_{1}, M_{2}}$ equals

$$
m=f_{1} f_{2} / f^{2}
$$

where $f=\left(f_{1}, f_{2}\right)$. The primitive quadratic form $\frac{1}{m} \varphi_{M_{1}, M_{2}}$ belongs to the module $\bar{M}_{1} M_{2}$ (see (14)).

Proof. We can check easily that

and

$$
\operatorname{Hom}\left(M_{1}, M_{2}\right)=\frac{f_{2}}{f} \frac{1}{\mathrm{~N}\left(M_{1}\right)} \bar{M}_{1} M_{2}
$$

$$
\varphi_{M_{1}, M_{2}}(\alpha)=\frac{\mathrm{N}\left(M_{1}\right) f_{1}}{\mathrm{~N}\left(M_{2}\right) f_{2}} \alpha \bar{\alpha} .
$$

Let $\psi$ be a quadratic form of discriminant $D f_{1}^{2} m_{1}^{2}$ and content $m_{1}$ (where $D$ is a fundamental discriminant). Define $r_{D}\left(\psi ; m_{2}, f_{2}\right)$ as the number of $S L_{2}(\mathbb{Z})$ inequivalent representations of $\psi$ by forms of discriminant $D f_{2}^{2} m_{2}^{2}$ and content $m_{2}$. If $\psi$ is the quadratic form

$$
x \mapsto \psi(x)=m_{1} \frac{x \bar{x}}{\mathrm{~N}\left(M_{1}\right)} \quad\left(x \in M_{1}\right),
$$

then such a representation of $\psi$ is given by a module $M_{2} \subset k=\mathbb{Q}(\sqrt{D})$ of conductor $f_{2}$ and an element $\alpha \in \operatorname{Hom}\left(M_{1}, M_{2}\right)$ with

$$
\left|M_{2}: \alpha M_{1}\right|=\varphi_{M_{1}, M_{2}}(\alpha)=\frac{m_{1} f_{1}}{m_{2} f_{2}} .
$$

Thus $r_{D}\left(\psi ; m_{2}, f_{2}\right)$ is the number of pairs $\left(M_{2}, \alpha\right)$ with $\alpha \in \operatorname{Hom}\left(M_{1}, M_{2}\right)$ satisfying (24), where $\left(M_{2}, \alpha\right)$ and $\left(\tilde{M}_{2}, \tilde{\alpha}\right)$ are considered equal if there exists an element $\gamma \in k$ such that $\gamma M_{2}=\tilde{M}_{2}$ and $\tilde{\alpha}=\gamma \alpha$. 
Proposition 3. The number $r_{D}\left(\psi ; m_{2}, f_{2}\right)$ depends only on $m_{1}, f_{1}$, where $D f_{1}^{2} m_{1}^{2}$ is the discriminant and $m_{1}$ the content of $\psi$. We write $r_{D}\left(m_{1}, f_{1} ; m_{2}, f_{2}\right)$ instead of $r_{D}\left(\psi ; m_{2} f_{2}\right)$. Then

$$
r_{D}\left(m_{1}, f_{1} ; m_{2}, f_{2}\right)=r_{D}\left(f, \frac{m_{1}}{m_{2}} \frac{f^{2}}{f_{2}^{2}}\right) \frac{\gamma_{D}\left(f_{2}\right)}{\gamma_{D}(f)} .
$$

where $f=\left(f_{1}, f_{2}\right)$ and $r_{D}(f, n)=0$ if $n$ is not an integer.

Proof. By $M_{2} \mapsto \bar{M}_{1} M_{2}$ with fixed $M_{1}$ we get a map $G\left(D f_{2}^{2}\right) \rightarrow G\left(D f^{2}\right)$. Since $\bar{M}_{1} M_{2}=\mathrm{o}_{f} \bar{M}_{1} \cdot \mathrm{o}_{f} M_{2}$, we can conclude from (16) that the inverse image of any element of $G\left(D f^{2}\right)$ has $\left|G\left(D f_{2}^{2}\right)\right| /\left|G\left(D f^{2}\right)\right|$ elements. By (17) and (18),

$$
\left|G\left(D f_{2}^{2}\right)\right| /\left|G\left(D f^{2}\right)\right|=\frac{\gamma_{D}\left(f_{2}\right)}{\gamma_{D}(f)} \frac{\left|\operatorname{Aut}\left(M_{2}\right)\right|}{\left|\operatorname{Aut}\left(\bar{M}_{1} M_{2}\right)\right|} .
$$

We have to count the "number" of pairs $\left(M_{2}, \alpha\right)$ with $\alpha \in \operatorname{Hom}\left(M_{1}, M_{2}\right)$ such that (24) holds. By Lemma 3 this is just the number of pairs $\left(M_{2}, \alpha\right)$ with $\alpha \in \bar{M}_{1} M_{2}$ such that

$$
\frac{\alpha \bar{\alpha}}{\mathrm{N}\left(M_{1}\right) \mathrm{N}\left(M_{2}\right)}=\frac{m_{1}}{m_{2}} \frac{f^{2}}{f_{2}^{2}} .
$$

The "number" of possible $\alpha$ equals $r_{D}\left(f, \frac{m_{1}}{m_{2}} \frac{f^{2}}{f_{2}^{2}}\right) \cdot \operatorname{Aut}\left(\bar{M}_{1} M_{2}\right)$. To get the "number" of pairs $\left(M_{2}, \alpha\right)$ we have to multiply this with the expression in (25) and divide by $\left|\operatorname{Aut}\left(M_{2}\right)\right|$.

For the application of Propositions 2 and 3 to Hilbert modular surfaces we will need some additional information concerning the behaviour of quadratic forms at our fixed prime $p$. If $M$ is a module in an imaginary quadratic field $k$ and $\varphi_{0}$ the corresponding primitive quadratic form, we set $\chi_{p}(M)=\chi_{p}\left(\varphi_{0}\right)$, where $\chi_{p}\left(\varphi_{0}\right)$ was defined in Theorem 1. Thus $\chi_{p}(M)=0$ if the discriminant $\Delta$ of $M$ is not divisible by $p$ and $\chi_{p}(M)= \pm 1$ if $p \mid \Delta$. The character $\chi_{p}$ is multiplicative on the modules of $k$.

Now consider a discriminant $\Delta=D f^{2}$ which is divisible by $p$ and write $\Delta / p=D_{0} g^{2}$ (here $D, D_{0}$ are fundamental discriminants, $D_{0}=D / p$ or $D p$ ). We define $r_{D}^{+}(f, n), r_{D}^{-}(f, n)$ as the number of $S L_{2}(\mathbb{Z})$-inequivalent representations of $n$ by primitive forms of discriminant $\Delta$ and character $\chi_{p}$ equal to +1 or -1 , respectively. We write

$$
r_{D}^{+}(f, n)-r_{D}^{-}(f, n)=r_{D}^{(p)}(g, n) .
$$

The function $r_{D}^{(p)}$ is simultaneously multiplicative in $g, n$ (not in $f, n$ ). For each prime $q$ we define the generating series $R_{D, q}^{(p)}(t, u)=\sum r_{D}^{(p)}\left(q^{\alpha}, q^{\beta}\right) t^{\alpha} u^{\beta}$. Obviously

$$
R_{D, q}^{(p)}(t, u)=R_{D, q}\left(t, \chi_{p}(q) u\right) \quad \text { if } q \neq p .
$$

For $p=q$ one has

$$
R_{D, p}^{(p)}(t, u)=\frac{1-\chi_{D_{0}}(p) t u}{(1-t)\left(1-\chi_{D_{0}}(p) u\right)\left(1-p t u^{2}\right)} .
$$

The proof of these assertions is analogous to that of Proposition 2. 
Let $\psi$ be a quadratic form of discriminant $D f_{1}^{2} m_{1}^{2}$ and content $m_{1}$ with $p \mid D f_{1}^{2}$. We define $r_{D}^{(p)}\left(\psi ; m_{2}, f_{2}\right)$ as the number of $S L_{2}(\mathbb{Z})$-inequivalent representations of $\psi$ by quadratic forms $\varphi$ of discriminant $D m_{2}^{2} f_{2}^{2}$ and content $m_{2}$, where each $\varphi$ is counted with multiplicity $\chi_{p}\left(\psi / m_{1}\right) \chi_{p}\left(\varphi / m_{2}\right)$ (note that this is zero unless $\left.p \mid D f_{2}^{2}\right)$.

Proposition 3'. The number $r_{D}^{(p)}\left(\psi ; m_{2}, f_{2}\right)$ depends only on $m_{1}, f_{1}$ where $D f_{1}^{2} m_{1}^{2}$ is the discriminant and $m_{1}$ the content of $\psi$. It is 0 (by definition) if $D f_{1}^{2}$ and $D f_{2}^{2}$ are not both divisible by $p$. If $p \mid D f_{1}^{2}$ and $p \mid D f_{2}^{2}$, write $D f_{1}^{2}=D_{0} g_{1}^{2} p$ and $D f_{2}^{2}=$ $D_{0} g_{2}^{2} p$ and also $r_{D}^{(p)}\left(m_{1}, g_{1} ; m_{2}, g_{2}\right)$ instead of $r_{D}^{(p)}\left(\psi ; m_{2}, f_{2}\right)$. Then

$$
r_{D}^{(p)}\left(m_{1}, g_{1} ; m_{2}, g_{2}\right)=r_{D}^{(p)}\left(g, \frac{m_{1}}{m_{2}} \frac{g^{2}}{g_{2}^{2}}\right) \frac{\gamma_{D}\left(f_{2}\right)}{\gamma_{D}(f)},
$$

where $g=\left(g_{1}, g_{2}\right)$.

The proof is the same as for Proposition 3. We use the multiplicativity of $\chi_{p}$ on modules.

Let $\psi$ be a quadratic form of discriminant $D f_{1}^{2} m_{1}^{2}$ and content $m_{1}$. Define $\rho_{D}\left(\psi ; m_{2}, f_{2}\right)$ as the number of $S L_{2}(\mathbb{Z})$-inequivalent representations of $\psi$ by forms of discriminant $D f_{2}^{2} m_{2}^{2}$ and content $m_{2}$, where now each $\varphi$ is counted with multiplicity $\frac{1}{2}\left(1+\chi_{p}\left(\varphi / m_{2}\right)\right)$. Then

$$
\rho_{D}\left(\psi ; m_{2}, f_{2}\right)=\left\{\begin{aligned}
\frac{1}{2} r_{D}\left(m_{1}, f_{1} ; m_{2}, f_{2}\right) & \text { unless } p\left|D f_{1}^{2}, p\right| D f_{2}^{2}, \\
\frac{1}{2}\left(r_{D}\left(m_{1}, f_{1} ; m_{2}, f_{2}\right)+\chi_{p}\left(\psi / m_{1}\right) r_{D}^{(p)}\left(m_{1}, g_{1} ; m_{2}, g_{2}\right)\right) & \text { if } D f_{1}^{2}=D_{0} g_{1}^{2} p, D f_{2}^{2}=D_{0} g_{2}^{2} p .
\end{aligned}\right.
$$

These equations follow easily from the definitions and from the proof of Proposition 3. In the case $p \nmid D f_{1}^{2}$, but $p \mid D f_{2}^{2}$, we have to use that in the kernel of $G\left(D f_{2}^{2}\right) \rightarrow G\left(D f^{2}\right)$ the elements with $\chi_{p}=1$ form a subgroup of index 2 .

For the calculations concerning Hilbert modular surfaces it is convenient to give the simultaneously multiplicative functions $r_{D}\left(m_{1}, f_{1} ; m_{2}, f_{2}\right)$ and $r_{D}^{(p)}\left(m_{1}, g_{1} ; m_{2}, g_{2}\right)$ by generating series. These functions depend only on the ratio $m_{1} / m_{2}$ (and of course $f_{1}, f_{2}$ or $g_{1}, g_{2}$ ). For each prime $q$ we write

$$
T_{q}(x, y, z)=\sum_{r, s, l} r_{D}\left(q^{r}, q^{s} ; 1, q^{l}\right) x^{r} y^{s} z^{l}
$$

Since by Proposition 3

$$
r_{D}\left(q^{r}, q^{s} ; 1, q^{l}\right)= \begin{cases}r_{D}\left(q^{l}, q^{r}\right) & \text { for } s \geqq l, \\ r_{D}\left(q^{s}, q^{r+2 s-2 l}\right) q^{l-s} & \text { for } 0<s<l, \\ r_{D}\left(1, q^{r-2 l}\right) q^{l}\left(1-\chi_{D}(q) / q\right) & \text { for } 0=s<l,\end{cases}
$$

we have

$$
T_{q}(x, y, z)=T_{q}^{\prime}(x, y, z)+T_{q}^{\prime \prime}(x, y, z)-T_{q}^{\prime \prime \prime}(x, y, z),
$$

where

$$
T_{q}^{\prime}(x, y, z)=\sum_{\substack{r, s, l \\ s \geqq l}} r_{D}\left(q^{l}, q^{r}\right) x^{r} y^{s} z^{l}=\frac{1}{1-y} R_{D, q}(y z, x)
$$


(compare Proposition 2(b)),

$$
\begin{aligned}
& T_{q}^{\prime \prime}(x, y, z)=\sum_{\substack{r, s, l \\
0 \leqq s<l}} r_{D}\left(q^{s}, q^{r+2 s-2 l}\right) q^{l-s} x^{r} y^{s} z^{l}, \\
& T_{q}^{\prime \prime \prime}(x, y, z)=\chi_{D}(q) \sum_{\substack{r, l \\
l>0}} r_{D}\left(1, q^{r-2 l}\right) q^{l-1} x^{r} z^{l} .
\end{aligned}
$$

To evaluate $T_{q}^{\prime \prime}$ we put $l-s=j$ and $r-2 j=k$ and obtain

$$
T_{q}^{\prime \prime}(x, y, z)=\sum_{\substack{j, k, s \\ j>0}} r_{D}\left(q^{s}, q^{k}\right) q^{j} x^{k+2 j} y^{s} z^{j+s}=R_{D, q}(y z, x) \frac{q x^{2} z}{1-q x^{2} z} .
$$

Similarly,

$$
T_{q}^{\prime \prime \prime}(x, y, z)=\chi_{D}(q) R_{D, q}(0, x) \frac{x^{2} z}{1-q x^{2} z} .
$$

Thus we have

$$
T_{q}(x, y, z)=R_{D, q}(y z, x) \frac{1-q x^{2} y z}{(1-y)\left(1-q x^{2} z\right)}-\chi_{D}(q) R_{D, q}(0, x) \frac{x^{2} z}{1-q x^{2} z}
$$

where $R_{D, q}$ is given in Proposition 2. In the same way we calculate the generating series $T_{p}^{(p)}(x, y, z)=\sum r_{D}^{(p)}\left(p^{r}, p^{s} ; 1, p^{l}\right) x^{r} y^{s} z^{l}$ and obtain from Proposition $3^{\prime}$ and (26)

$$
\begin{aligned}
T_{p}^{(p)}(x, y, z) & =R_{D, p}^{(p)}(y z, x) \frac{1-p x^{2} y z}{(1-y)\left(1-p x^{2} z\right)} \\
& =\frac{1-\chi_{D_{0}}(p) x y z}{(1-z y)\left(1-\chi_{D_{0}}(p) x\right)(1-y)\left(1-p x^{2} z\right)} .
\end{aligned}
$$

\subsection{The Transversal Intersections of the Curves $T_{N}$}

Let $\psi$ be a positive definite form defined over the oriented lattice $\mathbb{Z} \oplus \mathbb{Z}$ and let $s_{0}(\psi)$ be the number of orientation-preserving embeddings $j: \mathbb{Z} \oplus \mathbb{Z} \rightarrow \mathfrak{M}_{z}$ with $z \in \mathfrak{G}^{2}$ special and $\varphi_{z} \circ j=\psi$, where $S L_{2}(\mathcal{O})$-equivalent embeddings are identified (cf. (5)). Each such embedding is counted with multiplicity $\frac{1}{|\operatorname{Ker}(\pi)|}$, where $\pi: S L_{2}(\mathcal{O})_{z} \rightarrow \operatorname{Aut}\left(\varphi_{z}\right)$ as in the proof of Theorem 1 is the map given by (5).

Theorem 2. Let $\psi$ be a positive definite quadratic form of discriminant $\Delta \equiv 0(\bmod p)$ and content $m$ and $\psi_{0}=\frac{1}{m} \psi$ the corresponding primitive form. Then

$$
s_{0}(\psi)=\sum_{d \mid m} \frac{1}{2}\left(\chi_{p}(d)+\chi_{p}\left(\frac{m}{d}\right) \chi_{p}\left(\psi_{0}\right)\right) d H\left(-\Delta / p d^{2}\right),
$$

where $H$ is defined in (15). (We put $H(n)=0$ if $n$ is not integral.)

Proof. We write $m_{1}$ instead of $m$ and $\Delta=D f_{1}^{2} m_{1}^{2}$, where $D$ is a fundamental discriminant, and again write $D_{0}$ for the discriminant of $Q(\sqrt{D p})$. Theorem 1 
implies

$$
s_{0}(\psi)=\sum_{m_{2}, f_{2}} \rho_{D}\left(m_{1}, f_{1} ; m_{2}, f_{2}\right) \beta_{p}\left(m_{2}\right) h^{\prime}\left(D m_{2}^{2} f_{2}^{2} / p\right),
$$

where $\rho_{D}$ was given in (27). We put $h^{\prime}(n)=0$ if $n$ is not integral. The reader may check that isotropy and automorphism groups are counted in the correct way. It suffices to prove

$$
\sum_{d \mid m_{1}} \chi_{p}(d) d H\left(-D f_{1}^{2} m_{1}^{2} / p d^{2}\right)=\sum_{m_{2}, f_{2}} r_{D}\left(m_{1}, f_{1} ; m_{2}, f_{2}\right) \beta_{p}\left(m_{2}\right) h^{\prime}\left(D m_{2}^{2} f_{2}^{2} / p\right)
$$

and, for $D f_{1}^{2}=D_{0} g_{1}^{2} p$,

$$
\begin{aligned}
& \sum_{d \mid m_{1}} \chi_{p}\left(m_{1} / d\right) d H\left(-D_{0} g_{1}^{2} m_{1}^{2} / d^{2}\right) \\
& \quad=\sum_{m_{2}, g_{2}} r_{D}^{(p)}\left(m_{1}, g_{1} ; m_{2}, g_{2}\right) \beta_{p}\left(m_{2}\right) h^{\prime}\left(D_{0} g_{2}^{2} m_{2}^{2}\right)
\end{aligned}
$$

Substituting for $H$ from (15) we can rewrite the left-hand sides of (31) and (31') as sums where all summands have a factor $h^{\prime}\left(D_{0} a^{2}\right)$ which we substitute by $h^{\prime}\left(D_{0}\right) \gamma_{D_{0}}(a)$ (Proposition 1). The same can be done for $h^{\prime}\left(D m_{2}^{2} f_{2}^{2} / p\right)$ and $h^{\prime}\left(D_{0} g_{2}^{2} m_{2}^{2}\right)$ on the right. We divide both sides of (31) and (31') by $h^{\prime}\left(D_{0}\right)$. Then both sides of (31) become simultaneously multiplicative in $m_{1}, u_{1}$, where $D f_{1}^{2} m_{1}^{2}=D_{0} u_{1}^{2} p$, and both sides of $\left(31^{\prime}\right)$ simultaneously multiplicative in $m_{1}, g_{1}$. Thus we can treat both equations at each prime $q$ separately. We first prove (31) for the case $q \neq p$ or $q=p, p \nmid D_{0}$ (i.e. $p \mid D$ ). Then $p \mid D f_{1}^{2}$ and we can write $m_{1}=q^{r}$, $g_{1}=q^{s}$ in (31), where $D f_{1}^{2}=D_{0} g_{1}^{2} p$. We then write $a(r, s)$ for the left-hand side of (31) divided by $h^{\prime}\left(D_{0}\right)$. We put $\varepsilon=\chi_{p}(q)$ and $\alpha=\chi_{D_{0}}(q)$. We have

$$
a(r, s)=\sum_{k=0}^{r} \varepsilon^{k} q^{k} \sum_{n=0}^{r+s-k} \gamma_{D_{0}}\left(q^{n}\right)
$$

and therefore the generating series $A(x, y)=\sum a(r, s) x^{r} y^{s}$ is given by

$$
\begin{aligned}
A(x, y) & =\sum_{k, h, s \geqq 0} \varepsilon^{k} q^{k}\left(\sum_{n=0}^{h+s} \gamma_{D_{0}}\left(q^{n}\right)\right) x^{k+h} y^{s} \\
& =\frac{1}{1-\varepsilon q x} \sum_{h, s \geqq 0}\left(1+\left(1-\frac{\alpha}{q}\right)\left(q+q^{2}+\cdots+q^{h+s}\right)\right) x^{h} y^{s} \\
& =\frac{1}{1-\varepsilon q x} \sum_{h, s \geqq 0}\left(\frac{1-q^{h+s+1}}{1-q}-\alpha \frac{1-q^{h+s}}{1-q}\right) x^{h} y^{s} \\
& =\frac{1-\alpha x-\alpha y-q x y+\alpha x y+q \alpha x y}{(1-\varepsilon q x)(1-x)(1-y)(1-q x)(1-q y)} .
\end{aligned}
$$

On the right-hand side of (31) the summation runs over all $m_{2}=q^{k}, g_{2}=q^{l}$ with $D f_{2}^{2}=D_{0} g_{2}^{2} p$. For the coefficient $\beta_{p}\left(m_{2}\right) h^{\prime}\left(D_{0} g_{2}^{2} m_{2}^{2}\right)$ appearing in (31) we introduce the generating series $B(x, y)=\sum \beta_{p}\left(q^{k}\right) \gamma_{D_{0}}\left(q^{k+l}\right) x^{k} y^{l}$ and obtain

$$
B(x, y)=\frac{\alpha}{q}+\left(1-\frac{\alpha}{q}\right) \frac{1+q x}{1-\varepsilon q x} \frac{1}{1-q y} .
$$


The numbers $r_{D}\left(q^{r}, q^{s} ; q^{k}, q^{l}\right)$ occurring in (31) can be regarded as the coefficients of an infinite matrix $M$ giving an endomorphism of the $\mathbb{Q}$-vector space $\mathbb{Q}[[x, y]]$ which is $\mathbb{Q}[[x]]$-linear. We must prove $A=M B$. In view of (28) (noting also that $r_{D}\left(q^{r}, p q^{s} ; 1, p q^{l}\right)=r_{D}\left(q^{r}, q^{s} ; 1, q^{l}\right)$ for $p \neq q$ and $\left.p \nmid D\right)$ we have to prove the identity

$$
A(x, y)=\frac{\alpha}{q} T_{q}(x, y, 0)+\left(1-\frac{\alpha}{q}\right) \frac{1+q x}{1-\varepsilon q x} T_{q}(x, y, q) .
$$

In the definition of $T_{q}$ enters $\delta=\chi_{D}(q)$, whereas the numbers $\varepsilon=\chi_{p}(q)$ and $\alpha=\chi_{D_{0}}(q)$ enter in (32) and (33). The reader may check that (34) is a formal identity in $x, y, q, \varepsilon, \alpha, \delta$ provided $\delta=\varepsilon \alpha$. This finishes the proof of (31) except in the case $p \nmid D$ (i.e. $D_{0}=D p$ ) and $p=q$, where we have to prove (31) for $m_{1}=p^{r}$, $f_{1}=p^{s}$ with $r+s>0$. The summation runs over all $m_{2}=p^{k}, f_{2}=p^{l}$ with $k+l>0$. If we define $a_{0}(r, s)$ as the left-hand side of (31) (for $m_{1}=p^{r}, f_{1}=p^{5}$ ) divided by $h^{\prime}\left(D_{0}\right)$ and $A_{0}(x, y)$ as the corresponding generating series we have

$$
A_{0}(x, y)=\frac{y+x-x y-p x y}{(1-x)(1-y)(1-p x)(1-p y)} .
$$

The generating series $B_{0}(x, y)=\sum \beta_{p}\left(p^{k}\right) \gamma_{D_{0}}\left(p^{k+l-1}\right) x^{k} y^{l}$ is given by

$$
p B_{0}(x, y)=\frac{1+p x}{1-p y}-1,
$$

and we must show that $A_{0}=M B_{0}$, i.e. we must check the identity

$$
p A_{0}(x, y)=(1+p x) T_{p}(x, y, p)-T_{p}(x, y, 0)
$$

(compare (34)), which is indeed true.

We now prove (31'). If $p \nmid m_{1}$, then $p \mid D f_{1}^{2}$ and $\left(31^{\prime}\right)$ is equivalent to (31). We consider (31') for $m_{1}=p^{r}, g_{1}=p^{s}$ and define $a^{\prime}(r, s)$ as the left-hand side divided by $h^{\prime}\left(D_{0}\right)$. Then the corresponding generating series $A^{\prime}(x, y)$ is given by

$$
A^{\prime}(x, y)=\frac{1-\alpha y}{(1-y)(1-p x)(1-p y)} .
$$

We have $B(x, y)$ as in (33) and must check the equality

$$
A^{\prime}(x, y)=\frac{\alpha}{p} T_{p}^{(p)}(x, y, 0)+\left(1-\frac{\alpha}{p}\right)(1+p x) T_{p}^{(p)}(x, y, p)
$$

in place of the previous (34). Using (28') this is a formal identity. Thus Theorem 2 is proved in all cases.

We are now finally able to evaluate the number of transversal intersections of the curves $T_{M}$ and $T_{N}$ on the Hilbert modular surface $X$. The curves $T_{M}, T_{N}$ have a common component if and only if $M N$ is a square. We do not exclude this case. A transversal intersection of $T_{M}$ and $T_{N}$ in a point $3 \in X$ represented by $z \in \mathfrak{H}^{2}$ is given by an ordered pair $(A, B)$ of linearly independent elements of $\mathfrak{M}_{z}$ (cf. 1.1) with $\operatorname{det} A=M, \operatorname{det} B=N$. Thus we have transversal intersections only in special points. Each ordered pair $(A, B)$ determines an orientation of $\mathfrak{M}_{z}$. 
On the other hand, in 1.1 an orientation of $\mathfrak{M}_{z}$ was chosen once for all. Let $\left(T_{M} T_{N}\right)_{z}$ be the transversal intersection number of $T_{M}$ and $T_{N}$ in $z$ in the sense of rational homology manifolds. Then $\left(T_{M} T_{N}\right)_{3}$ is $\frac{1}{v_{3}}$ times the number of ordered pairs $(A, B)$ with $A, B$ linearly independent elements of $\mathfrak{M}_{z}$ determining the given orientation and with $\varphi_{z}(A)=M, \varphi_{z}(B)=N$. (Note that the orientation convention allows only the sign change from $A, B$ to $-A,-B$ and that each $v_{z}$ contains the factor 2, with $v_{3} / 2$ the order of the isotropy group of $S L_{2}(\mathcal{O}) /\{ \pm 1\}$ at $z$. Because $S L_{2}(\mathcal{O}) /\{ \pm 1\}$ acts effectively on $\mathfrak{S}^{2}$, the intersection theory on rational homology manifolds requires division by $v_{3} / 2$.) For each such pair we get a quadratic form by restricting $\varphi_{z}$ to $\mathbb{Z} A \oplus \mathbb{Z} B$. This quadratic form has the form $[M, b, N]$. Also, it is positive definite and has discriminant divisible by $p$ (Lemma 1 ), so the integer $b$ must satisfy $4 M N-b^{2}>0,4 M N-b^{2} \equiv 0(\bmod p)$. Conversely, for any integer $b$ satisfying these two conditions we can consider the quadratic form $\psi=[M, b, N]$ over $\mathbb{Z} \oplus \mathbb{Z}$ and the number of $S L_{2}(\mathcal{O})_{z}$-inequivalent orientation-preserving embeddings $j: \mathbb{Z} \oplus \mathbb{Z} \rightarrow \mathfrak{M}_{z}$ with $\varphi_{z} \circ j=\psi$ (where each such embedding is counted with multiplicity $1 /|\operatorname{Ker}(\pi)|$ as explained at the beginning of this section). Clearly $\left(T_{M} T_{N}\right)_{3}$ is the sum of these numbers for all $b$. The transversal intersection number

$$
\left(T_{M} T_{N}\right)_{X}^{\mathrm{tr}}=\sum_{\substack{3 \in X \\ 3 \text { special }}}\left(T_{M} T_{N}\right)_{3}
$$

of $T_{M}$ and $T_{N}$ on $X$ is therefore given by the formula

$$
\left(T_{M} T_{N}\right)_{X}^{\mathrm{tr}}=\sum_{\substack{b \in \mathbb{Z} \\ b^{2}<4 M N \\ b^{2} \equiv 4 M N(\bmod p)}} s_{0}([M, b, N]),
$$

where $s_{0}$ is given by Theorem 2. If $M N$ is not a square (so that $T_{M}, T_{N}$ have no common components), we write $\left(T_{M} T_{N}\right)_{X}=\left(T_{M} T_{N}\right)_{X}^{\mathrm{tr}}$. If one of the curves is compact in $X$, then $\left(T_{M} T_{N}\right)_{X}$ is the intersection number in the sense of homology theory.

Theorem 3. Let $M, N$ be positive integers, $v_{p}(N) \leqq v_{p}(M)\left(v_{p}\right.$ denotes the exponent of $p$ ). Then the number of transversal intersections of the curves $T_{M}$ and $T_{N}$ on the Hilbert modular surface $X$ is given by

$$
\left(T_{M} T_{N}\right)_{X}^{\mathrm{tr}}=\frac{1}{2} \sum_{d \mid(M, N)}\left(d \chi_{p}(d)+d \chi_{p}\left(\frac{N}{d}\right)\right) H_{p}^{0}\left(M N / d^{2}\right),
$$

where

$$
H_{p}^{0}(N)=\sum_{\substack{x \in \mathbb{Z} \\ x^{2}<4 N \\ x^{2} \equiv 4 N(\bmod p)}} H\left(\frac{4 N-x^{2}}{p}\right),
$$

$H(n)$ being the class-number function defined in (15). 
Proof. By (35) and Theorem 2,

$$
\begin{aligned}
2\left(T_{M} T_{N}\right)_{X}^{\mathrm{tr}}= & \sum_{\substack{b^{2}<4 M N \\
b^{2} \equiv 4 M N(\bmod p)}} \sum_{d \mid(M, b, N)} \chi_{p}(d) d H\left(\frac{4 M N-b^{2}}{p d^{2}}\right) \\
& +\sum_{\substack{b^{2}<4 M N \\
b^{2} \equiv 4 M N(\bmod p)}} \chi_{p}\left(\frac{[M, b, N]}{(M, b, N)}\right) \sum_{d \mid(M, b, N)} \chi_{p}\left(\frac{(M, b, N)}{d}\right) d H\left(\frac{4 M N-b^{2}}{p d^{2}}\right) .
\end{aligned}
$$

In the first sum, we write $b=d x$ and may assume that $x^{2} \equiv 4 M N / d^{2}(\bmod p)$ since the only terms contributing are those with $p \nmid d$. Thus the first sum equals

$$
\sum_{d \mid(M, N)} \chi_{p}(d) d \sum_{\substack{x^{2}<4 M N / d^{2} \\ x^{2} \triangleq 4 M N / d^{2}(\bmod p)}} H\left(\left(4 M N / d^{2}-x^{2}\right) / p\right)=\sum_{d \mid(M, N)} \chi_{p}(d) d H_{p}^{0}\left(M N / d^{2}\right) .
$$

Similarly, in the second sum in the formula for $2\left(T_{M} T_{N}\right)_{X}^{\mathrm{tr}}$ we only have to sum over those $b$ for which $\left(4 M N-b^{2}\right) /(M, b, N)^{2} \equiv 0(\bmod p)$. Then $N /(M, b, N) \neq 0(\bmod p)$ and hence

$$
\chi_{p}\left(\frac{[M, b, N]}{(M, b, N)}\right)=\chi_{p}\left(\frac{N}{(M, b, N)}\right) .
$$

Therefore

$$
\chi_{p}\left(\frac{[M, b, N]}{(M, b, N)}\right) \chi_{p}\left(\frac{(M, b, N)}{d}\right)=\chi_{p}\left(\frac{N}{d}\right)
$$

and we see by the same calculation as before that the second sum equals

$$
\sum_{d \mid(M, N)} d \chi_{p}\left(\frac{N}{d}\right) H_{p}^{0}\left(\frac{M N}{d^{2}}\right) .
$$

This completes the proof of the theorem.

\subsection{Contributions from the Cusps}

As before, $X$ denotes the Hilbert modular surface $\mathfrak{S}^{2} / S L_{2}(\mathcal{O})$ for a fixed prime $p \equiv 1(\bmod 4)$. We consider the compact surface $\tilde{X}$ obtained by adding to $X$ the "cusps" and resolving the singularities thus created. Then $\tilde{X}=X \cup \bigcup_{k} S_{k}$, where each $S_{k}$ is a rational curve and the $S_{k}$ are arranged in finitely many cycles corresponding to the $h(p)$ different cusps $([4,6,7])$. The index $k$ runs through a finite indexing set $I$ which is this union of cycles. To each $k$ corresponds a reduced quadratic irrationality

$$
w_{k}=\frac{M_{k}+\sqrt{p}}{2 N_{k}}, \quad 0<w_{k}^{\prime}<1<w_{k},
$$

where $M_{k}$ and $N_{k}$ are natural numbers, and these are all reduced quadratic irrationalities of discriminant $p$. Each $w_{k}$ determines a primitive ideal $\mathfrak{a}_{k}$ (i.e. one not divisible by any natural number $>1$ ) with $\mathfrak{a}_{k}^{-1}=\mathbb{Z} w_{k}+\mathbb{Z}, \mathrm{N}\left(\mathfrak{a}_{k}\right)=N_{k}$ 
(see [4], 4.1). The ideal $\mathfrak{a}_{k}$ has characteristic $(k \mid 0,1)$ in the terminology of [4]. We have

$$
\mathfrak{a}_{k}^{\prime}=\left(N_{k}, \frac{M_{k}+\sqrt{p}}{2}\right)=\mathbb{Z} N_{k}+\mathbb{Z} \frac{M_{k}+\sqrt{p}}{2} .
$$

The integers operate on the indexing set. The result of the operation of $n \in \mathbb{Z}$ on $k \in I$ will be denoted by $k+n$. Thus $w_{k+1}$, for example, is defined, and in fact

$$
w_{k}=b_{k}-\frac{1}{w_{k+1}} \quad \text { with } b_{k} \in \mathbb{Z}, b_{k}=\left[w_{k}\right]+1 \geqq 2,
$$

and this leads to the continued fraction for $w_{k}$. The quadratic form associated to the ideal $\mathfrak{a}_{k}$ is

$$
(x, y) \mapsto \frac{1}{\mathrm{~N}\left(\mathfrak{a}_{k}\right)} \mathrm{N}\left(x N_{k}+y \frac{M_{k}-\sqrt{p}}{2}\right)=x^{2} N_{k}+x y M_{k}+y^{2} N_{k-1} .
$$

The intersection matrix $S_{k} \circ S_{l}(k, l \in I)$ has the form (at least in the case when each cycle has length $>2$ )

$$
S_{k} \circ S_{l}=\left\{\begin{array}{cl}
1 & \text { if } k=l \pm 1 \\
-b_{k} & \text { if } k=l \\
0 & \text { otherwise }
\end{array}\right.
$$

The curve $T_{N}$ defined in $X$ can be extended to a curve in $\tilde{X}$, also denoted by $T_{N}$. It meets each curve $S_{k}$ of the resolution with some multiplicity $T_{N} \circ S_{k}$. Since $\operatorname{det}\left(S_{k} \circ S_{l}\right) \neq 0$, we can introduce a divisor (with rational coefficients)

$$
T_{N}^{c}=T_{N}+\sum_{k} \alpha(N, k) S_{k}
$$

such that $T_{N}^{c} \circ S_{j}=0$ for all $j$. In order to calculate the rational coefficients $\alpha(N, k)$ we have to invert the intersection matrix $\left(S_{k} \circ S_{l}\right)$. First we introduce the following notation. Let $a$ be an ideal in $\mathcal{O}$. We put

$$
f(\mathfrak{a})=\frac{1}{\sqrt{p}} \sum_{\substack{(\lambda)=\mathfrak{a} \\ \lambda \gg 0}} \min \left(\lambda, \lambda^{\prime}\right) .
$$

Thus $f(\mathfrak{a})=0$ if $\mathfrak{a}$ is not principal. For $\mathfrak{a}$ principal, $\mathfrak{a}=(\mu)$, the sum in (36) is infinite but converges. Let $\varepsilon$ be the generator of the infinite cyclic group $U^{+}$of totally positive units of $\mathcal{O}$ which satisfies $\varepsilon<1$ and $\varepsilon^{\prime}>1$ and suppose $\mu \gg 0$ and

$$
\varepsilon^{2} \leqq \mu / \mu^{\prime} \leqq 1
$$

Then, writing $f(\mu)$ instead of $f((\mu))$, we find

$$
f(\mu)=\frac{1}{\sqrt{p}}\left(\sum_{j=0}^{\infty} \mu \varepsilon^{j}+\sum_{j=1}^{\infty} \mu^{\prime} \varepsilon^{j}\right)=\frac{1}{\sqrt{p}}\left(\frac{\mu}{1-\varepsilon}+\frac{\mu^{\prime} \varepsilon}{1-\varepsilon}\right)=\frac{1}{\operatorname{Tr} \varepsilon_{0}} \operatorname{Tr}\left(\frac{\mu \varepsilon_{0}^{\prime}}{\sqrt{p}}\right),
$$

where $\varepsilon_{0}$ is the unit (determined up to sign) satisfying $\varepsilon_{0}^{2}=\varepsilon$. After multiplying any totally positive number $\mu \in \mathcal{O}$ with a suitable power of $\varepsilon$ it will satisfy (37). 
Therefore $f(\mathfrak{a})$ is always a rational number such that $\operatorname{Tr}\left(\varepsilon_{0}\right) f(\mathfrak{a})$ is an integer. We put $g(\mu)=\left(\operatorname{Tr} \varepsilon_{0}\right)^{-1} \operatorname{Tr}\left(\mu \varepsilon_{0}^{\prime} / \sqrt{p}\right)$ and have $f(\mu)=g(\mu)$ if (37) is satisfied. We check easily:

$$
\text { if } 1 \leqq \mu / \mu^{\prime} \leqq \varepsilon^{-2}, \quad \text { then } f(\mu)=g(\mu)-\operatorname{Tr}(\mu / \sqrt{p}) \text {. }
$$

Proposition 4. The inverse matrix of the intersection matrix $\left(S_{k} \circ S_{l}\right)$ is the matrix $\left(-f\left(\mathfrak{a}_{k} \mathfrak{a}_{l}^{\prime}\right)\right)$.

Proof. We first observe that $f\left(\mathfrak{a}_{k} \mathfrak{a}_{l}^{\prime}\right)=0$ if $\mathfrak{a}_{k}, \mathfrak{a}_{l}$ are not in the same ideal class. Therefore the matrix $\left(-f\left(\mathfrak{a}_{k} \mathfrak{a}_{l}^{\prime}\right)\right)$ decomposes into blocks corresponding to the various cycles. Obviously the matrix $\left(S_{k} S_{l}\right)$ has the same property. Thus we may consider just one cycle. We shall use without further comment the notation of [4] 2.3. The ideals of the cycle are indexed by a number $k \in \mathbb{Z} / r \mathbb{Z}$, where $r$ is the length of the cycle. The indexing can be chosen in such a way that any given ideal $a_{l}$ becomes $a_{0}$. Therefore we only have to check that

$$
\begin{aligned}
& -f\left(\mathfrak{a}_{-1} \mathfrak{a}_{0}^{\prime}\right)+b_{0} f\left(\mathfrak{a}_{0} \mathfrak{a}_{0}^{\prime}\right)-f\left(\mathfrak{a}_{1} \mathfrak{a}_{0}^{\prime}\right)=1, \\
& -f\left(\mathfrak{a}_{k-1} \mathfrak{a}_{0}^{\prime}\right)+b_{k} f\left(\mathfrak{a}_{k} \mathfrak{a}_{0}^{\prime}\right)-f\left(\mathfrak{a}_{k+1} \mathfrak{a}_{0}^{\prime}\right)=0 \quad \text { for } k \neq 0
\end{aligned}
$$

(the matrix $\left(S_{k} S_{l}\right)$ was given above only for $r>2$, but these are the equations to be checked also if $r=1$ or 2 ; cf. [4] 2.4). The module $M=\mathfrak{a}_{0}^{-1}$ admits bases $\left(A_{k-1}, A_{k}\right)(k \in \mathbb{Z})$ which are given by two consecutive points of the support polygon which bounds the convex hull of $M \cap\left(\mathbb{R}_{+}\right)^{2}$, where $M$ is embedded in $\mathbb{R}^{2}$ in the usual way. We have

$$
-A_{k-1}+b_{k} A_{k}-A_{k+1}=0 .
$$

The $A_{l}$ are totally positive numbers which are monotonely decreasing $\left(A_{-1}=w_{0}\right.$, $A_{0}=1,0<A_{l+1}<A_{l}$ ) whereas the $A_{l}^{\prime}$ are increasing. We have $A_{r}=\varepsilon$ and $A_{k+r}=A_{k} \varepsilon$. In the following equations $w_{k}, \mathfrak{a}_{k}$ depend only on $k(\bmod r)$ whereas $A_{k}$ depends on $k \in \mathbb{Z}$. We have

$$
\begin{aligned}
& w_{k} A_{k}=A_{k-1}, \quad a_{k}^{-1}=A_{k}^{-1} M, \quad \mathfrak{a}_{k}=A_{k} \mathfrak{a}_{0}, \\
& \mathfrak{a}_{k} \mathfrak{a}_{l}^{\prime}=\left(A_{k} A_{l}^{\prime} \mathrm{N}\left(\mathrm{a}_{0}\right)\right)=\left(A_{k} A_{l}^{\prime} N_{0}\right) .
\end{aligned}
$$

Since $N_{0}=\mathrm{N}\left(\mathrm{a}_{0}\right)=\operatorname{Tr}\left(w_{0} / \sqrt{p}\right)^{-1}$ we have to prove

$$
\begin{aligned}
& -f\left(A_{-1}\right)+b_{0} f\left(A_{0}\right)-f\left(A_{1}\right)=\operatorname{Tr}\left(w_{0} / \sqrt{p}\right), \\
& -f\left(A_{k-1}\right)+b_{k} f\left(A_{k}\right)-f\left(A_{k+1}\right)=0 \quad \text { for } k=1, \ldots, r-1 .
\end{aligned}
$$

Since $A_{r}=\varepsilon$, the $A_{l}$ with $0 \leqq l \leqq r$ satisfy (37). Therefore $f=g$ in the second equation of (39), and the equation holds because $g$ is a linear function. On the other hand,

$$
1<w_{0} / w_{0}^{\prime}=A_{-1} / A_{-1}^{\prime} \leqq A_{-r} / A_{-r}^{\prime}=\varepsilon^{-2},
$$

so (38) applies to $A_{-1}$ and the first equation of (39) also follows from the linearity of $g$. This finishes the proof.

The coefficient $\alpha(N, k)$ of $S_{k}$ in the formula for $T_{N}^{c}$ can now be written down using the symmetric matrix $\left(f\left(\mathfrak{a}_{k} \mathfrak{a}_{l}^{\prime}\right)\right)$. We have

$$
\alpha(N, k)=\sum_{l} f\left(\mathfrak{a}_{k} \mathfrak{a}_{l}^{\prime}\right)\left(S_{l} T_{N}\right)
$$


and for the intersection numbers in the sense of rational homology

$$
\left(T_{M} T_{N}^{c}\right)_{\tilde{X}}=\left(T_{M}^{c} T_{N}\right)_{\tilde{X}}=\left(T_{M}^{c} T_{N}^{c}\right)_{\tilde{X}}=\left(T_{M} T_{N}\right)_{\tilde{X}}+\sum_{k, l} f\left(\mathfrak{a}_{k} \mathfrak{a}_{l}^{\prime}\right)\left(S_{k} T_{M}\right)\left(S_{l} T_{N}\right) .
$$

If $M N$ is not a square, then $T_{M}, T_{N}$ have no common components. The two curves meet in finitely many points of the compact surface $\tilde{X}$. Therefore $\left(T_{M} T_{N}\right)_{\tilde{X}}$ can be broken up into the intersection number $\left(T_{M} T_{N}\right)_{X}$ calculated in 1.3 and the sum of the intersection multiplicities of $T_{M}$ and $T_{N}$ in points of $\bigcup S_{k}=\tilde{X}-X$. The latter sum will be denoted by $\left(T_{M} T_{N}\right)_{\tilde{X}-X}$. We put

$$
\left(T_{M} T_{N}\right)_{\infty}=\left(T_{M} T_{N}\right)_{\bar{X}-X}+\sum_{k, l} f\left(\mathfrak{a}_{k} \mathfrak{a}_{l}^{\prime}\right)\left(S_{k} T_{M}\right)\left(S_{l} T_{N}\right)
$$

and have

$$
T_{M} T_{N}^{c}=\left(T_{M} T_{N}\right)_{X}+\left(T_{M} T_{N}\right)_{\infty} .
$$

For given $M$ the intersection of $T_{M}$ with a small neighbourhood of $\bigcup S_{k}$ is the union of all the curves

$$
u_{k}^{q}=v_{k}^{p}, \quad p \geqq 0, \quad q \geqq 0, \quad p^{2} N_{k-1}+p q M_{k}+q^{2} N_{k}=M,
$$

where $\left(u_{k}, v_{k}\right)$ is the local coordinate system in which $S_{k}$ is given by $v_{k}=0$ and $S_{k-1}$ by $u_{k}=0$. The "characteristic" $(k \mid p, q)$ determines an ideal $\mathfrak{a}$ with $\mathrm{N}(\mathfrak{a})=M$ and in this way the curves given in (42) correspond bijectively to the ideals $a$ in $\mathcal{O}$ with $\mathrm{N}(\mathfrak{a})=M$. (See [4] 4.1 and [7] 3.3.) Contrary to [4] we do not assume $(p, q)=1$. The ideal $a$ need not be primitive. The ideal $a /(p, q)$ is primitive.

In the same way, $T_{N}$ near $\bigcup S_{k}$ is just the union of all curves

$$
u_{l}^{t}=v_{l}^{s}, \quad s \geqq 0, \quad t \geqq 0, \quad s^{2} N_{l-1}+s t M_{l}+t^{2} N_{l}=N,
$$

and these correspond bijectively to the ideals $\mathrm{b}$ with $\mathrm{N}(\mathrm{b})=N$. The curve $u_{k}^{q}=v_{k}^{p}$ intersects $S_{k}$ with multiplicity $q$ and $S_{k-1}$ with multiplicity $p$, and similarly the curve $u_{l}^{t}=v_{l}^{s}$ intersects $S_{l}$ with multiplicity $t$ and $S_{l-1}$ with multiplicity $s$; other intersections with curves $S_{j}$ do not occur. Similarly, in a sufficiently small neighbourhood of $\bigcup S_{j}$ the curves (42) and (43) intersect only if $k=l$, their intersection point then being the origin of the $k$-th coordinate system $\left(u_{k}, v_{k}\right)$. Since the intersection number of the affine curves $u^{q}-v^{p}=0, u^{t}-v^{s}=0$ at the origin (for $p t-q s \neq 0)$ is $\min (p t, q s)$, the intersection number of the curves (42) and (43) near $\bigcup S_{j}$ is $\delta_{k l} \min (p t, q s)$.

Proposition 5. Assume that $M N$ is not a square. Then

$$
\left(T_{M} T_{N}\right)_{\infty}=\sum_{\substack{\mathrm{N}(a)=M \\ \mathrm{~N}(\mathrm{~b})=N}} f\left(\mathfrak{a}^{\prime}\right) .
$$

(The sum is taken over all ideals $\mathfrak{a}, \mathfrak{b}$ in $\mathcal{O}$ with $\mathrm{N}(\mathfrak{a})=M$ and $\mathrm{N}(\mathfrak{b})=N$ and is of course finite. It can also be written as $\sum f(\mathfrak{a} b)$.)

Proof. The ideals $\mathfrak{a}, \mathfrak{b}$ correspond to curves (42) and (43), respectively. Each pair of such curves contributes to $\left(T_{M} T_{N}\right)_{\infty}$ as defined by (41) the following expression:

$$
\delta_{k l} \min (p t, q s)+f\left(a_{k-1} \mathfrak{a}_{l-1}^{\prime}\right) p s+f\left(\mathfrak{a}_{k-1} \mathfrak{a}_{l}^{\prime}\right) p t+f\left(\mathfrak{a}_{k} \mathfrak{a}_{l-1}^{\prime}\right) q s+f\left(\mathfrak{a}_{k} \mathfrak{a}_{l}^{\prime}\right) q t,
$$


and we have to show that this equals $f\left(\mathfrak{a} \mathfrak{b}^{\prime}\right)$. Interchanging the roles of $T_{M}$ and $T_{N}$ if necessary, we may assume that $p t \leqq q s$ if $k=l$.

As in the proof of the preceding proposition, we may suppose that $k, l$ belong to the same cycle and (after re-indexing) that $l=0$ and $0 \leqq k \leqq r-1$, where $r$ is the length of the cycle. Then $\mathfrak{a}_{k}=A_{k} \mathfrak{a}_{0}, \mathfrak{a}_{-1}=A_{-1} \mathfrak{a}_{0}=w_{0} \mathfrak{a}_{0}$. By [4] 4.1(6), (8),

$$
\begin{array}{ll}
\mathfrak{a}=\lambda \mathrm{a}_{0} & \text { with } \lambda=p A_{k-1}+q A_{k}, \\
\mathfrak{b}=\mu \mathrm{a}_{0} & \text { with } \mu=s A_{-1}+t A_{0}=s w_{0}+t .
\end{array}
$$

If $k \geqq 1$ we have to show

$$
f\left(A_{k-1} w_{0}^{\prime}\right) p s+f\left(A_{k-1}\right) p t+f\left(A_{k} w_{0}^{\prime}\right) q s+f\left(A_{k}\right) q t=f\left(\lambda \mu^{\prime}\right) .
$$

Since the sequence $\left\{A_{j}\right\}$ is monotonely decreasing and $\left\{A_{j}^{\prime}\right\}$ is monotonely increasing, and $A_{j+\mathrm{r}}=A_{j} \varepsilon$ for all $j$, we have

$$
1=A_{0} / A_{0}^{\prime} \geqq A_{k-1} / A_{k-1}^{\prime} \geqq A_{k} / A_{k}^{\prime} \geqq A_{r} / A_{r}^{\prime}=\varepsilon^{2}
$$

and

$$
1 \geqq A_{k-1} A_{-1}^{\prime} / A_{k-1}^{\prime} A_{-1} \geqq A_{k} A_{-1}^{\prime} / A_{k}^{\prime} A_{-1} \geqq A_{r-1} A_{-1}^{\prime} / A_{r-1}^{\prime} A_{-1}=\varepsilon^{2},
$$

so all four numbers $A_{k-1} w_{0}^{\prime}, A_{k-1}, A_{k} w_{0}^{\prime}, A_{k}$ satisfy (37). The number $\lambda \mu^{\prime}$ is a linear combination of these four numbers with nonnegative coefficients, so it also satisfies (37). Thus for all five of these numbers $f$ can be replaced by $g$ and (44) follows from the linearity of $g$. If $k=0, p t \leqq q s$, we must show

$$
\frac{p t}{\mathrm{~N}\left(\mathfrak{a}_{0}\right)}+f\left(w_{0} w_{0}^{\prime}\right) p s+f\left(w_{0}\right) p t+f\left(w_{0}^{\prime}\right) q s+f(1) q t=f\left(\lambda \mu^{\prime}\right) .
$$

The numbers $w_{0} w_{0}^{\prime}, w_{0}^{\prime}$ and 1 all satisfy (37), and therefore

$$
\lambda \mu^{\prime}=\left(p w_{0}+q\right)\left(s w_{0}^{\prime}+t\right)=\left(p s w_{0} w_{0}^{\prime}+p t\left(w_{0}+w_{0}^{\prime}\right)+q t\right)+(q s-p t) w_{0}^{\prime}
$$

also does, so for these four numbers $f=g$; on the other hand, $w_{0}$ satisfies the assumption of (38) and therefore

$$
f\left(w_{0}\right)=g\left(w_{0}\right)-\operatorname{Tr}\left(w_{0} / \sqrt{p}\right)=g\left(w_{0}\right)-\frac{1}{\mathrm{~N}\left(\mathfrak{a}_{0}\right)},
$$

so (45) also follows from the linearity of $g$. This completes the proof.

\subsection{Self-Intersections}

The curve $T_{N}$ in $X=\mathfrak{S}^{2} / S L_{2}(\mathcal{O})$ is a union of irreducible curves each of which has a non-singular model $\mathfrak{H} / \Gamma$, where $\Gamma$ is a discrete subgroup of $S L_{2}(\mathbb{R})$. In $\mathfrak{H}$ we have the $S L_{2}(\mathbb{R})$-invariant volume form which we normalize in such a way that the volume of the fundamental domain of $S L_{2}(\mathbb{Z})$ becomes $-\frac{1}{6}$ (see [4] $\$ 1$ ). The above mentioned curves $\mathfrak{S} / \Gamma$ all have finite volumes and thus the volume of $T_{N}$ is well-defined. We have

$$
\operatorname{vol}\left(T_{N}\right)=-\frac{1}{12} \sum_{d \mid N}\left(\chi_{p}(d)+\chi_{p}(N / d)\right) d .
$$


This result was mentioned in [5] (15) for $p \nmid N$. It is a consequence of a volume calculation due to Eichler [1]. The proof of (46) will be contained in a forthcoming Bonn dissertation by H.G. Franke.

For positive integers $M, N$ we define

$$
\left(T_{M} T_{N}\right)_{X}=\left(T_{M} T_{N}\right)_{X}^{\mathrm{tr}}+\operatorname{vol}\left(\left(T_{M} \cap T_{N}\right)^{1}\right),
$$

where $\left(T_{M} \cap T_{N}\right)^{1}$ is the one-dimensional part of the intersection of $T_{M}$ and $T_{N}$, which is empty if $M N$ is not a square and equals $T_{(M, N)}$ if $M N$ is a square. For $\left(T_{M} T_{N}\right)_{X}^{\text {tr }}$ see 1.3 .

Let us now consider the case that $M N$ is a square. Assume $v_{p}(N) \leqq v_{p}(M)$. Then $v_{p}((M, N))=v_{p}(N)$ and $N /(M, N)$ is a square prime to $p$. Hence $\chi_{p}(N / d)=$ $\chi_{p}((M, N) / d)$ and by $(46)$

$$
\operatorname{vol}\left(T_{(M, N)}\right)=\sum_{d \mid(M, N)}\left(d \chi_{p}(d)+d \chi_{p}(N / d)\right) H(0),
$$

where $H(0)=-1 / 12$ by definition. In 1.4 (Theorem 3) we defined a function $H_{p}^{0}$. It is more natural to introduce the function

$$
H_{p}(n)=\sum_{\substack{x \in \mathbb{Z} \\ x^{2} \leq n \\ x^{2} \equiv 4 n(\bmod p)}} H\left(\frac{4 n-x^{2}}{p}\right) .
$$

If $n$ is not a square, then $H_{p}(n)=H_{p}^{0}(n)$. If $n>0$ is a square, then $H_{p}(n)-H_{p}^{0}(n)=$ $2 H(0)$. Therefore by (47), (48) and Theorem 3

$$
\left(T_{M} T_{N}\right)_{X}=\frac{1}{2} \sum_{d \mid(M, N)}\left(d \chi_{p}(d)+d \chi_{p}(N / d)\right) H_{p}\left(M N / d^{2}\right) \quad \text { if } v_{p}(N) \leqq v_{p}(M),
$$

and this formula holds for all values of $M$ and $N$.

If $M N$ is not a square we have

$$
\left(T_{M} T_{N}^{c}\right)_{\tilde{X}}=\left(T_{M} T_{N}\right)_{X}+\left(T_{M} T_{N}\right)_{\infty}
$$

with $\left(T_{M} T_{N}\right)_{\infty}$ given by Proposition 5. We want to show that the same formula holds if $M N$ is a square.

Proposition 6. For the curves $T_{N}, T_{N}$ of the Hilbert modular surface $\tilde{X}$ we have

$$
T_{M} T_{N}^{c}=T_{M}^{c} T_{N}=\left(T_{M} T_{N}\right)_{X}+\left(T_{M} T_{N}\right)_{\infty}
$$

with

$$
\left(T_{M} T_{N}\right)_{\infty}=\sum f\left(\mathbf{a b}^{\prime}\right),
$$

where the sum is taken over all ideals $\mathfrak{a}, \mathfrak{b}$ in $\mathcal{O}$ with $\mathrm{N}(\mathfrak{a})=M$ and $\mathrm{N}(\mathfrak{b})=N$.

We have to calculate $\left(T_{M} T_{N}\right)_{\tilde{X}}$ and this involves $\left(T_{(M, N)} T_{(M, N)}\right)_{\tilde{X}}$ if $M N$ is a square. Self-intersection numbers can be evaluated by the adjunction formula (compare [4] 0.6). Therefore the proof of Proposition 6 will be preceded by a discussion of the adjunction formula for curves in complex surfaces (Plücker formula) and its generalization to surfaces with isolated quotient singularities.

Let $D$ be a compact curve (not necessarily irreducible) on the non-singular complex surface $Y$. Then the Euler number of the non-singular model $\tilde{D}$ of $D$ 
is given by the formula

$$
e(\tilde{D})=c_{1}[D]-D D+\sum_{x \in Y} \mu_{x}(D),
$$

where $c_{1}$ is the first Chern class of $Y$ and the Plücker number $\mu_{x}(D)$ is a nonnegative even integer depending only on the germ of $D$ in $x$ which is positive if and only if $x$ is a singular point of $D$. For example, if $r$ non-singular branches of $D$ intersect pairwise transversally in $x$, then $\mu_{x}(D)=r(r-1)$. Now we consider a complex surface $Y$ which is allowed to have isolated quotient singularities. For each $x \in Y$ we can choose a sufficiently small neighbourhood $U_{x}$ of $x$ in $Y$, an open ball $V_{x}$ around the origin of $\mathbb{C}^{2}$ with a linear action of a finite group $G_{x}$ on $V_{x}$ which is free on $V_{x}-\{0\}$, and a map $\pi: V_{x} \rightarrow U_{x}$ with $\pi^{-1}(x)=0$ which induces an isomorphism $V_{x} / G_{x} \rightarrow U_{x}$. If $D$ is a compact curve in $Y$ passing through $x$, we consider $\pi^{-1}\left(D \cap U_{x}\right)$ and define $\mu_{x}(D)=\frac{1}{\left|G_{x}\right|} \mu_{0}\left(\pi^{-1}\left(D \cap U_{x}\right)\right)$.

For every irreducible branch $D_{x, j}$ of $D$ in $x$ the inverse image under $\pi$ consists of $v_{x, j}$ irreducible branches, each covering $D_{x, j}$ with multiplicity $r_{x, j}=\left|G_{x}\right| / v_{x, j}$. We define a modified Euler number $e^{\prime}(\tilde{D})$ by

$$
e^{\prime}(\tilde{D})=e(\tilde{D})-\sum_{x, j} \frac{r_{x, j}-1}{r_{x, j}},
$$

where $\tilde{D}$ is the non-singular model of $D$ and the sum extends over all irreducible branches of $D$ in quotient singularities of $Y$. Let $Y^{\prime}$ be the non-singular surface obtained from $Y$ by removing the quotient singularities. Then $H^{2}\left(Y^{\prime} ; \mathbb{Q}\right) \cong$ $H^{2}(Y ; \mathbb{Q})$ (by Mayer-Vietoris), so the first Chern class of $Y^{\prime}$ defines a class $c_{1} \in H^{2}(Y ; \mathbb{Q})$ which we will call the first Chern class of $Y$.

Lemma 4. Let $Y$ be a complex surface with isolated quotient singularities, $c_{1}$ the first Chern class of $Y$, and $D$ a compact curve on $Y$. Then

$$
e^{\prime}(\tilde{D})=c_{1}[D]-D D+\sum_{x \in Y} \mu_{x}(D) \text {. }
$$

We omit the proof of this lemma. It uses the fact that for a non-singular point $x$ the number $\mu_{x}(D)$ equals the Milnor number of $D$ in $x$ (which Milnor introduced in his study of isolated singularities of hypersurfaces) plus the number of branches of $D$ in $x$ minus one.

Let $D_{1}, D_{2}$ be compact curves on $Y$ and $D$ the one-dimensional part of $D_{1} \cap D_{2}$. Write $D_{1}^{\prime}=D_{1}-D, D_{2}^{\prime}=D_{2}-D$. Then by Lemma 4

$$
D_{1} D_{2}=D\left(D_{1}^{\prime}+D_{2}^{\prime}\right)+D_{1}^{\prime} D_{2}^{\prime}+\sum_{x \in Y} \mu_{x}(D)+c_{1}[D]-e^{\prime}(\tilde{D}) \text {. }
$$

In other words, we have

where

$$
D_{1} D_{2}=\sum_{x \in \boldsymbol{Y}} \mu_{x}\left(D_{1}, D_{2}\right)+c_{1}[D]-e^{\prime}(\tilde{D})
$$

$$
\mu_{x}\left(D_{1}, D_{2}\right)=\left(D\left(D_{1}^{\prime}+D_{2}^{\prime}\right)\right)_{x}+\left(D_{1}^{\prime} D_{2}^{\prime}\right)_{x}+\mu_{x}(D),
$$

$\left(D\left(D_{1}^{\prime}+D_{2}^{\prime}\right)\right)_{x}$ and $\left(D_{1}^{\prime} D_{2}^{\prime}\right)_{x}$ being local intersection numbers. 
We now come to the proof of Proposition 6. We assume $M N$ is a square. For $z \in X$ with $\left|S L_{2}(\mathcal{O})_{z}\right|=v_{3}$ and such that $r$ branches of the inverse image of $T_{(M, N)}$ in $\mathfrak{S}^{2}$ pass through a representative $z$ of $\mathfrak{z}$ in $\mathfrak{S}^{2}$ we have

$$
\mu_{3}\left(T_{(M, N)}\right)=\frac{2 r(r-1)}{v_{3}}=\left(T_{(M, N)} T_{(M, N)}\right)_{3}^{\mathrm{tr}},
$$

and therefore

$$
\mu_{3}\left(T_{M}, T_{N}\right)=\left(T_{M} T_{N}\right)_{3}^{\mathrm{ir}}
$$

As explained in 1.4, the curve $T_{(M, N)}$ near $\bigcup S_{j}$ is given by local equations $u_{k}^{b}-v_{k}^{a}=0$ corresponding to the ideals $\mathrm{c}$ in $\mathcal{O}$ with $\mathrm{N}(\mathrm{c})=(M, N)$, the characteristic of $c$ being $(k \mid a, b)$. Locally such a curve has $(a, b)$ branches and hence the number of cusps of $T_{(M, N)}$ in the sense of the one-dimensional theory is $\sum_{c}(a, b)$, where the sum extends over all ideals $c$ with norm $(M, N)$. By [4] $4.3(21)$ we have for the non-singular model $\tilde{T}_{(M, N)}$ of $T_{(M, N)}$

$$
e^{\prime}\left(\tilde{T}_{(M, N)}\right)=\operatorname{vol}\left(T_{(M, N)}\right)+\sum_{c}(a, b) .
$$

It follows from [4] $4.3(19)$ that

$$
\tilde{c}_{1}\left[T_{(M, N)}\right]=2 \operatorname{vol}\left(T_{(M, N)}\right)+T_{(M, N)} \cdot\left(\sum S_{k}\right)=2 \operatorname{vol}\left(T_{(M, N)}\right)+\sum_{c}(a+b),
$$

where $\tilde{c}_{1} \in H^{2}(\tilde{X} ; \mathbb{Q})$ is the first Chern class of $\tilde{X}$. By $(47),(52),(54),(55)$ and (56) we obtain

$$
\left(T_{M} T_{N}\right)_{\tilde{X}}=\left(T_{M} T_{N}\right)_{X}+\sum_{c}(a+b-(a, b))+\sum_{x \in X} \mu_{x}\left(T_{M}, T_{N}\right),
$$

where the second sum runs over $x \in T_{M} \cap T_{N} \cap \bigcup S_{j}$. The intersection of $T_{M}$ or $T_{N}$, respectively, with a small neighbourhood of $\bigcup S_{j}$ is described in (42) and (43). We must calculate $\mu_{x}(A, B)$ where $A$ is the curve $u_{k}^{q}=v_{k}^{p}, B$ the curve $u_{k}^{t}=v_{k}^{s}$ and $x$ the origin of the coordinate system $\left(u_{k}, v_{k}\right)$. If $p t-q s \neq 0$, then $A$ and $B$ have no common branch in $x$ and $\mu_{x}(A, B)$ is just the local intersection number $\min (p t, q s)$ of $A$ and $B$ in $x$. If $p t-q s=0$, then we write $\frac{p}{s}=\frac{q}{t}=\frac{\alpha}{\beta}$ with $(\alpha, \beta)=1$ and put $\frac{p}{\alpha}=\frac{s}{\beta}=a$ and $\frac{q}{\alpha}=\frac{t}{\beta}=b$. Then $A \cap B$ is the curve $u_{k}^{b}=v_{k}^{a}$ with

$$
\mu_{x}(A \cap B)=(a-1)(b-1)+(a, b)-1=a b-(a+b)+(a, b)
$$

$((a-1)(b-1)$ is the Milnor number, $(a, b)$ the number of branches). The curve $A$ is the union of $A \cap B$ and the curves $u_{k}^{b}=\xi v_{k}^{a}\left(\xi^{\alpha}=1, \xi \neq 1\right)$ and $B$ is the union of $A \cap B$ and the curves $u_{k}^{b}=\eta v_{k}^{a}\left(\eta^{\beta}=1, \eta \neq 1\right)$. Each pair of curves $u_{k}^{b}=\xi v_{k}^{a}$, $u_{k}^{b}=\eta v_{k}^{a}$ with $(\xi, \eta) \neq(1,1)$ has the intersection number $(a, b)$. Therefore, since $\alpha \beta a b=p t=q s$, we have $\mu_{x}(A, B)=\min (p t, q s)-(a+b)+(a, b)$. The ideal $a$ with characteristic $(k \mid p, q)$ has norm $M$, the ideal $\mathbf{b}$ with characteristic $(k \mid s, t)$ has norm $N$. Then $a / \alpha=b / \beta$ and this is an ideal $c$ with norm $(M, N)$ and characteristic $(k \mid a, b)$. We have $\alpha^{2}=M /(M, N)$ and $\beta^{2}=N /(M, N)$. Every ideal $c$ with 
$\mathrm{N}(\mathrm{c})=(M, N)$ is obtained in this way. Therefore (57) yields

$$
\left(T_{M} T_{N}\right)_{\tilde{X}}=\left(T_{M} T_{N}\right)_{X}+\sum_{\substack{\mathrm{N}(\mathbf{a})=M \\ \mathrm{~N}(\mathrm{~b})=N}} \delta_{k l} \min (p t, q s),
$$

where the sum is over all ideals $\mathfrak{a}, \mathfrak{b}$ with $\mathrm{N}(\mathfrak{a})=M, \mathrm{~N}(\mathfrak{b})=N$ and $(k \mid p, q),(l \mid s, t)$ are the characteristics of $\mathfrak{a}$ and $\mathfrak{b}$. Since

$$
T_{M} T_{N}^{c}=\left(T_{M} T_{N}\right)_{\tilde{X}}+\sum_{k, l} f\left(\mathfrak{a}_{k} \mathfrak{a}_{l}^{\prime}\right)\left(S_{k} T_{M}\right)\left(S_{l} T_{N}\right)
$$

the proof of Proposition 6 is now identical with the proof of Proposition 5. The number $\left(T_{1} T_{N}\right)_{\infty}=\sum_{N(\mathfrak{a})=N} f(\mathfrak{a})$ equals the number $I_{p}(N)$ defined in (4) of the
Introduction. We have

$$
\left(T_{M} T_{N}\right)_{\infty}=\sum_{\substack{N(a)=M \\ N(\mathfrak{b})=N}} f\left(\mathfrak{a} \mathfrak{b}^{\prime}\right)=\sum_{d \mid(M, N)} d \chi_{p}(d) I_{p}\left(M N / d^{2}\right) .
$$

This is a consequence of the following lemma applied to the function $f$.

Lemma 5. Let $g$ be a function on integral ideals of a real quadratic field $K$ of discriminant $D$ satisfying $g(d \mathfrak{a})=d^{r} g(\mathfrak{a})$ for some fixed $r$ and for all ideals $\mathfrak{a}$ and natural numbers $d$. Let $G(N)=\sum_{\mathrm{N}(\mathfrak{a})=N} g(\mathfrak{a})$. Then

$$
\sum_{\substack{\mathrm{N}(\mathfrak{a})=M \\ \mathrm{~N}(\mathfrak{b})=N}} g(\mathfrak{a} \mathfrak{b})=\sum_{d \mid(M, N)} d^{r} \chi_{D}(d) G\left(M N / d^{2}\right)
$$

Proof. We denote the left-hand side of $(60)$ by $G(M, N)$ and calculate the corresponding generating (Dirichlet) series:

$$
\begin{aligned}
\sum_{M=1}^{\infty} \sum_{N=1}^{\infty} G(M, N) M^{-s} N^{-t} & =\sum_{\mathfrak{a}, \mathfrak{b}} g(\mathfrak{a} \mathfrak{b}) \mathrm{N}(\mathfrak{a})^{-s} \mathrm{~N}(\mathfrak{b})^{-t} \\
& =\sum_{\mathfrak{c}} g(\mathfrak{c}) \sum_{\mathfrak{a} \mid \mathfrak{c}} \mathrm{N}(\mathfrak{a})^{-s} \mathrm{~N}(\mathfrak{c} / \mathfrak{a})^{-t} \\
& =\sum_{\mathfrak{c}} g(\mathfrak{c}) \sigma_{t-s}(\mathfrak{c}) \mathrm{N}(\mathfrak{c})^{-t},
\end{aligned}
$$

where $\mathfrak{a}, \mathfrak{b}, \mathfrak{c}$ run through the integral ideals of $K$ and $\sigma_{k}(\mathfrak{c})$ is defined in analogy with the standard function $\sigma_{k}(n)=\sum_{d \mid n} d^{k}(n \in \mathbb{Z}, n>0)$ as the sum of the $k$-th powers of the norms of all ideals dividing $c$. By the Lemma in $\S 3$ of [15],

$$
\sigma_{k}(\mathrm{c})=\sum_{d \mid c} d^{k} \chi_{D}(d) \sigma_{k}\left(\mathrm{~N}(\mathrm{c}) / d^{2}\right)
$$

for any $k$, where the sum is over all natural numbers $d$ dividing the ideal $c$. Hence

$$
\begin{aligned}
\sum_{M, N} G(M, N) M^{-s} N^{-t} & =\sum_{\mathfrak{c}} g(\mathfrak{c}) \mathrm{N}(\mathfrak{c})^{-t} \sum_{d \mid \mathfrak{c}} d^{t-s} \chi_{D}(d) \sigma_{t-s}\left(\mathrm{~N}(\mathfrak{c}) / d^{2}\right) \\
& =\sum_{d=1}^{\infty} \sum_{\mathfrak{a}} g(d \mathfrak{a}) \mathrm{N}(d \mathfrak{a})^{-t} d^{t-s} \chi_{D}(d) \sigma_{t-s}(\mathrm{~N}(\mathfrak{a})) \\
& =\sum_{d=1}^{\infty} \sum_{\mathfrak{a}} d^{r} g(\mathfrak{a}) d^{-2 t} \mathrm{~N}(\mathfrak{a})^{-t} d^{t-s} \chi_{D}(d) \sigma_{t-s}(\mathrm{~N}(\mathfrak{a}))
\end{aligned}
$$




$$
\begin{aligned}
& =\left(\sum_{d=1}^{\infty} \chi_{D}(d) d^{r-t-s}\right)\left(\sum_{N=1}^{\infty} G(N) \sigma_{t-s}(N) N^{-t}\right) \\
& =\left(\sum_{d=1}^{\infty} \chi_{D}(d) d^{r-t-s}\right)\left(\sum_{m=1}^{\infty} \sum_{n=1}^{\infty} G(m n) m^{-s} n^{-t}\right) \\
& =\sum_{M=1}^{\infty} \sum_{N=1}^{\infty}\left(\sum_{d \mid(M, N)} \chi_{D}(d) d^{r} G\left(M N / d^{2}\right)\right) M^{-s} N^{-t},
\end{aligned}
$$

and this proves the lemma.

We have now completed the calculation of $\left(T_{M} T_{N}^{c}\right)_{\tilde{X}}$ in all cases. Before stating the final result, we rewrite (59) in a form similar to (50):

$$
\left(T_{M} T_{N}\right)_{\infty}=\frac{1}{2} \sum_{d \mid(M, N)}\left(d \chi_{p}(d)+d \chi_{p}(N / d)\right) I_{p}\left(M N / d^{2}\right) \quad \text { if } v_{p}(N) \leqq v_{p}(M) .
$$

To see that this holds, write $N=p^{v} N_{0}, M=p^{v} M_{0}$ with $p \nmid N_{0}$. Then

$$
\begin{aligned}
& \sum_{d \mid(\boldsymbol{M}, N)} d \chi_{p}(d) I_{p}\left(M N / d^{2}\right)=\sum_{d_{0} \mid\left(M_{0}, N_{0}\right)} d_{0} \chi_{p}\left(d_{0}\right) I_{p}\left(p^{2 v} M_{0} N_{0} / d_{0}^{2}\right), \\
& \sum_{d \mid(M, N)} d \chi_{p}(N / d) I_{p}\left(M N / d^{2}\right)=\sum_{d_{0 \mid} \mid\left(M_{0}, N_{0}\right)} d_{0} p^{v} \chi_{p}\left(N_{0} / d_{0}\right) I_{p}\left(M_{0} N_{0} / d_{0}^{2}\right) .
\end{aligned}
$$

These expressions differ only by a factor $\chi_{p}\left(N_{0}\right)$, since clearly $I_{p}\left(p^{2 v} n\right)=p^{v} I_{p}(n)$ for any $n$. Thus if $\chi_{p}\left(N_{0}\right)=1,(61)$ reduces to $(59)$, while if $\chi_{p}\left(N_{0}\right)=-1$ both sides of (61) are zero (the left-hand side because $N$ is not a norm).

Summing up, we have proved:

Theorem 4. Let $M, N$ be positive integers, $v_{p}(N) \leqq v_{p}(M)$. Then the intersection number of the homology classes $T_{M}^{c}$ and $T_{N}^{c}$ on the compact surface $\tilde{X}$ is given by

$$
T_{M} T_{N}^{c}=\frac{1}{2} \sum_{d \mid(M, N)}\left(d \chi_{p}(d)+d \chi_{p}(N / d)\right)\left(H_{p}\left(M N / d^{2}\right)+I_{p}\left(M N / d^{2}\right)\right),
$$

where $H_{p}$ and $I_{p}$ are the functions defined in Equations (3) and (4) of the Introduction.

\section{Chapter 2: Modular Forms Whose Fourier Coefficients Involve Class Numbers}

Notation. We again fix a real quadratic field $K$. The discriminant of $K$ is denoted $D$; the other notations concerning $K\left(\mathcal{O}, x^{\prime}, x \gg 0, \mathrm{~N}(x), \operatorname{Tr}(x)\right)$ are the same as in Chapter 1. As before, $\mathfrak{G}$ denotes the upper half-plane; $\mathbb{R}_{+}$and $\mathbb{R}_{-}$denote the sets of real numbers $\geqq 0$ and $\leqq 0$, respectively, $\mathbb{N}$ the set of integers $\geqq 0$. For $z \in \mathbb{C}, n \in \mathbb{Z}$, we write $\mathrm{e}(z)$ for $e^{2 \pi i z}$ and $z^{n / 2}$ for $|z|^{n / 2} e^{i n \arg (z) / 2}$ with $-\pi<\arg (z) \leqq \pi$.

For $k>0$ even, $M_{k}\left(\Gamma_{0}(D), \chi_{D}\right)$ denotes the vector space of modular forms of weight $k$, level $D$ and "Nebentypus" $\chi_{D}$, i.e. of functions $f: \mathfrak{H} \rightarrow \mathbb{C}$ satisfying

$$
f\left(\frac{a z+b}{c z+d}\right)=\chi_{D}(a)(c z+d)^{k} f(z) \quad\left(z \in \mathfrak{H},\left(\begin{array}{ll}
a & b \\
c & d
\end{array}\right) \in \Gamma_{0}(D)\right)
$$

and which are holomorphic on $\mathfrak{H}$ and at the cusps of $\Gamma_{0}(D)$. The (infinitedimensional) vector space of functions $f: \mathfrak{H} \rightarrow \mathbb{C}$ satisfying (1), with no holomorphy conditions, is denoted $M_{k}^{*}\left(\Gamma_{0}(D), \chi_{D}\right)$; such functions will be called "nonanalytic modular forms" (of weight $k$, level $D$ and Nebentypus). 


\subsection{The Modular Form $\varphi_{D}(z)$}

Let $D$ be the discriminant of a real quadratic field. For $N \in \mathbb{N}$, set

$$
c(N)=H_{D}(N)+I_{D}(N),
$$

where

$$
H_{D}(N)=\sum_{\substack{t \in \mathbb{Z} \\ t^{2} \leq 4 N \\ t^{2} \equiv 4 N(\bmod D)}} H\left(\frac{4 N-t^{2}}{D}\right)
$$

$(H(n)$ being the class number function defined in 1.2) and

$$
I_{D}(N)=\frac{1}{\sqrt{D}} \sum_{\substack{\lambda \in \mathcal{O}, \lambda \geqslant 0 \\ \lambda \lambda^{\prime}=N}} \min \left(\lambda, \lambda^{\prime}\right) .
$$

We saw in Chapter 1 that, at least if $D$ is prime, $c(N)(N>0)$ represents the intersection number $T_{1} T_{N}^{c}$ on an appropriate compactification of the Hilbert modular surface $\mathfrak{S}^{2} / S L_{2}(\mathcal{O}), H_{D}(N)$ being the actual intersection number of the curves $T_{1}$ and $T_{N}$ on this surface and $I_{D}(N)$ the contribution from the cusps. For $N=0, c(N)=-1 / 12$ is half the volume of the curve $T_{1}$. The main result of this chapter is that the numbers $c(N)$ are the Fourier coefficients of a modular form in $M_{2}\left(\Gamma_{0}(D), \chi_{D}\right)$.

Theorem 1. The function

$$
\varphi_{D}(z)=\sum_{N=0}^{\infty} c(N) e^{2 \pi i N z} \quad(z \in \mathfrak{H})
$$

is a modular form of weight 2 and Nebentypus $\chi_{D}$ for $\Gamma_{0}(D)$.

This theorem is similar to various classical class number identities of Kronecker, Hurwitz and others (see bibliography) in which various expressions involving class numbers are shown to be equal to Fourier coefficients of modular forms. One such result, for example, due to Hurwitz [17], says that the expression $c(N)$ in the case $D=1$, i.e. the number

$$
H_{1}(N)+\sum_{\substack{\lambda=\left(\lambda_{1}, \lambda_{2}\right) \in \mathbb{Z} \times \mathbb{Z} \\ \lambda_{1}, \lambda_{2}>0 \\ \lambda_{1} \lambda_{2}=N}} \min \left(\lambda_{1}, \lambda_{2}\right),
$$

is equal to $2 \sigma_{1}(N)$ if $N>0$, where $\sigma_{1}(N)$ as usual denotes the sum of the positive divisors of $N$; thus $\varphi_{1}(z)$ is $-\frac{1}{12}$ times the normalized Eisenstein series

$$
E_{2}(z)=1-24 \sum_{N=1}^{\infty} \sigma_{1}(N) e^{2 \pi i N z} .
$$

(This is of course not a special case of Theorem 1.) We now describe briefly some other related results and generalizations of Theorem 1. 
For any positive even integer $k$, we set

$$
c_{k}(N)=\sum_{t} p_{k}(t, N) H\left(\frac{4 N-t^{2}}{D}\right)+\frac{1}{\sqrt{D}} \sum_{\lambda} \min \left(\lambda, \lambda^{\prime}\right)^{k-1},
$$

where the summations are the same as in the definition of $c(N)$ and $p_{k}(t, N)$ is the polynomial defined by

$$
p_{k}(t, N)=\left(\rho_{+}^{k-1}-\rho_{-}^{k-1}\right) /\left(\rho_{+}-\rho_{-}\right), \quad \rho_{ \pm}=\frac{1}{2}\left(t \pm \sqrt{t^{2}-4 N}\right) .
$$

Then the function $\varphi_{D, k}(z)=\sum c_{k}(N) e^{2 \pi i N z}$ is a modular form of weight $k$ for the group $\Gamma_{0}(D)$ and character $\chi_{D}$ and in fact a cusp form for $k>2$. If $k>2$ and $D=1$, then the Selberg-Eichler trace formula [32] tells us that

$$
c_{k}(N)=-2 \operatorname{Tr}\left(T(N), S_{k}\left(S L_{2} \mathbb{Z}\right)\right),
$$

where $T(N)$ is a Hecke operator on the space of cusp forms of weight $k$ for the full modular group, so the function $\varphi_{1, k}(z)$ is -2 times the sum of all normalized Hecke eigenfunctions in this space. The proofs of these results, as well as new proofs of the results of Cohen mentioned below and various generalizations, will be given in [36].

The other result related to Theorem 1 which we would like to discuss is due to Cohen [23]. Let $r$ be an odd positive integer, and define an arithmetical function $H(r, N)(N \in \mathbb{N})$ by $H(r, 0)=\zeta(1-2 r), H(r, N)=0$ for $N \equiv 1$ or $2(\bmod 4)$, and

$$
H(r, N)=L\left(1-r, \chi_{\Delta}\right) \sum_{d \mid f} d^{2 r-1} \prod_{p \mid d}\left(1-\chi_{\Delta}(p) p^{-r}\right)
$$

for $N>0, N \equiv 0$ or $3(\bmod 4)$, where $\Delta<0$ is the discriminant of $\mathbb{Q}(\sqrt{-N})$ and $f$ is defined by $-N=\Delta f^{2}$. This function generalizes the class number function $H(N)=H(1, N)$ (cf. Eq. (15) and Proposition 1 of 1.2). We set

$$
H_{D}(r, N)=\sum_{t} H\left(r, \frac{4 N-t^{2}}{D}\right)
$$

the summation being the same as before. Then Cohen shows that, for $r>1$, the function $\sum_{N=0}^{\infty} H_{D}(r, N) e^{2 \pi i N z}$ belongs to $M_{r+1}\left(I_{0}(D), \chi_{D}\right)$. Thus for $r>1$ no corrective term like our $\sum \min \left(\lambda, \lambda^{\prime}\right)$ is needed. As with the case $r=1$, there is a generalization in which the terms $H\left(r, \frac{4 N-t^{2}}{D}\right)$ in the sum above are weighted with a certain homogeneous polynomial in $t$ and $N$, leading to modular forms (in fact cusp forms) of higher weight. Unfortunately, Cohen's proof does not work for $r=1$, although the starting point for both proofs, as we shall see, is the same.

The basic idea is to express the numbers $H(N)$ as Fourier coefficients of a modular form of half-integral weight. This suggestion was already made by Hecke [20] as a way of explaining the classical class number relations like the above-mentioned theorem of Hurwitz concerning $H_{1}(N)$. Hecke pointed out that, by the formula of Gauss and Hermite, the number $r_{3}(N)$ of representations 
of $N$ as a sum of three squares can be expressed in terms of class numbers:

$$
r_{3}(N)= \begin{cases}12 H(N) & \text { if } N \equiv 1 \operatorname{or} 2(\bmod 4), \\ 24 H(4 N) & \text { if } N \equiv 3(\bmod 8), \\ 0 & \text { if } N \equiv 7(\bmod 8), \\ r_{3}(N / 4) & \text { if } N \equiv 0(\bmod 4) .\end{cases}
$$

On the other hand, $r_{3}(N)$ is the $N$-th Fourier coefficient of $\theta(z)^{3}$, where

$$
\theta(z)=\sum_{t \in \mathbb{Z}} q^{t^{2}} \quad\left(q=e^{2 \pi i z}\right)
$$

is a modular form of weight one-half; thus one should expect that the function

$$
\mathscr{H}(z)=\sum_{N=0}^{\infty} H(N) q^{N} \quad(z \in \mathfrak{H})
$$

is a modular form of weight $3 / 2$, and then the number $H_{1}(N)$ would be the $4 N$-th Fourier coefficient of the modular form $\mathscr{H}(z) \theta(z)$ of weight 2.

At the time of appearance of Hecke's paper, no satisfactory theory of modular forms of half-integral weight was known; such a theory has now been provided by Shimura $([33,34])$. However, one still cannot carry out Hecke's suggestion directly because, as we shall see, the function $\mathscr{H}(z)$ does not in fact transform like a modular form of weight $3 / 2$. For $r>1$ odd, on the other hand, Cohen proves that the function $\sum_{N=0}^{\infty} H(r, N) q^{N}$ is a modular form of weight $r+\frac{1}{2}$ (for $\left.\Gamma_{0}(4)\right)$ in the sense of Shimura, namely equal to the linear combination

$$
\frac{\zeta(1-2 r)}{2^{2 r+1}}\left\{(1-i) E_{r+1 / 2}(z)-i F_{r+1 / 2}(z)\right\}
$$

of the two Eisenstein series

$$
\begin{aligned}
& E_{r+1 / 2}(z)=\sum_{\substack{m=1 \\
m \text { odd }}}^{\infty} \sum_{\substack{n=-\infty \\
(n, m)=1}}^{\infty} \frac{\left(\frac{n}{m}\right)\left(\frac{-1}{m}\right)^{1 / 2}}{(m z+n)^{r+1 / 2}}, \\
& F_{r+1 / 2}(z)=z^{-r-1 / 2} E_{r+1 / 2}\left(\frac{-1}{4 z}\right),
\end{aligned}
$$

whose Fourier coefficients were calculated by Shimura in the papers cited.

For $r=1$ we should like to apply the same idea and show that $\mathscr{H}(z)$ is equal to the linear combination

$$
\mathscr{F}(z)=-\frac{1}{96}\left\{(1-i) E_{3 / 2}(z)-i F_{3 / 2}(z)\right\}
$$

of the two Eisenstein series of weight $3 / 2$. However, the series defining $E_{r+1 / 2}(z)$ diverges for $r=1$. To overcome this difficulty, we use the well-known device 
of Hecke [25]: we introduce the series

$$
E_{3 / 2, s}(z)=\sum_{\substack{m>0 \\(m, 2 n)=1}} \frac{\left(\frac{n}{m}\right)\left(\frac{-1}{m}\right)^{1 / 2}}{(m z+n)^{3 / 2}|m z+n|^{2 s}} \quad(z \in \mathfrak{H}, s \in \mathbb{C}),
$$

which converges absolutely for $\operatorname{Re}(s)>\frac{1}{4}$ and transforms by

$$
E_{3 / 2, s}\left(\frac{a z+b}{c z+d}\right)=\left(\frac{c}{d}\right)\left(\frac{-1}{d}\right)^{1 / 2}(c z+d)^{3 / 2}|c z+d|^{2 s} E_{3 / 2, s}(z)
$$

for $\left(\begin{array}{ll}a & b \\ c & d\end{array}\right) \in \Gamma_{0}(4)$ (for the definitions of $\left(\frac{c}{d}\right),\left(\frac{-1}{d}\right)^{1 / 2}$ etc. cf. [33]). This function is analytic in $S$, and by analytic continuation we obtain a function $E_{3 / 2}(z)=E_{3 / 2,0}(z)$ which is possibly not holomorphic in $z$ but a least satisfies the transformation equation of a modular form of weight $3 / 2$. We proceed similarly for $F_{3 / 2}(z)$ and then define $\mathscr{F}(z)$ by $(1)$. The function $\mathscr{F}(z)$ is periodic of period 1 and hence has a Fourier expansion $\sum f_{N} e^{2 \pi i N z}$, the coefficients $f_{N}$ possibly being functions of $y=\operatorname{Im}(z)$. We will calculate these Fourier coefficients in the next section, finding that the $N$-th coefficient is equal to $H(N)$ (independent of $y$ ) for $N$ positive and to 0 for $N$ negative except for $N=-u^{2}, u \in \mathbb{Z}$. Thus $\mathscr{F}(z)$ is the sum of $\mathscr{H}(z)$ and a certain non-analytic expression involving the powers $q^{-u^{2}}$. In Section 2.3 we construct a theta series of weight 2 which will cancel the contribution from this non-analytic piece and create the term $\sum \min \left(\lambda, \lambda^{\prime}\right)$ in the formula for $c(N)$. The proof of Theorem 1 will be completed in Section 2.4.

\subsection{The Eisenstein Series of Weight $\frac{3}{2}$}

At the end of the last section we defined a function $\mathscr{F}(z)$ which transforms under $\Gamma_{0}(4)$ like a modular form of weight $\frac{3}{2}$, and explained a reason for expecting a relationship between $\mathscr{F}(z)$ and the function $\mathscr{H}(z)=\sum H(N) q^{N}$. In this section we will prove the following result.

Theorem 2. For $z \in \mathfrak{H}$, we have

$$
\mathscr{F}(z)=\mathscr{H}(z)+y^{-1 / 2} \sum_{f=-\infty}^{\infty} \beta\left(4 \pi f^{2} y\right) q^{-f^{2}},
$$

where $y=\operatorname{Im}(z), q=\mathrm{e}(z)$ and $\beta(x)$ is defined by

$$
\beta(x)=\frac{1}{16 \pi} \int_{1}^{\infty} u^{-3 / 2} e^{-x u} d u \quad(x \geqq 0) .
$$

Before proving this, we mention two corollaries. The first is a description of the way $\mathscr{H}(z)$ transforms under $\Gamma_{0}(4)$. 
Corollary. For $\left(\begin{array}{ll}a & b \\ c & d\end{array}\right) \in \Gamma_{0}(4), c \neq 0$,

$$
\left(\frac{c}{d}\right)\left(\frac{-1}{d}\right)^{-1 / 2}(c z+d)^{3 / 2} \mathscr{H}\left(\frac{a z+b}{c z+d}\right)-\mathscr{H}(z)=\frac{1+i}{16 \pi} \int_{d / c}^{i \infty} \frac{\theta(t) d t}{(t+z)^{3 / 2}}
$$
where $\theta(t)=\sum_{f \in \mathbb{Z}} \mathrm{e}\left(f^{2} t\right)$ and the integral is taken along a vertical path in the upper
half-plane.

Indeed, by the theorem,

$$
\mathscr{F}(z)-\mathscr{H}(z)=\frac{1}{16 \pi} y^{-1 / 2} \int_{1}^{\infty} u^{-3 / 2} \theta(2 i u y-z) d u=\frac{1+i}{16 \pi} \int_{-\bar{z}}^{i \infty}(z+v)^{-3 / 2} \theta(v) d v,
$$

the integral being taken along the vertical path $v=2 i u y-z, 1 \leqq u<\infty$. Denote the latter integral by $\psi(z)$; then, substituting $v=\frac{a t-b}{-c t+d}$, we find

$$
\begin{aligned}
\psi\left(\frac{a z+b}{c z+d}\right) & =\int_{-\bar{z}}^{d / c}\left(\frac{a z+b}{c z+d}+\frac{a t-b}{-c t+d}\right)^{-3 / 2} \theta\left(\frac{a t-b}{-c t+d}\right) \frac{d t}{(c t-d)^{2}} \\
& =\left(\frac{-c}{d}\right)\left(\frac{-1}{d}\right)^{-1 / 2}(c z+d)^{3 / 2} \int_{-\bar{z}}^{d / c}(z+t)^{-3 / 2} \theta(t) d t,
\end{aligned}
$$

where in the second line we have used our knowledge of the behaviour of $\theta(t)$ under $\Gamma_{0}(4)$. Thus

$$
\left(\frac{c}{d}\right)\left(\frac{-1}{d}\right)^{-1 / 2}(c z+d)^{-3 / 2} \psi\left(\frac{a z+b}{c z+d}\right)-\psi(z)=-\int_{d / c}^{i \infty}(z+t)^{-3 / 2} \theta(t) d t .
$$

The expression on the left, with $\psi$ replaced by $\mathscr{F}$, is zero because $\mathscr{F}$ transforms under $\Gamma_{0}(4)$ like a modular form of weight $3 / 2$. The Corollary now follows from the identity $\mathscr{F}-\mathscr{H}=\frac{1+i}{16 \pi} \psi$.

We should mention that one result concerning the behaviour of $\mathscr{H}$ under modular transformations was already known, namely the identity

$$
(2 z / i)^{-3 / 2} \mathscr{H}\left(\frac{-1}{4 z}\right)+\mathscr{H}(z)=-\frac{1}{24} \theta(z)^{3}-\sqrt{\frac{z}{8 i}} \int_{-\infty}^{\infty} \mathrm{e}\left(\xi^{2} z\right) \frac{1+\mathrm{e}(2 \xi z)}{1-\mathrm{e}(2 \xi z)} \xi d \xi,
$$

found by Eichler [21].

The other consequence of Theorem 2 was pointed out to us by H. Cohen, namely, a "modular" proof of the Gauss-Hermite formula quoted in Section 2.1. To see that $r_{3}(8 N+3)=24 H(8 N+3)$, for example, we observe that

$$
\sum H(8 N+3) q^{N}=\frac{1}{8} \sum_{r(\bmod 8)} \mathrm{e}(-3 r / 8) \mathscr{H}\left(\frac{z+r}{8}\right)=\frac{1}{8} \sum_{r(\bmod 8)} \mathrm{e}(-3 r / 8) \mathscr{F}\left(\frac{z+r}{8}\right),
$$

the terms involving $q^{-f^{2}}$ all dropping out because $-f^{2}$ is never congruent to 3 modulo 8 . Therefore the function $\sum H(8 N+3) q^{N}$ is a (holomorphic) modular form of weight $3 / 2$ for some congruence group (in fact for $\Gamma_{0}(2)$ ), and since 
$\frac{1}{24} \sum r_{3}(8 N+3) q^{N}$ is also such a form, one can prove the equality of the two functions by comparing finitely many of their coefficients. A similar argument works for coefficients belonging to the sequence $4 N+2$ or $4 N+3$ or to any other arithmetical progression not containing the negatives of any squares.

We now give the proof of Theorem 2. Set

$$
\mathscr{F}_{s}(z)=-\frac{1}{96}\left\{(1-i) E_{3 / 2, s}(z)-i F_{3 / 2, s}(z)\right\} \quad\left(\operatorname{Re}(s)>\frac{1}{4}\right),
$$

where $E_{3 / 2, s}(z)$ is the function defined by Eq. (2) of 2.1 and

$$
F_{3 / 2, s}(z)=z^{-3 / 2}|z|^{-2 s} E_{3 / 2, s}(-1 / 4 z) \text {. }
$$

Then $\mathscr{F}_{s}(z)$ has an analytic continuation to the whole $s$-plane with $\mathscr{F}_{0}(z)=\mathscr{F}(z)$, and on the other hand $\mathscr{F}_{s}$ is periodic in $z$ with period 1 and therefore has a Fourier development of the form

$$
\mathscr{F}_{s}(z)=\sum_{N=-\infty}^{\infty} f_{N}(s, y) q^{N}
$$

with $f_{N}(s, y)$ analytic in $s$. Theorem 2 will follow if we show

$$
f_{N}(0, y)= \begin{cases}H(N) & \text { if } N>0, \\ -\frac{1}{12}+\frac{1}{8 \pi} y^{-1 / 2} & \text { if } N=0, \\ 2 y^{-1 / 2} \beta\left(4 \pi f^{2} y\right) & \text { if } N=-f^{2}, f>0, \\ 0 & \text { if } N<0,-N \neq \text { square }\end{cases}
$$

We begin by finding the Fourier expansion of $E_{3 / 2, s}(z)$. Write

$$
E_{3 / 2, s}(z)=\sum_{\substack{m=1 \\ m \text { odd }}}^{\infty}\left(\frac{-1}{m}\right)^{1 / 2} m^{-3 / 2-2 s} \sum_{n(\bmod m)}\left(\frac{n}{m}\right) \sum_{h \in \mathbb{Z}}\left(z+\frac{n}{m}+h\right)^{-3 / 2}\left|z+\frac{n}{m}+h\right|^{-2 s} .
$$

By the Poisson summation formula,

$$
\sum_{h \in \mathbb{Z}}(z+h)^{-3 / 2}|z+h|^{-2 s}=\sum_{N=-\infty}^{\infty} \alpha_{N}(s, y) \mathrm{e}(N z) \quad(z \in \mathfrak{H})
$$

with

$$
\begin{aligned}
\alpha_{N}(s, y) & =\int_{i y-\infty}^{i y+\infty} z^{-3 / 2}|z|^{-2 s} \mathrm{e}(-N z) d z \\
& =y^{-1 / 2-2 s} e^{2 \pi N y} \int_{-\infty}^{\infty}(v+i)^{-3 / 2}\left(v^{2}+1\right)^{-s} \mathrm{e}(-N y v) d v
\end{aligned}
$$

(the last formula is obtained by the substitution $z=(v+i) y$ ), and inserting this into the formula for $E_{3 / 2, s}$ we find

$$
\begin{aligned}
E_{3 / 2, s}(z) & =\sum_{\substack{m=1 \\
m \text { odd }}}^{\infty} m^{-1-2 s} \sum_{N=-\infty}^{\infty} \gamma_{m}(-N) \alpha_{N}(s, y) \mathrm{e}(N z) \\
& =\sum_{N=-\infty}^{\infty} E_{-N}^{\text {odd }}(1+2 s) \alpha_{N}(s, y) e^{2 \pi i N z},
\end{aligned}
$$


where $\gamma_{m}(-N)$ denotes the Gauss sum

$$
\gamma_{m}(-N)=\left(\frac{-1}{m}\right)^{1 / 2} m^{-1 / 2} \sum_{n(\bmod m)}\left(\frac{n}{m}\right) \mathrm{e}(n N / m) \quad(m \text { odd })
$$

and $E_{-N}^{\text {odd }}(s)$ the Dirichlet series

$$
E_{-N}^{\text {odd }}(s)=\sum_{\substack{m=1 \\ m \text { odd }}}^{\infty} \gamma_{m}(-N) m^{-s} .
$$

A similar calculation for $F_{3 / 2, s}(z)$ gives

with

$$
F_{3 / 2, s}(z)=2^{2 s+3} i+(1+i) \sum_{N=-\infty}^{\infty} E_{-N}^{\text {even }}(1+2 s) \alpha_{N}(s, y) e^{2 \pi i N z}
$$

$$
E_{-N}^{\text {even }}(s)=\sum_{\substack{m=1 \\ m \text { even }}}^{\infty} \gamma_{m}(-N)(m / 2)^{-s},
$$

the Gauss sum $\gamma_{m}(-N)$ now being defined by

$$
\gamma_{m}(-N)=m^{-1 / 2} \sum_{n(\bmod 2 m)}\left(\frac{m}{n}\right) i^{n / 2} \mathrm{e}(N n / 2 m) \quad(m \text { even }) .
$$

(The constant term $2^{2 s+3} i$ comes from the term $m=1, n=0$ in (2).) Thus the Fourier expansion of $\mathscr{F}_{s}(z)$ is

$$
\mathscr{F}_{s}(z)=-\frac{2^{2 s}}{12}-\frac{1-i}{48} \sum_{N=-\infty}^{\infty} E_{-N}(1+2 s) \alpha_{N}(s, y) q^{N}
$$

with

$$
E_{-N}(s)=\frac{1}{2}\left(E_{-N}^{\text {odd }}(s)+E_{-N}^{\text {even }}(s)\right) .
$$

The Gauss sums $\gamma_{m}(-N)$ and the Dirichlet series $E_{-N}^{\text {odd }}, E_{-N}^{\text {even }}$ and $E_{-N}$ are evaluated in [15], $\S 4$, Theorems 2 and 3 . It turns out that $E_{-N}(s)=0$ identically if $N$ is congruent to 1 or $2(\bmod 4)$, while if $N \neq 0$ is congruent to 0 or $3(\bmod 4)$, then

$$
E_{-N}(s)=\zeta(2 s)^{-1} L\left(s, \chi_{d}\right) \sum_{\substack{a, c>0 \\ a c \mid s}} \mu(a) \chi_{d}(a) c^{-2 s+1} a^{-s},
$$

where $d$ is the discriminant of $\mathbb{Q}(\sqrt{-N}), \chi_{d}$ the associated character, and $f$ the number defined by $-N=d f^{2}$. Finally, for $N=0$ we have

$$
E_{0}(s)=\zeta(2 s-1) / \zeta(2 s) .
$$

We are interested in finding the value of the Fourier coefficients

$$
f_{N}(s, y)=-\frac{1-i}{48} E_{-N}(1+2 s) \alpha_{N}(s, y)+ \begin{cases}-2^{2 s} / 12 & \text { if } N=0 \\ 0 & \text { if } N \neq 0\end{cases}
$$

at $s=0$. 
The integral defining $\alpha_{N}(s, y)$ can be evaluated easily at $s=0$ :

$$
\alpha_{N}(0, y)= \begin{cases}-4 \pi(1+i) N^{1 / 2} & \text { if } N>0 \\ 0 & \text { if } N \leqq 0\end{cases}
$$

If $N$ is positive, formula (3) shows that $E_{-N}(s)$ is holomorphic at $s=1$ with

$$
\begin{aligned}
E_{-N}(1) & =\frac{6}{\pi^{2}} L\left(1, \chi_{d}\right) \sum_{a c \mid f} \mu(a) \chi_{d}(a) / a c \\
& =\frac{6}{\pi} N^{-1 / 2} L\left(0, \chi_{d}\right) \sum_{k \mid f} k \prod_{p \mid k}\left(1-\chi_{d}(p) / p\right) \\
& =\frac{6}{\pi} N^{-1 / 2} H(N)
\end{aligned}
$$

(we have used the functional equation of $L\left(s, \chi_{d}\right)$ ), so $f_{N}(0, y)=H(N)$ as claimed. If $N$ is negative but $-N$ is not a square, then the number $d$ in (3) is the discriminant of a real quadratic field and so $L\left(s, \chi_{d}\right)$ and hence $E_{-N}(s)$ are holomorphic at $s=1$; this, together with $\alpha_{N}(0, y)=0$, implies that $f_{N}(0, y)=0$. It remains to treat the case $N=-f^{2}$.

First, if $N=-f^{2}, f>0$, then

$$
E_{-N}(s)=\zeta(2 s)^{-1} \zeta(s) \sum_{a c \mid f} \mu(a) c^{-2 s+1} a^{-s}
$$

has a pole of residue $\zeta(2)^{-1}$ at $s=1$, so

$$
f_{N}(0, y)=-\frac{1-i}{16 \pi^{2}} \alpha_{N}^{\prime}(0, y)
$$

where

$$
\begin{aligned}
\alpha_{N}^{\prime}(0, y) & =\left.\frac{\partial}{\partial s} \alpha_{N}(s, y)\right|_{s=0} \\
& =-y^{-1 / 2} e^{2 \pi N y} \int_{-\infty}^{\infty}(v+i)^{-3 / 2} \log \left(v^{2}+1\right) \mathrm{e}(-N y v) d v \\
& =-(2 i y)^{-1 / 2} \int_{1 / 2-i \infty}^{1 / 2+i \infty} u^{-3 / 2} e^{4 \pi N y u} \log \{4 u(1-u)\} d u
\end{aligned}
$$

(the last equation is obtained by substituting $v=2 i u-i$ ). We deform the path of integration in the last integral to a path in the cut plane $\mathbb{C}-[1, \infty)$ which circles the cut clockwise from $i \varepsilon+\infty$ to $\frac{1}{2}$ to $i \varepsilon-\infty$. Across the cut, $\log \{4 u(1-u)\}$ jumps by $2 \pi i$ and the other terms in the integrand are continuous. Therefore

$$
\alpha_{N}^{\prime}(0, y)=-2 \pi i(2 i y)^{-1 / 2} \int_{1}^{\infty} u^{-3 / 2} e^{-4 \pi|N| y u} d u=-16 \pi^{2}(1+i) y^{-1 / 2} \beta(4 \pi|N| y)
$$

and hence

$$
f_{N}(0, y)=2 y^{-1 / 2} \beta\left(4 \pi f^{2} y\right) \quad\left(N=-f^{2}<0\right)
$$


as claimed. Finally, for $N=0$ we have

$$
f_{0}(0, y)=-\frac{1}{12}-\frac{1-i}{32 \pi^{2}} \alpha_{0}^{\prime}(0, y)=-\frac{1}{12}-\frac{1}{8 \pi} y^{-1 / 2},
$$

because $E_{0}(s)=\zeta(2 s-1) / \zeta(2 s)$ has a pole of residue $3 / \pi^{2}$ at $s=1$. This completes the proof of Theorem 2 .

\subsection{A Theta-Series Attached to an Indefinite Quadratic Form}

If $F$ is a positive definite quadratic form in $2 k$ variables, and $L \subset \mathbb{R}^{2 k}$ some lattice on which $F$ takes integral values, then the associated theta-series $\sum_{\lambda \in L} e^{2 \pi i z F(\lambda)}(z \in \mathfrak{G})$ is a modular form of weight $k$ (of some level and for some quadratic character depending on $F$ and $L$ ); similarly, the series $\sum p(\lambda) e^{2 \pi i z F(\lambda)}$, where $p(\lambda)$ is a homogeneous polynomial of degree $m$ which is spherical with respect to $F$, is a modular form of weight $k+m$ (for precise statements and proofs see [30] or [24]). If, on the other hand, $F$ is an indefinite form, then these series diverge because $\left|e^{2 \pi i z F(\lambda)}\right|$ grows exponentially in $\left|\lambda^{2}\right|$ in the cone $F(\lambda)<0$. To obtain a convergent series, we can either allow the coefficient $p(\lambda)$ to be a non-analytic (or piecewise analytic) homogeneous function of $\lambda$ which is identically zero for $F(\lambda)<0$, or allow $p(\lambda)=p_{z}(\lambda)$ to depend on $z$ in such a way that $p_{z}(\lambda)$ is much smaller than $e^{-2 \pi i z F(\lambda)}$ as $|\lambda| \rightarrow \infty$. If the function $p_{z}(\lambda)$ is chosen in such a way that the Fourier transform (with respect to $\lambda)$ of $p_{z}(\lambda) e^{2 \pi i z F(\lambda)}$ equals $z^{-r} p_{-1 / z}(\lambda) e^{-2 \pi i F(\lambda) / z}$ for some $r$, then the same proof as in the classical case (namely, by application of the Poisson summation formula) shows that $\sum p_{z}(\lambda) e^{2 \pi i z F(\lambda)}$ is a modular form of weight $r$. In this section we shall construct such a modular form, of weight 2 , associated to the norm form of the quadratic field $K$, i.e. $F$ will be the indefinite form $F\left(\lambda_{1}, \lambda_{2}\right)=\lambda_{1} \lambda_{2}$ on $\mathbb{R}^{2}$ and $L$ will be the lattice $\mathcal{O}$, embedded in $\mathbb{R}^{2}$ by $x \mapsto\left(x, x^{\prime}\right)$. Our coefficient function $p_{z}\left(\lambda_{1}, \lambda_{2}\right)$ will be

$$
\frac{2}{\sqrt{y}} \beta\left(\pi y\left(\lambda_{1}-\lambda_{2}\right)^{2}\right)- \begin{cases}\frac{1}{2} \min \left(\left|\lambda_{1}\right|,\left|\lambda_{2}\right|\right) & \text { if } \lambda_{1} \lambda_{2}>0, \\ 0 & \text { if } \lambda_{1} \lambda_{2} \leqq 0,\end{cases}
$$

where

$$
\beta(x)=\frac{1}{16 \pi} \int_{1}^{\infty} u^{-3 / 2} e^{-x u} d u \quad(\operatorname{Re} x \geqq 0)
$$

is the function defined in Theorem 2.

We will need some properties of the function $\beta(x)$ and of the related function

$$
f(a, x)=\int_{x}^{\infty} e^{-u^{2}+2 \pi i a u} \frac{d u}{u} \quad\left(a \in \mathbb{C}, x \in \mathbb{C}-\mathbb{R}_{-}\right) .
$$

These functions are related to the standard "complementary error function"

$$
\operatorname{erfc}(x)=\frac{2}{\sqrt{\pi}} \int_{x}^{\infty} e^{-u^{2}} d u \quad(x \in \mathbb{C})
$$


by the formulas

$$
\beta(x)=\frac{1}{8 \pi}\left(e^{-x}-\sqrt{\pi x} \operatorname{erfc}(\sqrt{x})\right)
$$

(which also gives the analytic continuation of $\beta(x)$ to $\mathbb{C}-\mathbb{R}_{-}$) and

$$
\frac{\partial f(a, x)}{\partial a}=i \pi^{3 / 2} e^{-\pi^{2} a^{2}} \operatorname{erfc}(x+i \pi a) .
$$

The properties we need are summarized in the following lemma.

Lemma 1. The functions $\beta, f$ and erfc satisfy the identities
a) $\int_{-\infty}^{\infty} \beta\left(c t^{2}\right) e^{2 \pi i t x} d t=\frac{1}{16} \pi^{-5 / 2} c^{1 / 2} \frac{1-e^{-\pi^{2} x^{2} / c}}{x^{2}}$,
b) $\int_{-\infty}^{\infty} \frac{1-e^{-\alpha x^{2}}}{x^{2}} e^{2 \pi i t x} d x=16 \pi^{3 / 2} \alpha^{1 / 2} \beta\left(\pi^{2} t^{2} / \alpha\right)$,
c) $\operatorname{erfc}(x)+\operatorname{erfc}(-x)=2$,
d) $f(-a,-x)-f(a, x)=i \pi \operatorname{erfc}(\pi a)(x \in \mathfrak{H})$.

Proof. a) is obtained easily by substituting the definition of $\beta$ into the integral and inverting the order of integration, and b) by differentiating both sides of the identity with respect to $\alpha$; alternatively, b) can be considered as the inverse Fourier transform formula to a). Formula c) is standard and easy: one sees by differentiating the left-hand side that it is a constant, and the constant is found by setting $x=0$. Finally, the function $f(-a,-x)-f(a, x)$ is defined for $a \in \mathbb{C}$, $x \in \mathbb{C}-\mathbb{R}=\mathfrak{H} \cup-\mathfrak{H}$, and its derivative with respect to $x$ is identically zero, so for fixed $a$ this function has a constant value $\varphi_{+}(a)$ for $x$ in the upper half-plane and a constant value $\varphi_{-}(a)$ for $x$ in the lower half-plane. Differentiating with respect to $a$, we find

$$
\frac{d}{d a} \varphi_{ \pm}(a)=-2 i \pi^{3 / 2} e^{-\pi^{2} a^{2}}
$$

so

$$
\varphi_{ \pm}(a)=i \pi \operatorname{erfc}(\pi a)+c_{ \pm}
$$

for some complex constants $c_{+}$and $c_{-}$. Interchanging the roles of $(a, x)$ and $(-a,-x)$ in the definition of $\varphi_{ \pm}(a)$ leads to

$$
\varphi_{ \pm}(-a)=-\varphi_{\mp}(a) \text {, }
$$

which, together with c) of the lemma, implies $c_{+}+c_{-}=-2 \pi i$. Finally, it follows by the calculus of residues that

$$
\lim _{\varepsilon \searrow 0}(f(a, x-i \varepsilon)-f(a, x+i \varepsilon))=2 \pi i
$$

for $x$ real and negative, and this implies $c_{+}-c_{-}=2 \pi i$. Hence $c_{+}=0$ and $c_{-}=-2 \pi i$, so $\varphi_{ \pm}(a)= \pm i \pi \operatorname{erfc}( \pm \pi a)$.

We are now in a position to construct our non-analytic theta-series. 
Proposition 1. For $z \in \mathfrak{H}$, define continuous complex-valued functions $U_{z}, V_{z}$ and $W_{z}$ on $\mathbb{R}^{2}$ by

$$
\begin{aligned}
& U_{z}\left(\lambda, \lambda^{\prime}\right)=2 y^{-1 / 2} \beta\left(\pi y\left(\lambda-\lambda^{\prime}\right)^{2}\right) \mathrm{e}\left(\lambda \lambda^{\prime} z\right), \\
& V_{z}\left(\lambda, \lambda^{\prime}\right)= \begin{cases}\frac{1}{2} \min \left(|\lambda|,\left|\lambda^{\prime}\right|\right) \mathrm{e}\left(\lambda \lambda^{\prime} z\right) & \text { if } \lambda \lambda^{\prime}>0, \\
0 & \text { if } \lambda \lambda^{\prime} \leqq 0,\end{cases} \\
& W_{z}\left(\lambda, \lambda^{\prime}\right)=U_{z}\left(\lambda, \lambda^{\prime}\right)-V_{z}\left(\lambda, \lambda^{\prime}\right),
\end{aligned}
$$

where $y=\operatorname{Im}(z)$. Let $\tilde{W}$ denote the Fourier transform of $W$ :

$$
\tilde{W}_{z}\left(\mu, \mu^{\prime}\right)=\int_{-\infty}^{\infty} \int_{-\infty}^{\infty} W_{z}\left(\lambda, \lambda^{\prime}\right) \mathrm{e}\left(-\lambda \mu-\lambda^{\prime} \mu^{\prime}\right) d \lambda d \lambda^{\prime}
$$

Then

$$
\tilde{W}_{z}\left(\mu, \mu^{\prime}\right)=z^{-2} W_{-1 / z}\left(\mu, \mu^{\prime}\right) .
$$

Corollary. The function

$$
\mathscr{W}(z)=\sum_{\lambda \in \mathscr{O}} W_{z}\left(\lambda, \lambda^{\prime}\right) \quad(z \in \mathfrak{S})
$$

is a non-analytic modular form of weight 2 , level $D$ and Nebentypus $\chi_{D}$.

Proof. We calculate the Fourier transforms of $U_{z}$ and $V_{z}$ separately. First, in the integral defining $\tilde{U}_{z}$ we substitute $\lambda^{\prime}=\lambda+t$ and use a) of Lemma 1 :

$$
\begin{aligned}
\tilde{U}_{z}\left(\mu, \mu^{\prime}\right) & =\int_{-\infty}^{\infty} \int_{-\infty}^{\infty} \mathrm{e}\left(-\lambda \mu-\lambda^{\prime} \mu^{\prime}\right) U_{z}\left(\lambda, \lambda^{\prime}\right) d \lambda^{\prime} d \lambda \\
& =2 y^{-1 / 2} \int_{-\infty}^{\infty} \mathrm{e}\left(\lambda\left(z \lambda-\mu-\mu^{\prime}\right)\right) \int_{-\infty}^{\infty} \beta\left(\pi y t^{2}\right) \mathrm{e}\left(\left(z \lambda-\mu^{\prime}\right) t\right) d t d \lambda \\
& =\frac{1}{8 \pi^{2}} \int_{-\infty}^{\infty} \frac{1-e^{-\pi\left(z \lambda-\mu^{\prime}\right)^{2} / y}}{\left(z \lambda-\mu^{\prime}\right)^{2}} \mathrm{e}\left(z \lambda^{2}-\mu \lambda-\mu^{\prime} \lambda\right) d \lambda
\end{aligned}
$$

Substituting $\lambda=u+\mu^{\prime} / z$ and applying b) of Lemma 1 , we find

$$
\begin{aligned}
\tilde{U}_{z}\left(\mu, \mu^{\prime}\right) & =\frac{1}{8 \pi^{2} z^{2}} \mathrm{e}\left(-\mu \mu^{\prime} / z\right) \int_{-\infty}^{\infty} \frac{e^{2 \pi i z u^{2}}-e^{-\pi|z|^{2} u^{2} / y}}{u^{2}} \mathrm{e}\left(\left(\mu^{\prime}-\mu\right) u\right) d u \\
& =2 z^{-2} \mathrm{e}\left(-\mu \mu^{\prime} / z\right)\left\{|z| y^{-1 / 2} \beta\left(\pi y\left(\mu-\mu^{\prime}\right)^{2} /|z|^{2}\right)-w^{1 / 2} \beta\left(\pi\left(\mu-\mu^{\prime}\right)^{2} / w\right)\right\} \\
& =z^{-2} U_{-1 / z}\left(\mu, \mu^{\prime}\right)+8 w^{-3 / 2} \mathrm{e}\left(-\mu \mu^{\prime} / z\right) \beta\left(\pi\left(\mu-\mu^{\prime}\right)^{2} / w\right)
\end{aligned}
$$

where in the last two lines we have set $w=2 z / i$, so $|\arg (w)|<\frac{\pi}{2}$. For $\tilde{V}_{z}$ we find

$$
\tilde{V}_{z}\left(\mu, \mu^{\prime}\right)=T\left(\mu, \mu^{\prime}\right)+T\left(-\mu,-\mu^{\prime}\right)+T\left(\mu^{\prime}, \mu\right)+T\left(-\mu^{\prime},-\mu\right),
$$

where

$$
T\left(\mu, \mu^{\prime}\right)=\frac{1}{2} \iint_{0 \leqq \lambda \leqq \lambda^{\prime}} \lambda \mathrm{e}\left(z \lambda \lambda^{\prime}-\lambda \mu-\lambda^{\prime} \mu^{\prime}\right) d \lambda^{\prime} d \lambda
$$


In this expression we perform the $\lambda^{\prime}$-integration and substitute $\lambda=u+\mu^{\prime} / z$ to obtain

$$
\begin{aligned}
T\left(\mu, \mu^{\prime}\right)= & \frac{i}{4 \pi} \int_{0}^{\infty} \frac{\lambda}{z \lambda-\mu^{\prime}} \mathrm{e}\left(z \lambda^{2}-\mu \lambda-\mu^{\prime} \lambda\right) d \lambda \\
= & \frac{i}{4 \pi z} \mathrm{e}\left(-\mu \mu^{\prime} / z\right) \int_{-\mu^{\prime} / z}^{\infty} \frac{u+\mu^{\prime} / z}{u} \mathrm{e}\left(z u^{2}-u \mu+u \mu^{\prime}\right) d u \\
= & \frac{1}{4 \pi} w^{-3 / 2} \mathrm{e}\left(-\left(\mu-\mu^{\prime}\right)^{2} / 4 z\right) \operatorname{erfc}\left(i\left(\mu^{\prime}+\mu\right) \sqrt{\frac{\pi}{w}}\right) \\
& +\frac{i \mu^{\prime}}{4 \pi} z^{-2} \mathrm{e}\left(-\mu \mu^{\prime} / z\right) f\left(\frac{\mu^{\prime}-\mu}{\sqrt{\pi w}}, 2 i \mu^{\prime} \sqrt{\frac{\pi}{w}}\right) .
\end{aligned}
$$

Adding to this the corresponding formula for $T\left(-\mu,-\mu^{\prime}\right)$ and using c) and d) of Lemma 1, we find

$$
\begin{aligned}
& T\left(\mu, \mu^{\prime}\right)+T\left(-\mu,-\mu^{\prime}\right) \\
& =\frac{1}{2 \pi} w^{-3 / 2} \mathrm{e}\left(-\left(\mu-\mu^{\prime}\right)^{2} / 4 z\right)+\frac{1}{4}\left|\mu^{\prime}\right| z^{-2} \mathrm{e}\left(-\mu \mu^{\prime} / z\right) \operatorname{erfc}\left(\operatorname{sign}\left(\mu^{\prime}\right)\left(\mu^{\prime}-\mu\right) \sqrt{\frac{\pi}{w}}\right) .
\end{aligned}
$$

Adding to this the formula obtained by interchanging $\mu$ and $\mu^{\prime}$, and using again c) of the lemma as well as Equation (4), we find after a short calculation

$$
\tilde{V}_{z}\left(\mu, \mu^{\prime}\right)=z^{-2} V_{-1 / z}\left(\mu, \mu^{\prime}\right)+8 w^{-3 / 2} \mathrm{e}\left(-\mu \mu^{\prime} / z\right) \beta\left(\pi\left(\mu-\mu^{\prime}\right)^{2} / w\right) .
$$

Comparing this with the result for $\tilde{U}_{z}$ we obtain Equation (5).

The proof of the corollary is now essentially the same as the standard proof that theta-series associated to definite quadratic forms are modular forms, as given in [30], Chapter VI or [24], pages 81-87. We recall briefly how the argument goes. As well as the series $\mathscr{W}(z)$, one must consider the sums

$$
\mathscr{W}_{v}(z)=\sum_{\lambda \in \mathcal{O}} W_{z}\left(\lambda+v, \lambda^{\prime}+v^{\prime}\right)
$$

over the translated lattices $\mathcal{O}+v$, where $v$ belongs to the inverse different $\mathfrak{D}^{-1}=(1 / \sqrt{D})$. Clearly $\mathscr{W}_{v}$ depends only on the residue class of $v(\bmod \mathcal{O})$, so there are only $D$ distinct functions $\mathscr{W}_{v}$, with $\mathscr{W}_{0}=\mathscr{W}$. Then

$$
\mathscr{W}_{v}(z+1)=\sum_{\lambda \in \mathscr{O}} W_{z}\left(\lambda+v, \lambda^{\prime}+v^{\prime}\right) \mathrm{e}(\mathrm{N}(\lambda+v))=\mathrm{e}(\mathrm{N} v) \mathscr{W}_{v}(z)
$$

since $\mathrm{N}(\lambda+v)-\mathrm{N}(v) \in \mathbb{Z}$ for $\lambda \in \mathcal{O}, v \in \mathcal{D}^{-1}$. On the other hand, by the Poisson summation formula

$$
\mathscr{W}_{v}(z)=D^{-1 / 2} \sum_{\mu \in \mathrm{D}^{-1}} \tilde{W}_{z}\left(\mu, \mu^{\prime}\right) \mathrm{e}(\operatorname{Tr} \mu v),
$$

and combining this with (5) we find

Thus we have

$$
z^{-2} \mathscr{W}_{v}(-1 / z)=D^{-1 / 2} \sum_{\mu \in \mathbb{D}^{-1}} \mathrm{e}(\operatorname{Tr} \mu v) W_{z}\left(\mu, \mu^{\prime}\right)=D^{-1 / 2} \sum_{\mu \in \mathbb{D}^{-1 / \mathcal{O}}} \mathrm{e}(\operatorname{Tr} \mu v) \mathscr{W}_{\mu}(z)
$$

$$
\mathscr{W}_{v}\left|T=\mathrm{e}(\mathrm{N} v) \mathscr{W}_{\nu}, \quad \mathscr{W}_{v}\right| J=D^{-1 / 2} \sum_{\mu} \mathrm{e}(\operatorname{Tr} \mu \nu) \mathscr{W}_{\mu},
$$


where $T$ and $J$ are the matrices $\left(\begin{array}{ll}1 & 1 \\ 0 & 1\end{array}\right)$ and $\left(\begin{array}{rr}0 & 1 \\ -1 & 0\end{array}\right)$ in $\Gamma=S L_{2}(\mathbb{Z}) /\{ \pm 1\}$ and $\mathscr{W}_{v} \mid\left(\begin{array}{ll}a & b \\ c & d\end{array}\right)$ denotes the function $(c z+d)^{-2} \mathscr{W}_{v}\left(\frac{a z+b}{c z+d}\right)$. Since $T$ and $J$ generate $\Gamma$, we obtain a representation of $\Gamma$ in the space generated by the $D$ functions $\mathscr{W}_{v}$. The first step in the proof of the Corollary is to show that the Equations (6) imply $\mathscr{W}_{v} \mid A=\mathscr{W}_{v}$ für $A$ in the principal congruence group $\Gamma(D)$. The argument is given in [24], pages 85-87. Now to show that $\mathscr{W}_{0}$ transforms under $\Gamma_{0}(D)$ like a modular form of Nebentypus, we take $A=\left(\begin{array}{ll}a & b \\ c & d\end{array}\right) \in \Gamma_{0}(D)$ and set $R=T^{a} J T^{d} J T^{a} J$. Then $R \equiv\left(\begin{array}{ll}a & 0 \\ 0 & d\end{array}\right)(\bmod D)$, so, choosing $x \in \mathbb{Z}$ with $d x \equiv b(\bmod D)$, we find $A=A^{\prime} T^{x} R$ with $A^{\prime} \in \Gamma(D)$ and hence $\mathscr{W}_{0}\left|A=\mathscr{W}_{0}\right| R$. But from (6) we find

$$
\mathscr{W}_{v} \mid T^{a} J=D^{-1 / 2} \mathrm{e}(a \mathrm{~N}(v)) \sum_{\mu} \mathscr{W}_{\mu} \mathrm{e}(\operatorname{Tr} \mu v)
$$

and hence

$$
\begin{aligned}
\mathscr{W}_{v} \mid R & =\left(\left(\mathscr{W}_{v} \mid T^{a} J\right) \mid T^{d} J\right) \mid T^{a} J \\
& =D^{-3 / 2} \sum_{\mu} \sum_{\lambda} \sum_{\kappa} \mathrm{e}(a \mathrm{~N} v+d \mathrm{~N} \mu+a \mathrm{~N} \lambda+\operatorname{Tr}(\mu \nu+\lambda \mu+\kappa \lambda)) \mathscr{W}_{\kappa} \\
& =D^{-3 / 2} \sum_{\kappa} \mathscr{W}_{\kappa} \sum_{\lambda} \mathrm{e}\left(\operatorname{Tr} \lambda^{\prime}\left(\kappa-a v^{\prime}\right)\right) \sum_{\mu} \mathrm{e}\left(d \mathrm{~N}\left(\mu+a \lambda^{\prime}+a v^{\prime}\right)\right) .
\end{aligned}
$$

Replacing $\mu+a \lambda^{\prime}+a v^{\prime}$ by $\mu$ in the inner sum, we see that this sum is equal to the standard Gauss sum

$$
\sum_{\mu \in D-1 / 0} \mathrm{e}(d \mathrm{~N} \mu)=D^{1 / 2} \chi_{D}(d) .
$$

Hence

$$
\mathscr{W}_{v} \mid R=D^{-1} \chi_{D}(d) \sum_{\kappa} \mathscr{W}_{\kappa} \sum_{\lambda} \mathrm{e}\left(\operatorname{Tr} \lambda^{\prime}\left(\kappa-a v^{\prime}\right)\right)=\chi_{D}(d) \mathscr{W}_{a v^{\prime}}
$$

(the inner sum is zero if $\kappa \neq a v^{\prime}$ ). In particular, taking $v=0$ we find $\mathscr{W}_{0} \mid R=\chi_{D}(d) \mathscr{W}_{0}$, and this completes the proof that $\mathscr{W}_{0} \mid A=\chi_{D}(d) \mathscr{W}_{0}$ for $A=\left(\begin{array}{ll}a & b \\ c & d\end{array}\right) \in \Gamma_{0}(D)$.

\subsection{Proof of Theorem 1}

We proved in Section 2.2 the identity

$$
\mathscr{F}(z)=\sum_{N=0}^{\infty} H(N) q^{N}+y^{-1 / 2} \sum_{u \in \mathbb{Z}} \beta\left(4 \pi u^{2} y\right) q^{-u^{2}},
$$

where as usual $q=\mathrm{e}(z)$ and $y=\operatorname{Im}(z)$ and $\mathscr{F}$ is a function satisfying

$$
\mathscr{F}\left(\frac{a z+b}{c z+d}\right)=\left(\frac{-1}{d}\right)^{1 / 2}\left(\frac{c}{d}\right)(c z+d)^{3 / 2} \mathscr{F}(z)
$$


for $\left(\begin{array}{ll}a & b \\ c & d\end{array}\right) \in \Gamma_{0}(4)$ (and of course $\left.c \neq 0\right)$. The function $\theta(z)=\sum_{t \in \mathbb{Z}} q^{t^{2}}$, on the other hand, satisfies

$$
\theta\left(\frac{a z+b}{c z+d}\right)=\left(\frac{-1}{d}\right)^{-1 / 2}\left(\frac{c}{d}\right)(c z+d)^{1 / 2} \theta(z)
$$

for $\left(\begin{array}{ll}a & b \\ c & d\end{array}\right) \in \Gamma_{0}(4)$. It follows immediately that

$$
\begin{aligned}
\mathscr{F}( & \left.D \frac{a z+b}{c z+d}\right) \theta\left(\frac{a z+b}{c z+d}\right) \\
& =\mathscr{F}\left(\frac{a D z+b D}{(c / D) D z+d}\right) \theta\left(\frac{a z+b}{c z+d}\right) \\
& =\left(\frac{-1}{d}\right)^{1 / 2}\left(\frac{c / D}{d}\right)(c z+d)^{3 / 2} \mathscr{F}(D z)\left(\frac{-1}{d}\right)^{-1 / 2}\left(\frac{c}{d}\right)(c z+d)^{1 / 2} \theta(z) \\
& =\left(\frac{D}{d}\right)(c z+d)^{2} \mathscr{F}(D z) \theta(z)
\end{aligned}
$$

for $\left(\begin{array}{ll}a & b \\ c & d\end{array}\right) \in \Gamma_{0}(4 D)$, i.e. the function

$$
\begin{aligned}
& \mathscr{F}(D z) \theta(z)=\sum_{N=-\infty}^{\infty} c_{N}(y) q^{N}, \\
& c_{N}(y)=\sum_{\substack{t^{2} \leq N \\
t^{2} \equiv N(\bmod D)}} H\left(\frac{N-t^{2}}{D}\right)+D^{-1 / 2} y^{-1 / 2} \sum_{\substack{t, u \in \mathbb{Z} \\
t^{2}-D u^{2}=N}} \beta\left(4 \pi u^{2} y\right),
\end{aligned}
$$

belongs to $M_{2}^{*}\left(\Gamma_{0}(4 D), \chi_{D}\right)$. We claim that the function $\sum c_{4 N}\left(\frac{1}{4} y\right) q^{N}$ belongs to $M_{2}^{*}\left(\Gamma_{0}(D), \chi_{D}\right)$. The corresponding statement in [23] (Proposition 5.1) is proved by appealing to Lemmas 1 and 4 of [29]; however, since this latter paper treats only analytic modular forms, we give the proof of the special assertion we need:

Lemma 2. If $f(z)=\sum_{n \in \mathbb{Z}} a_{n}(y) q^{n}$ is in $M_{k}^{*}\left(\Gamma_{0}(4 D), \chi_{D}\right)$, and $a_{n}(y)=0$ for all $n \equiv 2(\bmod 4)$,
then the function

$$
h(z)=\frac{1}{4} \sum_{r=1}^{4} f\left(\frac{z+r}{4}\right)=\sum_{n \in \mathbb{Z}} a_{4 n}\left(\frac{1}{4} y\right) q^{n}
$$

is in $M_{k}^{*}\left(\Gamma_{0}(D), \chi_{D}\right)$.

Proof. We prove the lemma in two steps, first showing that the function

$$
g(z)=\frac{1}{2}\left(f\left(\frac{z}{2}\right)+f\left(\frac{z+1}{2}\right)\right)=\sum a_{2 n}\left(\frac{1}{2} y\right) q^{n}
$$


is in $M_{k}^{*}\left(\Gamma_{0}(2 D), \chi_{D}\right)$. Set $X_{0}=\left(\begin{array}{ll}1 & 0 \\ 0 & 2\end{array}\right), X_{1}=\left(\begin{array}{ll}1 & 1 \\ 0 & 2\end{array}\right)$ and let $A=\left(\begin{array}{ll}a & b \\ c & d\end{array}\right)$ be any matrix in $\Gamma_{0}(2 D)$. If $b$ is even, then the matrices

$$
X_{0} A X_{0}^{-1}=\left(\begin{array}{cc}
a & \frac{1}{2} b \\
2 c & d
\end{array}\right), \quad X_{1} A X_{1}^{-1}=\left(\begin{array}{cc}
a+c & \frac{1}{2}(b+d-a-c) \\
2 c & d-c
\end{array}\right)
$$

are in $\Gamma_{0}(4 D)$, so

$$
\begin{aligned}
g(A z) & =\frac{1}{2} f\left(X_{0} A X_{0}^{-1}\left(X_{0} z\right)\right)+\frac{1}{2} f\left(X_{1} A X_{1}^{-1}\left(X_{1} z\right)\right) \\
& =\frac{1}{2} \chi_{D}(d)(c z+d)^{k} f\left(X_{0} z\right)+\frac{1}{2} \chi_{D}(d-c)(c z+d)^{k} f\left(X_{1} z\right) \\
& =\chi_{D}(d)(c z+d)^{k} g(z),
\end{aligned}
$$

the last equality holding because $\chi_{D}(d-c)=\chi_{D}(d)$. If $b$ is odd, then the matrices

$$
X_{0} A X_{1}^{-1}=\left(\begin{array}{cc}
a & \frac{1}{2}(b-a) \\
2 c & d-c
\end{array}\right), \quad X_{1} A X_{0}^{-1}=\left(\begin{array}{cc}
a+c & \frac{1}{2}(b+d) \\
2 c & d
\end{array}\right)
$$

are in $\Gamma_{0}(4 D)$, so

$$
\begin{aligned}
g(A z) & =\frac{1}{2} f\left(X_{0} A X_{1}^{-1}\left(X_{1} z\right)\right)+\frac{1}{2} f\left(X_{1} A X_{0}^{-1}\left(X_{0} z\right)\right) \\
& =\frac{1}{2} \chi_{D}(d-c)(c z+d)^{k} f\left(X_{1} z\right)+\frac{1}{2} \chi_{D}(d)(c z+d)^{k} f\left(X_{0} z\right) \\
& =\chi_{D}(d)(c z+d)^{k} g(z) .
\end{aligned}
$$

This proves the assertion concerning $g$. Under the assumptions made on $f$, $a_{2 n}\left(\frac{1}{2} y\right)=0$ for $n$ odd, so

$$
g(z)=g\left(z+\frac{1}{2}\right)=\sum a_{4 n}\left(\frac{1}{2} y\right) q^{2 n}=h(2 z) .
$$

Let $A=\left(\begin{array}{ll}a & b \\ c & d\end{array}\right) \in \Gamma_{0}(D)$. The formulas just given for the four matrices $X_{\alpha} A X_{\beta}^{-1}$ $(\alpha, \beta=0,1)$ show that at least one of these matrices lies in $\Gamma_{0}(2 D)$; then

$$
h(A z)=g\left(X_{\alpha} A X_{\beta}^{-1}\left(X_{\beta} z\right)\right)=\chi_{D}(d)(c z+d)^{k} g\left(X_{\beta} z\right)=\chi_{D}(d)(c z+d)^{k} h(z) .
$$

We now apply this lemma with $f(z)=\mathscr{F}(z) \theta(z), a_{n}(y)=c_{n}(y)$. The fact that $c_{N}(y)=0$ for $N \equiv 2(\bmod 4)$ follows from the fact $H(n)=0$ unless $n \equiv 0$ or $3(\bmod 4)$ and from $D \equiv 0$ or $1(\bmod 4)$. Thus $\sum c_{4 N}\left(\frac{1}{4} y\right) q^{N}$ is in $M_{2}^{*}\left(\Gamma_{0}(D), \chi_{D}\right)$. But

$$
\begin{aligned}
c_{4 N}\left(\frac{1}{4} y\right)= & \sum_{\substack{t^{2} \leq 4 N \\
t^{2} \equiv 4 \bar{N}(\bmod D)}} H\left(\frac{4 N-t^{2}}{D}\right)+2 D^{-1 / 2} y^{-1 / 2} \sum_{\substack{t, u \in \mathbf{Z} \\
N=\left(t^{2}-D u^{2}\right) / 4}} \beta\left(\pi D u^{2} y\right) \\
= & H_{D}(N)+2 D^{-1 / 2} y^{-1 / 2} \sum_{\substack{\lambda \in \mathcal{C} \\
\lambda \lambda^{\prime}=N}} \beta\left(\pi\left(\lambda-\lambda^{\prime}\right)^{2} y\right),
\end{aligned}
$$

so this means that the function

$$
\sum_{N=0}^{\infty} H_{D}(N) q^{N}+2 D^{-1 / 2} y^{-1 / 2} \sum_{\lambda \in \mathcal{O}} \beta\left(\pi\left(\lambda-\lambda^{\prime}\right)^{2} y\right) q^{\lambda \lambda^{\prime}}
$$


is in $M_{2}^{*}\left(\Gamma_{0}(D), \chi_{D}\right)$. On the other hand, by the Corollary to Proposition 1, we know that the function

$$
-D^{-1 / 2} \mathscr{W}(z)=D^{-1 / 2} \sum_{\substack{\lambda \in \mathcal{O} \\ \lambda \gg 0}} \min \left(\lambda, \lambda^{\prime}\right) q^{\lambda \lambda^{\prime}}-2 D^{-1 / 2} y^{-1 / 2} \sum_{\lambda \in \mathcal{O}} \beta\left(\pi\left(\lambda-\lambda^{\prime}\right)^{2} y\right) q^{\lambda \lambda^{\prime}}
$$

is also in $M_{2}^{*}\left(\Gamma_{0}(D), \chi_{D}\right)$. Adding these two functions, we find that the function

$$
\varphi_{D}(z)=\sum_{N=0}^{\infty} H_{D}(N) q^{N}+D^{-1 / 2} \sum_{\substack{\lambda \in \mathcal{O} \\ \lambda \gg 0}} \min \left(\lambda, \lambda^{\prime}\right) q^{\lambda \lambda^{\prime}}
$$

is in $M_{2}^{*}\left(\Gamma_{0}(D), \chi_{D}\right)$. Since the $N$-th Fourier coefficient of $\varphi_{D}(z)$ is independent of $y$ and is $O\left(N^{r}\right)$ for some $r$ (in fact, for $r=1$ ), the function $\varphi_{D}(z)$ is analytic in the upper half-plane and is $O\left(y^{-r}\right)$ as $y \rightarrow 0$, which implies that $\varphi_{D}(z)$ is holomorphic at the cusps of $\Gamma_{0}(D)$. Hence $\varphi_{D} \in M_{2}\left(\Gamma_{0}(D), \chi_{D}\right)$.

\section{Chapter 3: Modular Forms with Intersection Numbers as Fourier Coefficients}

\subsection{Modular Forms of Nebentypus and the Homology of the Hilbert Modular Surface}

In Chapter 3 we return to our Hilbert modular surface $X=\mathfrak{G}^{2} / S L_{2}(\mathcal{O})$, again supposing that the discriminant of the quadratic field $K$ is a prime $p \equiv 1(\bmod 4)$. The middle homology group $H_{2}(\tilde{X})$ (all homology and cohomology is with coefficients in $\mathbb{Q}$ unless otherwise stated) of the compactification $\tilde{X}=X \cup \cup S_{k}$ of $X$ is the direct sum of $\operatorname{Im}\left(H_{2}(X) \rightarrow H_{2}(\tilde{X})\right)$ and the subspace generated by the homology classes of the curves $S_{k}$, the two subspaces being orthogonal complements of one another with respect to the intersection form. In the first component lie the homology classes $T_{N}^{c}(N=1,2, \ldots)$ defined in 1.4 and one further important class which we now describe.

On $\mathfrak{G} \times \mathfrak{G}$ we have two differential forms $\omega_{j}=-\frac{1}{2 \pi} y_{j}^{-2} d x_{j} \wedge d y_{j}(j=1,2)$, where $z_{j}=x_{j}+i y_{j}(j=1,2)$ are the coordinates. Each $\omega_{j}$ is an $S L_{2}(\mathbb{R})$-invariant form on $\mathfrak{H}$, so $\omega_{1}$ and $\omega_{2}$ are $S L_{2}(\mathcal{O})$-invariant; they can therefore be considered as differential forms on the smooth non-compact surface $X^{\prime}$ obtained from $X$ by removing the finitely many singular points (quotient singularities). The sum $\omega_{1}+\omega_{2}$ is the first Chern form $c_{1}$ on $X^{\prime}$, while the product $\omega=\omega_{1} \wedge \omega_{2}$ is the second Chern form (Gauss-Bonnet form) $c_{2}$; clearly $c_{1} \wedge c_{1}=2 c_{2}$. Then ([4], 1.3 , Eq. (9))

$$
\int_{X^{\prime}} c_{1} \wedge c_{1}=2 \int_{X^{\prime}} \omega=4 \zeta_{K}(-1),
$$

where $\zeta_{K}(s)(s \in \mathbb{C})$ denotes the Dedekind zeta-function of $K$. On the other hand, in ([4], p. 229) it is shown how the forms $\omega_{j}$ can be modified by coboundaries to obtain differential forms with compact support in $X^{\prime}$; extending these forms to $\tilde{X} \supset X^{\prime}$ by 0 on the complement of $X^{\prime}$, we obtain differential forms on $\tilde{X}$ representing cohomology classes in $\operatorname{Im}\left(H_{c}^{2}\left(X^{\prime}\right) \rightarrow H^{2}(\tilde{X})\right.$ ), or equivalently (by 
Poincaré duality), in $\operatorname{Im}\left(H_{2}\left(X^{\prime}\right) \rightarrow H_{2}(\tilde{X})\right)$. We denote the homology class corresponding to $\frac{1}{4}\left(\omega_{1}+\omega_{2}\right)$ by $T_{0}^{c}$. This seemingly strange notation is easily justified: the restriction of $\omega_{1}$ or $\omega_{2}$ to any $T_{N}$ is just the standard volume form, so by (46) of 1.5

$$
T_{0}^{c} T_{N}=\frac{1}{2} \operatorname{vol}\left(T_{N}\right)=-\frac{1}{24} \sum_{d \mid N}\left(\chi_{p}(d)+\chi_{p}(N / d)\right) d \quad(N>0),
$$

whereas in Theorem 4 of Chapter 1 we proved that

$$
\begin{array}{r}
T_{M}^{c} T_{N}=\frac{1}{2} \sum_{d \mid(M, N)}\left(\chi_{p}(d)+\chi_{p}(N / d)\right) d\left(H_{p}\left(\frac{M N}{d^{2}}\right)+I_{p}\left(\frac{M N}{d^{2}}\right)\right) \\
\left(M, N>0, v_{p}(N) \leqq v_{p}(M)\right) .
\end{array}
$$

Since $H_{p}(0)=-\frac{1}{12}$ and $I_{p}(0)=0$, Equation (2) is formally the special case $M=0$ of Equation (3). By (1), we have the formula

$$
T_{0}^{c} T_{0}^{c}=\frac{1}{4} \zeta_{K}(-1)
$$

for the self-intersection number of $T_{0}^{c}$. We can now state the main result of this paper.

Theorem 1. For each homology class $K$ in the subspace of $\mathrm{H}_{2}(\tilde{X})$ generated by the $T_{N}^{c}(N \in \mathbb{N})$, the function

$$
\Phi_{K}(z)=\sum_{N=0}^{\infty} T_{N}^{c} K e^{2 \pi i N z} \quad(z \in \mathfrak{H}),
$$

given by a Fourier expansion whose $N$-th coefficient is the intersection number of $K$ with $T_{N}^{c}$, is a modular form of weight 2 , level $p$ and Nebentypus $\chi_{p}$. It is an Eisenstein series for $K=T_{0}^{c}$ and a cusp form for $K$ orthogonal to $T_{0}^{c}$. The map $K \mapsto \Phi_{K}$ from the space generated by the $T_{N}^{c}$ to the space $M_{2}\left(\Gamma_{0}(p), \chi_{p}\right)$ is injective.

Proof ${ }^{2}$. We begin by recalling the structure of $M_{2}\left(\Gamma_{0}(p), \chi_{p}\right)$. The group $\Gamma_{0}(p)$ has two cusps, $\infty$ and 0 , and correspondingly two Eisenstein series $E_{1}$ and $E_{2}$, and $M_{2}\left(\Gamma_{0}(p), \chi_{p}\right)$ is the direct sum of the space of cusp forms $S_{2}\left(\Gamma_{0}(p), \chi_{p}\right)$ and the two-dimensional space generated by $E_{1}$ and $E_{2}$. The Eisenstein series have the Fourier expansions

$$
\begin{aligned}
& E_{1}(z)=\sum_{N=1}^{\infty}\left(\sum_{d \mid N} \chi_{p}(N / d) d\right) q^{N}, \\
& E_{2}(z)=-\frac{p^{3 / 2}}{4 \pi^{2}} L\left(2, \chi_{p}\right)+\sum_{N=1}^{\infty}\left(\sum_{d \mid N} \chi_{p}(d) d\right) q^{N}
\end{aligned}
$$

([28], p. 818, Satz 12), where as usual $q=e^{2 \pi i z}$. The Hecke operators $T(1)=$ id, $T(2), \ldots$ act on $M_{2}\left(\Gamma_{0}(p), \chi_{p}\right)$ as follows: if $f=\sum_{n=0}^{\infty} a(n) q^{n}$ is in $M_{2}\left(\Gamma_{0}(p), \chi_{p}\right)$, then

$$
f \mid T(m)=\sum_{n=0}^{\infty}\left(\sum_{d \mid(n, m)} \chi_{p}(d) d a\left(m n / d^{2}\right)\right) q^{n} .
$$

2 Eichler (in a letter of May 7, 1974) has proposed a different possible method of showing that $\Phi_{K}$ is a modular form, based on Siegel's work on indefinite quadratic forms and not requiring explicit knowledge of the intersection numbers, but a proof along these lines has not yet been given 
The Eisenstein series $E_{1}$ and $E_{2}$ are eigenfunctions of all $T(m)$, the eigenvalue being in each case simply the $m$-th Fourier coefficient. The space $S_{2}\left(\Gamma_{0}(p), \chi_{p}\right)$ has a basis of normalized Hecke eigenfunctions. Each such eigenfunction has a Fourier expansion $f(z)=\sum_{n=1}^{\infty} a(n) q^{n}$ with $a(1)=1$ and $f \mid T(n)=a(n) f$ for all $n$, so Equation (6) implies the identity

$$
a(n) a(m)=\sum_{d \mid(m, n)} \chi_{p}(d) d a\left(m n / d^{2}\right) \quad(m, n>0)
$$

for the Fourier coefficients of $f$. In particular,

$$
a\left(p^{v} n\right)=a(p)^{v} a(n)
$$

for all $n>0, v \geqq 0$. Finally, the coefficients $a(n)$ of a normalized eigenfunction satisfy

$$
\overline{a(n)}=\chi_{p}(n) a(n) \quad \text { if } p \nmid n
$$

(i.e. the $n$-th coefficient is real if $\left(\frac{n}{p}\right)=1$ and pure imaginary if $\left.\left(\frac{n}{p}\right)=-1\right)$ and $|a(p)|^{2}=p$

([27], p. 785). Therefore, if $f(z)=\sum a(n) q^{n}$ is a normalized eigenfunction, $\overline{f(-\bar{z})}=\sum \overline{a(n)} q^{n}$ the normalized eigenfunction with eigenvalues $\overline{a(n)}$, the function

$$
f_{+}(z)=\frac{1}{2}(f(z)+\overline{f(-\bar{z})})=\sum \operatorname{Re}(a(n)) q^{n}
$$

is a modular form having the property that its $n$-th Fourier coefficient vanishes for all $n$ with $\left(\frac{n}{p}\right)=-1$. Denote the space of all modular forms with this property by $M_{2}^{+}\left(\Gamma_{0}(p), \chi_{p}\right)$ and its intersection with the space of cusp forms by $S_{2}^{+}\left(\Gamma_{0}(p), \chi_{p}\right)$. Then the map $f \mapsto f_{+}$extends by linearity to a projection map

$$
\pi_{+}: S_{2}\left(\Gamma_{0}(p), \chi_{p}\right) \rightarrow S_{2}^{+}\left(\Gamma_{0}(p), \chi_{p}\right)
$$

and similarly the map

$$
f(z) \mapsto f_{-}(z)=\frac{1}{2}(f(z)-\overline{f(\bar{z})})=i \sum \operatorname{Im}(a(n)) q^{n}
$$

extends to a projection $\pi_{-}$from $S_{2}\left(\Gamma_{0}(p), \chi_{p}\right)$ to the space $S_{2}^{-}\left(\Gamma_{0}(p), \chi_{p}\right)$ of cusp forms whose $n$-th Fourier coefficient is 0 whenever $\left(\frac{n}{p}\right)=1$. Clearly $\pi_{+}+\pi_{-}=$id. The spaces $S_{2}^{+}\left(\Gamma_{0}(p), \chi_{p}\right)$ and $S_{2}^{-}\left(\Gamma_{0}(p), \chi_{p}\right)$ are disjoint, since a modular form in their intersection would have the form $\sum c_{n} q^{p n}$ and hence vanish by virtue of a lemma of Hecke (cf. Ogg [31], Lemma 6, p. 32). Therefore $S_{2}\left(\Gamma_{0}(p), \chi_{p}\right)$ is the direct sum of $S_{2}^{+}\left(\Gamma_{0}(p), \chi_{p}\right)$ and $S_{2}^{-}\left(\Gamma_{0}(p), \chi_{p}\right)$, both summands having the same dimension. From (5) we see that $E_{1} \pm E_{2} \in M_{2}^{ \pm} \cdot\left(\Gamma_{0}(p), \chi_{p}\right)$, so the same statements apply to the whole space $M_{2}\left(\Gamma_{0}(p), \chi_{p}\right)$, with $\pi_{ \pm}$extended to this space by

$$
\pi_{ \pm}\left(E_{1}\right)= \pm \pi_{ \pm}\left(E_{2}\right)=\frac{1}{2}\left(E_{1} \pm E_{2}\right) \text {. }
$$


In particular,

$$
\operatorname{dim} M_{2}^{+}\left(\Gamma_{0}(p), \chi_{p}\right)=\operatorname{dim} S_{2}^{+}\left(\Gamma_{0}(p), \chi_{p}\right)+1 .
$$

We can now prove Theorem 1. For the first assertion it suffices to consider $K=T_{M}^{c}(M \in \mathbb{N})$. By (2) and (4) we have

$$
\Phi_{T_{0}^{c}}(z)=\frac{1}{4} \zeta_{K}(-1)-\frac{1}{24} \sum_{N=1}^{\infty} \sum_{d \mid N}\left(\chi_{p}(d)+\chi_{p}(N / d)\right) d q^{N} .
$$

By the functional equation of $\zeta_{K}(s)$ and the decomposition $\zeta_{K}(s)=\zeta(s) L\left(s, \chi_{p}\right)$ we have

$$
\frac{1}{4} \zeta_{K}(-1)=\frac{p^{3 / 2}}{16 \pi^{4}} \zeta_{K}(2)=\frac{p^{3 / 2}}{96 \pi^{2}} L\left(2, \chi_{p}\right)
$$

and comparing with (5) we find that

$$
\Phi_{T_{0}^{c}}=-\frac{1}{24}\left(E_{1}+E_{2}\right) \text {. }
$$

For $M>0$, we claim that

$$
\Phi_{T_{M}^{c}}=\pi_{+}\left(\varphi_{p} \mid T(M)\right)
$$

where

$$
\varphi_{p}(z)=\sum_{N=0}^{\infty}\left(H_{p}(N)+I_{p}(N)\right) q^{N} \in M_{2}^{+}\left(\Gamma_{0}(p), \chi_{p}\right)
$$

is the modular form constructed in Chapter 2. If $\left(\frac{M}{p}\right)=1$, then the Hecke operator $T(M)$ maps $M_{2}^{+}\left(\Gamma_{0}(p), \chi_{p}\right)$ to itself, so (13) reduces to $\Phi_{T_{M}^{c}}=\varphi_{p} \mid T(M)$, which is an immediate consequence of (2), (3), (14), (6) and the definition of $\Phi_{K}$. If $\left(\frac{M}{p}\right)=-1$, then $T(M)$ interchanges $M_{2}^{+}\left(\Gamma_{0}(p), \chi_{p}\right)$ and $M_{2}^{-}\left(\Gamma_{0}(p), \chi_{p}\right)$, so the righthand side of (13) is zero, in accordance with the fact that the curve $T_{M}$ is empty in this case. Finally, if $p \mid M$ then (13) follows from (2), (3), (14) and the following lemma.

\section{Lemma 1. Let}

$$
g(z)=\sum_{n=0}^{\infty} c(n) q^{n} \in M_{2}^{+}\left(\Gamma_{0}(p), \chi_{p}\right), \quad m>0 .
$$

Then

$$
\pi_{+}(g(z) \mid T(m))=\sum_{n=0}^{\infty} c_{+}(m, n) q^{n},
$$

where $c_{+}(m, n)$ is determined by $c_{+}(m, n)=c_{+}(n, m)$ and

$$
c_{+}(m, n)=\frac{1}{2} \sum_{d \mid(m, n)}\left(\chi_{p}(d)+\chi_{p}(n / d)\right) d c\left(n m / d^{2}\right) \quad\left(v_{p}(n) \leqq v_{p}(m)\right) .
$$

Proof. It suffices to treat the two cases $g(z)=\frac{1}{2}\left(E_{1}(z)+E_{2}(z)\right), g(z)=f_{+}(z)(f$ a normalized Hecke eigenfunction), since these functions generate $M_{2}^{+}\left(\Gamma_{0}(p), \chi_{p}\right)$. 
For $g=f_{+}, f=\sum a(n) q^{n}$, we have

$$
\begin{aligned}
\pi_{+}(g(z) \mid T(m)) & =\pi_{+}\left(\frac{1}{2}(f(z)+\overline{f(-\bar{z})}) \mid T(m)\right) \\
& =\pi_{+}\left(\frac{1}{2} a(m) f(z)+\frac{1}{2} \overline{a(m)} \overline{f(-\bar{z})}\right) \\
& =\frac{1}{4}(a(m)+\overline{a(m)})(f(z)+\overline{f(-\bar{z})}),
\end{aligned}
$$

so we must show that

$$
(a(m)+\overline{a(m)})(a(n)+\overline{a(n)})=\sum_{d \mid(m, n)}\left(\chi_{p}(d)+\chi_{p}(n / d)\right) d\left(a\left(\frac{m n}{d^{2}}\right)+\overline{a\left(\frac{m n}{d^{2}}\right)}\right)
$$

if $v_{p}(n) \leqq v_{p}(m)$. Write $n=p^{v} n_{0}, m=p^{v} m_{0}$ with $p \nmid n_{0}$. Then

$$
\begin{array}{rlrl}
\sum_{d \mid(m, n)} \chi_{p}(n / d) d a\left(m n / d^{2}\right) & =\sum_{d_{0} \mid\left(m_{0}, n_{0}\right)} \chi_{p}\left(n_{0} / d_{0}\right) \cdot d_{0} p^{v} \cdot a\left(m_{0} n_{0} / d_{0}^{2}\right) \\
& =p^{v} \chi_{p}\left(n_{0}\right) \sum_{d_{0} \mid\left(m_{0}, n_{0}\right)} \chi_{p}\left(d_{0}\right) d_{0} a\left(m_{0} n_{0} / d_{0}^{2}\right) \\
& =p^{v} \chi_{p}\left(n_{0}\right) a\left(m_{0}\right) a\left(n_{0}\right) & & (\text { by }(7)) \\
& =a(p)^{v} \overline{a(p)^{v}} a\left(m_{0}\right) \overline{a\left(n_{0}\right)} & & (\text { by }(9),(10)) \\
& =a(m) \overline{a(n)} & & \text { (by (8)). }
\end{array}
$$

Adding this equation to (7) and adding the equation obtained to its complex conjugate, we obtain (15). The proof of Lemma 1 in the case of Eisenstein series is similar and will be left to the reader.

We have thus proved the first statement of Theorem 1. The second is easy: Equation (12) shows that $\Phi_{T_{0}^{c}}$ is an Eisenstein series, and $\Phi_{K}$ for a cycle $K$ with $T_{0}^{c} K=0$ is a cusp form because, by (11), any form in $M_{2}^{+}\left(\Gamma_{0}(p), \chi_{p}\right)$ with zero constant term is a cusp form. Finally, the injectivity of $K \rightarrow \Phi_{K}$ follows from the Hodge index theorem, which says that for any compact algebraic surface $Y$ the restriction of the intersection form to the subspace of $H_{2}(Y)$ generated by algebraic cycles has signature $(1, n-1)$ for some $n$ (i.e. it is non-degenerate and a diagonalized version of the intersection matrix has one positive entry and otherwise only negative entries on the diagonal). Applying this to $\tilde{X}$, we see that, since $\left(T_{0}^{c}\right)^{2}>0$ by (4), the intersection form restricted to the space of algebraic cycles orthogonal to $T_{0}^{c}$ is negative definite. The classes $T_{N}^{c}$ are all algebraic. If $K$ is in the space they generate and $T_{N}^{c} K=0$ for all $N \geqq 0$, then in particular $T_{0}^{c} K=0$ and $K K=0$, so $K=0$. This completes the proof of Theorem 1 .

Theorem 1 was stated as a conjecture in [5]. In view of our new information we can improve some of the discussion given in [5]. Let us write $\mathbf{H}$ for $H^{2}(\tilde{X} ; \mathbb{C})$, $\mathbf{F}$ for the subspace of $\mathbf{H}$ generated by the Poincaré duals of the homology classes $T_{N}^{c}(N=0,1,2, \ldots), \mathbf{M}$ for the space $M_{2}^{+}\left(\Gamma_{0}(p), \chi_{p}\right)$ (the letters are meant to suggest "homology", "curves $F_{N}$ " and "modular forms", respectively). The content of Theorem 1 is the existence of an injective map

$\Phi: \mathbf{F} \rightarrow \mathbf{M}, \quad K \mapsto \sum T_{N}^{c} K q^{N}$.

In the proof of the injectivity we showed that a class $K \in \mathbf{F}$ which is orthogonal to all of the $T_{N}^{c}$ is zero, i.e. the space $\mathbf{F}$ is disjoint from its orthogonal complement $\mathbf{F}^{\perp}$ in $\mathbf{H}$. Hence $\mathbf{H}=\mathbf{F} \oplus \mathbf{F}^{\perp}$. It follows immediately that $\Phi_{\alpha}(z)=\sum \alpha\left[T_{N}^{c}\right] e^{2 \pi i N z}$ 
belongs to $\mathbf{M}$ for any cohomology class $\alpha \in \mathbf{H}$, i.e. the map $\Phi$ extends to a map $\Phi: \mathbf{H} \rightarrow \mathbf{M}$. This map is injective on $\mathbf{F}$ and zero on $\mathbf{F}^{\perp}$, so we have $\operatorname{dim}(\operatorname{Im} \Phi)=\operatorname{dim} \mathbf{F}$. We now give a conjectural description of the subspace $\mathbf{F}$ of $\mathbf{H}$ in purely homological terms: we will define a certain subspace $\mathbf{U}$ of $\mathbf{H}$ which contains all the $T_{N}^{c}$ and conjecture that this "upper bound" $\mathbf{U}$ for $\mathbf{F}$ in fact coincides with $\mathbf{F}$.

The Poincare duals of the classes $T_{N}^{c}$ have the following properties:

(i) They are of type $(1,1)$ in the Hodge decomposition of $H^{2}(\tilde{X} ; \mathbb{C})$;

(ii) They are invariant under the involution $\tau$ of $\tilde{X}$ induced by the involution $\left(z_{1}, z_{2}\right) \mapsto\left(z_{2}, z_{1}\right)$ of $\mathfrak{H}^{2}$

(iii) They are orthogonal to the curves $S_{j}$ of the resolution of the cusp, i.e. they lie in $\operatorname{Im}\left(H_{c}^{2}(X ; \mathbb{C}) \rightarrow H^{2}(\tilde{X} ; \mathbb{C})\right)$;

(iv) They are in the kernel of $t_{n}^{*}-t_{n^{\prime}}^{*}$ for all ideals $n \subset \mathcal{O}$, where $t_{n}$ is the Hecke correspondence on $X$.

We take for $\mathbf{U}$ the space of all cohomology classes satisfying (i)-(iv). Since $H^{*}(X / \tau) \cong H^{*}(X)^{\tau}$, one can identify the (bigger) space of all cohomology classes satisfying (i)-(iii) with the subspace

$$
\mathbb{S}^{1,1}=\operatorname{Im}\left(H_{c}^{2}(X / \tau ; \mathbb{C}) \rightarrow H^{2}(V ; \mathbb{C})\right) \cap H^{1,1}(V)
$$

of $H^{2}(V)$, where $V$ is the minimal resolution of $\bar{X} / \tau(\bar{X}=X \cup\{$ cusps $\})$. Thus $\mathbf{U}$ can be considered as a subspace of $\mathfrak{\Xi}^{1,1}$. The notations $\mathbf{U}, V, \mathfrak{\Xi}^{1,1}$ are the same as in [5], where it was deduced from the formulas for the arithmetic genera of $\bar{X}$ and $V$ that

$$
\operatorname{dim} \mathbf{U}=\left[\frac{p-5}{24}\right]+1
$$

On the other hand, Hecke [28] has shown that the dimension of $\mathbf{M}=M_{2}^{+}\left(\Gamma_{0}(p), \chi_{p}\right)$ is also given by $\left[\frac{p-5}{24}\right]+1$. (As mentioned in the Introduction, it was this coincidence of dimensions which formed the original motivation for the present investigation.) Summarizing, we have $\mathbf{F} \subset \mathbf{U} \subset \mathbf{H}$ and a map $\Phi: \mathbf{H} \rightarrow \mathbf{M}$ with $\operatorname{dim}(\operatorname{Im} \Phi)=\operatorname{dim} \mathbf{F}$ and $\operatorname{dim} \mathbf{U}=\operatorname{dim} \mathbf{M}$. The only reasonable way to explain this is to assume the truth of the following conjecture:

Conjecture 1. We have $\mathbf{F}=\mathbf{U}$ and the map $\Phi: \mathbf{U} \rightarrow \mathbf{M}$ is an isomorphism.

By Theorem 1, it would suffice to show that $\Phi: \mathbf{F} \rightarrow \mathbf{M}$ is surjective, and by (13) this can be expressed purely in terms of modular forms, with no reference to the Hilbert modular surface:

Conjecture 1'. The modular forms $\pi_{+}\left(\varphi_{p} \mid T(M)\right)$ span $M_{2}^{+}\left(\Gamma_{0}(p), \chi_{p}\right)$.

\subsection{The Relationship to the Doi-Naganuma Mapping}

Let $\subseteq=S_{2}\left(S L_{2}(\mathcal{O})\right)$ be the space of cusp forms for the Hilbert modular group. To each such cusp form $F\left(z_{1}, z_{2}\right)$ we associate the differential form

$$
\frac{i}{2}\left(F\left(\varepsilon_{0} z_{1}, \varepsilon_{0}^{\prime} \bar{z}_{2}\right) d z_{1} \wedge d \bar{z}_{2}+F\left(\varepsilon_{0} z_{2}, \varepsilon_{0}^{\prime} \bar{z}_{1}\right) d z_{2} \wedge d \bar{z}_{1}\right)
$$


on $X^{\prime}=X-$ \{quotient singularities $\}$, where $\varepsilon_{0}>0>\varepsilon_{0}^{\prime}$ is a fundamental unit for $K$. This form is cohomologous to a form with compact support and thus leads to a cohomology class on $\tilde{X}$ satisfying the properties (i), (ii), (iii) listed above. This

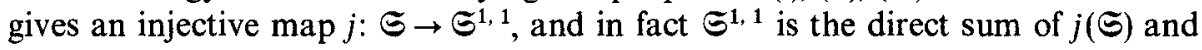
the one-dimensional space generated by the Poincare dual of $T_{0}^{c}$. Since this latter class comes from the differential form

$$
\frac{1}{16 \pi i}\left(y_{1}^{-2} d z_{1} \wedge d \bar{z}_{1}+y_{2}^{-2} d z_{2} \wedge d \bar{z}_{2}\right)
$$

whose exterior product with the form (16) vanishes identically, we see that $j(\Xi)$ is orthogonal to $T_{0}^{c}$ and hence by Theorem 1 the image of $j(\Theta)$ under $\Phi$ is contained in the space of cusp forms. In this way the map $\Phi$ constructed in 3.1 leads to a map

$$
\Phi \circ j: \Im=S_{2}\left(S L_{2}(\mathcal{O})\right) \rightarrow S_{2}^{+}\left(\Gamma_{0}(p), \chi_{p}\right)
$$

involving only cusp forms, and it is natural to ask for a description of this map purely in terms of the theory of modular forms.

A non-degenerate scalar product (Petersson product) is defined on $S_{2}\left(\Gamma_{0}(p), \chi_{p}\right)$ by

$$
(f, g)=\int_{\mathfrak{S} / \Gamma_{0}(p)} f(z) \overline{g(z)} d x d y \quad(z=x+i y),
$$

and similarly on $\subseteq$ by

$$
(F, G)=\int_{\mathfrak{S}^{2} / \mathbf{S L}_{2}(\mathcal{C})} F\left(z_{1}, z_{2}\right) \overline{G\left(z_{1}, z_{2}\right)} d x_{1} d y_{1} d x_{2} d y_{2} .
$$

The Petersson product on $\subseteq$ corresponds under $j$ roughly to the cup product pairing in chomology; more precisely, for $F, G \in \Xi$,

$$
(j(F) \cup j(G))[\tilde{X}]=-2(F, \tilde{G}),
$$

where $\tilde{G}$ is the cusp form defined by $\tilde{G}\left(z_{1}, z_{2}\right)=\overline{G\left(-\bar{z}_{2},-\bar{z}_{1}\right)}$.

In [37], Doi and Naganuma defined a method of "lifting" cusp forms in one variable (for $S L_{2}(\mathbb{Z})$ ) to Hilbert cusp forms for $S L_{2}(\mathcal{O})$, and later Naganuma [38] carried over the construction to the case of forms of Nebentypus, constructing for each even $k \geqq 2$ a map

$$
\imath: S_{k}\left(\Gamma_{0}(p), \chi_{p}\right) \rightarrow S_{k}\left(S L_{2}(\mathcal{O})\right)
$$

(in fact, Naganuma assumes that $k>2$ and that the class number of $K$ is 1 , but these restrictions can be lifted). This map sends Hecke eigenfunctions to Hecke eigenfunctions, it factors through the projection $\pi_{+}: S_{k}\left(\Gamma_{0}(p), \chi_{p}\right) \rightarrow S_{k}^{+}\left(\Gamma_{0}(p), \chi_{p}\right)$, and its image lies in the subspace $\bigcap_{n} \operatorname{Ker}\left(T(n)-T\left(n^{\prime}\right)\right)$ of $\Xi$, where $n$ runs through the ideals of $\mathcal{O}$ and the $T(n)$ are Hecke operators in $\subseteq$ (this subspace is just $j^{-1} \mathbf{U}$, with $\mathbf{U}$ as in 3.1). The purpose of this section is to motivate the following conjecture.

Conjecture 2. The map $\Phi \circ j$ form $S_{2}\left(S L_{2}(\mathcal{O})\right)$ to $S_{2}\left(\Gamma_{0}(p), \chi_{p}\right)$ is, up to a factor, the adjoint of 1 with respect to the Petersson product. 
Notice that this conjecture would imply Conjecture 1 , since $\imath$ is known to be injective on $S_{2}^{+}\left(\Gamma_{0}(p), \chi_{p}\right)$.

In [40] a series of cusp forms $\omega_{1}^{(k)}, \omega_{2}^{(k)}, \ldots \in S_{k}\left(S L_{2}(\mathcal{O})\right)$ was constructed for any even $k \geqq 2$, and it was shown that the function

$$
\Omega\left(z_{1}, z_{2} ; z\right)=\sum_{N=1}^{\infty} N^{k-1} \omega_{N}^{(k)}\left(z_{1}, z_{2}\right) e^{2 \pi i N z} \quad\left(z_{1}, z_{2}, z \in \mathfrak{H}\right)
$$

considered as a function of $z$, belongs to $S_{k}\left(\Gamma_{0}(p), \chi_{p}\right)$ and that this function of three variables is the "kernel" of the Doi-Naganuma mapping in the sense that

$$
l(f)\left(z_{1}, z_{2}\right)=\frac{1}{C_{k}} \int_{\mathfrak{S} / \Gamma_{0}(p)} \Omega\left(z_{1}, z_{2} ;-\bar{z}\right) f(z) y^{k-2} d x d y
$$

(with $C_{k}=8 \pi(2 i)^{-k} /(k-1)$ ) for all $f \in S_{k}\left(\Gamma_{0}(p), \chi_{p}\right)$. The definition of $\omega_{N}^{(k)}$ if $k>2$ is

$$
\omega_{N}^{(k)}\left(z_{1}, z_{2}\right)=p^{k / 2} \sum_{a, b, \lambda}\left(a \sqrt{p} z_{1} z_{2}+\lambda z_{2}-\lambda^{\prime} z_{1}+b \sqrt{p}\right)^{-k},
$$

where the summation runs over all $a, b \in \mathbb{Z}, \lambda \in \mathcal{O}$ satisfying $\lambda \lambda^{\prime}+a b p=-N$ (for $k=2$ this series diverges and the definition is more complicated); the similarity with the definition of the curve $T_{N}$ springs at once to the eye. Now the fact that $t$ is given by the Petersson product in $z$ with $C_{k}^{-1} \Omega\left(-\bar{z}_{1},-\bar{z}_{2} ; z\right)$ is equivalent to the statement that the adjoint map $\imath^{*}$ is given by the Petersson product in $\left(z_{1}, z_{2}\right)$ with the complex conjugate of the same function, i.e. that

$$
l^{*}(F)(z)=C_{k}^{-1} \sum_{N=1}^{\infty} N^{k-1}\left(F, \omega_{N}^{(k)}\right) e^{2 \pi i N z}
$$

for any $F \in S_{k}\left(S L_{2}(\mathcal{O})\right.$ ). For $k=2$ this says (using (17) and the fact that $\tilde{\omega}_{N}^{(2)}=\omega_{N}^{(2)}$ )

$$
t^{*}(F)(z)=-\frac{1}{4 \pi} \sum_{N=1}^{\infty} N\left(j\left(\omega_{N}^{(2)}\right) \cup j(F)\right)[\tilde{X}] e^{2 \pi i N z},
$$

whereas by definition

$$
\Phi \circ j(F)(z)=\sum_{N=1}^{\infty} j(F)\left[T_{N}^{c}\right] e^{2 \pi i N z} .
$$

Therefore Conjecture 2 is equivalent to

Conjecture 2'. The images under $j$ of the Hilbert cusp forms $N \omega_{N}^{(2)}\left(z_{1}, z_{2}\right)$ $(N=1,2, \ldots)$ are, up to a constant factor, the Poincare duals of the homology classes $T_{N}^{c}$.

In particular, this would imply that

$$
\left.H_{p}(N)+I_{p}(N)=\text { (const. }\right) \cdot N \cdot \int_{\mathfrak{S} / S L_{2}(\mathbf{z})} \omega_{N}^{(2)}\left(\varepsilon_{0} z, \varepsilon_{0}^{\prime} \bar{z}\right) d x d y,
$$

since the expression on the left equals $T_{N}^{c} T_{1}$ and the integral on the right equals $\frac{1}{2} j\left(\omega_{N}^{(2)}\right)\left[T_{1}\right]$. The justification for making Conjectures 2 and $2^{\prime}$ is that the 
corresponding equation for the forms of higher weight is true: if $k>2$, then

$$
c_{k}(N)=\text { (const.) } \cdot N^{k-1} \cdot \int_{\mathfrak{S} / S L_{2}(\mathbb{z})} \omega_{N}^{(k)}\left(\varepsilon_{0} z, \varepsilon_{0}^{\prime} \bar{z}\right) y^{k-2} d x d y,
$$

where $c_{k}(N)$ is the arithmetical function defined in 2.1, with $c_{2}(N)=H_{p}(N)+I_{p}(N)$. This will be proved in [36]. Unfortunately, the proof breaks down for $k=2$ because some of the sums and integrals involved no longer converge absolutely. Note that (18) together with the fact that $\Omega\left(z_{1}, z_{2} ; z\right)$ is a modular form in $z$ immediately imply the result that $\varphi_{p, k}(z)=\sum c_{k}(N) e^{2 \pi i N z}$ is a modular form, so a proof of (18) in the case $k=2$ would give another proof of the main result of Chapter 2.

We mention one other result for higher weights, also to be proved in [36], which if true for $k=2$ would imply Conjecture 1 ', namely the following characterization of the function $\varphi_{p, k}$ in terms of the Petersson product:

Let $k>2$. Then for $f(z)=\sum a(n) e^{2 \pi i n z} \in S_{k}^{+}\left(\Gamma_{0}(p), \chi_{p}\right)$, the Petersson product of $\varphi_{p, k}$ and $f$ is given by

$$
\left(f, \varphi_{p, k}\right)=\text { (const.) } \cdot \sum_{n=1}^{\infty} \frac{\varepsilon(n) a\left(n^{2}\right)}{n^{k}},
$$

where $\varepsilon(n)=1$ or 2 according as $p \nmid n$ or $p \mid n$.

It is not hard to deduce from this result that the functions $\pi_{+}\left(\varphi_{p, k} \mid T(M)\right)$ $(M=1,2, \ldots)$ generate $M_{k}^{+}\left(\Gamma_{0}(p), \chi_{p}\right)$; thus the analogue of Conjecture $1^{\prime}$ for higher weights is true.

\section{References}

\section{Hilbert Modular Surfaces}

1. Eichler, M.: Über die Einheiten der Divisionsalgebren. Math. Ann. 114, 635-654 (1937)

2. Hammond, W.F.: The modular groups of Hilbert and Siegel. Amer. J. of Math. 88, 497-516 (1966)

3. Herrmann, O.: Über Hilbertsche Modulfunktionen und die Dirichletschen Reihen mit Eulerscher Produktentwicklung. Math. Ann. 127, 357-400 (1954)

4. Hirzebruch, F.: Hilbert modular surfaces. L'Ens. Math. 19, 183-281 (1973)

5. Hirzebruch, F.: Kurven auf den Hilbertschen Modulflächen und Klassenzahlrelationen. Classification of algebraic varieties and compact complex manifolds. Lecture Notes in Math. 412, pp. 75-93. Berlin-Heidelberg-New York: Springer 1974

6. Hirzebruch, F., Van de Ven, A.: Hilbert modular surfaces and the classification of algebraic surfaces. Inventiones math. 23, 1-29 (1974)

7. Hirzebruch, F., Zagier, D.: Classification of Hilbert modular surfaces. To appear

8. Prestel, A.: Die elliptischen Fixpunkte der Hilbertschen Modulgruppen. Math. Ann. 177, 181-209 (1968)

\section{Number Theory, Especially Binary Quadratic Forms}

9. Borewicz, S., Safarevič, I. R.: Zahlentheorie. Basel-Stuttgart: Birkhäuser 1966

10. Butts, H.S., Pall, G.: Modules and binary quadratic forms. Acta Arithm. 15, 23-44 (1968)

11. Dirichlet, G.L.: Über eine Eigenschaft der quadratischen Formen, Ber. d. Königl. Preuss. Akad. d. Wiss. (1840). Gesammelte Werke, Bd. I, 497-502. Berlin: Reimer 1889 
12. Landau, E.: Vorlesungen über Zahlentheorie (Aus der elementaren Zahlentheorie). Leipzig: Hirzel 1927

13. Pall, G.: The structure of the number of representations function in a positive binary quadratic form. Math. Z. 36, 321-343 (1933)

14. Weber, H.: Beweis des Satzes, daß jede eigentliche primitive quadratische Form unendlich viele Primzahlen darzustellen fähig ist. Math. Ann. 20, 301-329 (1882)

15. Zagier, D.: On the values at negative integers of the zeta-function of a real quadratic field. To appear in L'Ens. Math. (1976)

\section{Class Number Relations}

The literature concerning class number relations is very extensive. We give a brief selection only (in chronological order). For further references up to 1923 see Chapter VI (written by G. Cresse) of Dickson's History of the Theory of Numbers, Vol. III, Carnegie, Washington, 1919 (reprinted by Chelsea, New York, 1971).

16. Kronecker, L.: Über quadratische Formen von negativer Determinante. Monatsber. d. Königl. Preuss. Akad. d. Wiss., Berlin (1875). Gesammelte Werke, Bd. IV, 245-259. Leipzig: Teubner 1929

17. Hurwitz, A.: Über Relationen zwischen Klassenzahlen binärer quadratischer Formen von negativer Determinante. Math. Ann. 25 (1885). Mathematische Werke, Bd. II, 8-50. Basel-Stuttgart: Birkhäuser 1963

18. Klein, F., Fricke, R.: Vorlesungen über die Theorie der elliptischen Modulformen, Bd. II, Abschnitt 4, Kap. 6 (204-236) and Abschnitt 6, Kap. 5 (637-667). Leipzig: Teubner 1892

19. Mordell, L.J.: On the generating function of the series $\sum F(n) q^{n}$ where $F(n)$ is the number of uneven classes of binary quadratics of determinant $-n$. Mess. of Math. 50, 113-128 (1920)

20. Hecke, E.: Neue Herleitung der Klassenzahlrelationen von Hurwitz und Kronecker. Nachr. d. Königl. Ges. d. Wiss. zu Göttingen, Math.-phys. Kl. (1926). Mathematische Werke, pp. 499-504. Göttingen: Vandenhoeck \& Ruprecht 1970

21. Eichler, M.: On the class number of imaginary quadratic fields and the sums of divisors of natural numbers. J. Ind. Math. Soc. 19, 153-180 (1955)

22. Mordell, L.J.: On recurrence formulas for the number of classes of definite binary quadratic forms. J. Ind. Math. Soc. 24, 367-378 (1960)

\section{Modular Forms of One Variable}

23. Cohen, $H$.: Sums involving the values at negative integers of $L$ functions of quadratic characters. Math. Ann. 217, 271-285 (1975)

24. Eichler, M.: The basis problem for modular forms and the traces of the Hecke operators. Modular Functions of One Variable I, Lecture Notes in Math. 320, pp. 75-152. Berlin-Heidelberg-New York: Springer 1973

25. Hecke, E.: Theorie der Eisensteinschen Reihen höherer Stufe und ihre Anwendung auf Funktionentheorie und Arithmetik, Abh. Math. Sem. Hamburg Univ. 5 (1927). Werke, 461-486

26. Hecke, E.: Über Modulfunktionen und die Dirichletschen Reihen mit Eulerscher Produktentwicklung. II. Math. Ann. 114 (1937). Werke, 672-707

27. Hecke, E.: Über die Darstellung der Determinante einer positiven quadratischen Form durch die Form. Vierteljahrschrift d. Naturforschenden Gesellschaft in Zürich 85(1940). Werke, 782-788

28. Hecke, E.: Analytische Arithmetik der positiven quadratischen Formen. Kgl. Danske Vid. Selskab. Math.-fys. Med. XIII, 12 (1940). Werke, 789-918

29. Li, W. W.: Newforms and functional equations. Math. Ann. 212, 285-315 (1975)

30. Ogg, A.: Modular forms and Dirichlet series. Benjamin, New York-Amsterdam (1969)

31. Ogg, A.: Survey of modular functions of one variable. Modular functions of one variable I. Lecture Notes in Math. 320, pp. 1-36. Berlin-Heidelberg-New York: Springer 1973

32. Selberg, A.: Harmonic analysis and discontinuous groups in weakly symmetric Riemannian spaces with applications to Dirichlet series. J. Ind. Math. Soc. 20, 47-87 (1956) 
33. Shimura, G.: Modular forms of half-integral weight. Modular functions of one variable I. Lecture Notes in Math. 320, pp. 57-74. Berlin-Heidelberg-New York: Springer 1973

34. Shimura, G.: Modular forms of half integral weight. Ann. of Math. 97, 440-481 (1973)

35. Zagier, D.: Nombres de classes et formes modulaires de poids $3 / 2$, C. R. Acad. Sc. Paris 281 (Sér. A), 883-886 (1975)

36. Zagier, D.: Modular forms whose Fourier coefficients involve zeta-functions of quadratic fields. To appear

The Doi-Naganuma Map

37. Doi, K., Naganuma, H.: On the functional equation of certain Dirichlet series. Inventiones math. 9, 1-14 (1969)

38. Naganuma, H.: On the coincidence of two Dirichlet series associated with cusp forms of Hecke's "Neben"-type and Hilbert modular forms over a real quadratic field. J. Math. Soc. Japan 25, 547-555 (1973)

39. Saito, H.: Algebraic extensions of number fields and automorphic forms. Kyoto Univ. Lectures in Math. 8, Tokyo: Kinokuniya 1973

40. Zagier, D.: Modular forms associated to real quadratic fields. Inventiones math. 30, 1-46 (1975)

Received March 4, 1976

F. Hirzebruch

D. Zagier

Mathematisches Institut der Universität

Wegelerstr. 10

D-5300 Bonn

Federal Republic of Germany 